\title{
Interstellar anatomy of the TeV gamma-ray peak in the IC443 supernova remnant ${ }^{\star}$
}

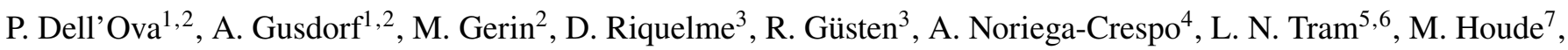 \\ P. Guillard ${ }^{8}$, A. Lehmann ${ }^{1,2}$, P. Lesaffre ${ }^{1,2}$, F. Louvet ${ }^{1,9}$, A. Marcowith ${ }^{10}$, and M. Padovani ${ }^{11}$
}

\author{
${ }^{1}$ Laboratoire de Physique de l'École Normale Supérieure, ENS, Université PSL, CNRS, Sorbonne Université, Université de Paris, \\ 75005 Paris, France \\ e-mail: pierre.dellova@ens.fr \\ 2 Observatoire de Paris, PSL University, Sorbonne Université, LERMA, 75014 Paris, France \\ ${ }^{3}$ Max-Planck-Institut für Radioastronomie (MPIfR), Auf dem Hügel 69, 53121 Bonn, Germany \\ ${ }^{4}$ Space Telescope Science Institute, 3700 San Martin Dr., Baltimore, MD 21218, USA \\ ${ }^{5}$ Stratospheric Observatory for Infrared Astronomy, Universities Space Research Association, NASA Ames Research Center, \\ MS 232-11, Moffett Field, 94035 CA, USA \\ ${ }^{6}$ University of Science and Technology of Hanoi, Vietnam Academy of Science and Technology, 18 Hoang Quoc Viet, Vietnam \\ ${ }^{7}$ Department of Physics and Astronomy, The University of Western Ontario, London, Ontario, N6A 3K7, Canada \\ ${ }^{8}$ Institut d'Astrophysique de Paris, CNRS UMR 7095, Sorbonne Université, 75014 Paris, France \\ 9 AIM, CEA, CNRS, Université Paris-Saclay, Université Paris Diderot, Sorbonne Paris Cité, 91191 Gif-sur-Yvette, France \\ ${ }^{10}$ Laboratoire Univers et Particules de Montpellier (LUPM) Université Montpellier, CNRS/IN2P3, CC72, place Eugène Bataillon, \\ 34095, Montpellier Cedex 5, France \\ ${ }^{11}$ INAF-Osservatorio Astrofisico di Arcetri, Largo E. Fermi 5, 50125 Firenze, Italy
}

Received 4 May 2020 / Accepted 13 October 2020

\begin{abstract}
Context. Supernova remnants (SNRs) represent a major feedback source from stars in the interstellar medium of galaxies. During the latest stage of supernova explosions, shock waves produced by the initial blast modify the chemistry of gas and dust, inject kinetic energy into the surroundings, and may alter star formation characteristics. Simultaneously, $\gamma$-ray emission is generated by the interaction between the ambient medium and cosmic rays (CRs), including those accelerated in the early stages of the explosion.

Aims. We study the stellar and interstellar contents of IC443, an evolved shell-type SNR at a distance of $1.9 \mathrm{kpc}$ with an estimated age of $30 \mathrm{kyr}$. We aim to measure the mass of the gas and characterize the nature of infrared point sources within the extended $\mathrm{G}$ region, which corresponds to the peak of $\gamma$-ray emission detected by VERITAS and Fermi.

Methods. We performed $10^{\prime} \times 10^{\prime}$ mapped observations of ${ }^{12} \mathrm{CO},{ }^{13} \mathrm{CO} J=1-0, J=2-1$, and $J=3-2$ pure rotational lines, as well as $\mathrm{C}^{18} \mathrm{O} J=1-0$ and $J=2-1$ obtained with the IRAM $30 \mathrm{~m}$ and APEX telescopes over the extent of the $\gamma$-ray peak to reveal the molecular structure of the region. We first compared our data with local thermodynamic equilibrium models. We estimated the optical depth of each line from the emission of the isotopologs ${ }^{13} \mathrm{CO}$ and $\mathrm{C}^{18} \mathrm{O}$. We used the population diagram and large velocity gradient assumption to measure the column density, mass, and kinetic temperature of the gas using ${ }^{12} \mathrm{CO}$ and ${ }^{13} \mathrm{CO}$ lines. We used complementary data (stars, gas, and dust at multiple wavelengths) and infrared point source catalogs to search for protostar candidates.

Results. Our observations reveal four molecular structures: a shocked molecular clump associated with emission lines extending between -31 and $16 \mathrm{~km} \mathrm{~s}^{-1}$, a quiescent, dark cloudlet associated with a line width of $\sim 2 \mathrm{~km} \mathrm{~s}^{-1}$, a narrow ring-like structure associated with a line width of $\sim 1.5 \mathrm{~km} \mathrm{~s}^{-1}$, and a shocked knot. We measured a total mass of $\sim 230, \sim 90, \sim 210$, and $\sim 4 M_{\odot}$, respectively, for the cloudlet, ring-like structure, shocked clump, and shocked knot. We measured a mass of $\sim 1100 M_{\odot}$ throughout the rest of the field of observations where an ambient cloud is detected. We found 144 protostar candidates in the region.

Conclusions. Our results emphasize how the mass associated with the ring-like structure and the cloudlet cannot be overlooked when quantifying the interaction of CRs with the dense local medium. Additionally, the presence of numerous possible protostars in the region might represent a fresh source of CRs, which must also be taken into account in the interpretation of $\gamma$-ray observations in this region.
\end{abstract}

Key words. ISM: supernova remnants - ISM: individual objects: IC443 - ISM: kinematics and dynamics - cosmic rays stars: formation

\section{Introduction}

The violent end of some stellar objects, a supernova (SN) explosion, is the beginning of an incredible sequence of energy injection in the surrounding interstellar medium (ISM). A SN

\footnotetext{
* The reduced datacubes are only available at the CDS via anonymous ftp to cdsarc.u-strasbg.fr (130.79.128.5) or via http: //cdsarc.u-strasbg.fr/viz-bin/cat/J/A+A/644/A64
}

explosion ejects material with mass ranging from $\sim 1.4$ to $\sim 20 M_{\odot}$ and a typical energy of $10^{50-52} \mathrm{erg}$ (see, e.g., Draine 2011) and drives fast shock waves about $10^{4} \mathrm{~km} \mathrm{~s}^{-1}$ ahead of the ejecta (the expelled stellar material) through the ISM. Already at early stages, SNe play a crucial, multifaceted role in the evolution of galaxies. The explosion and subsequent dispersion of matter is itself the most important source of elements heavier than nitrogen in the gas phase (François et al. 2004). The fast shocks inject 
kinetic energy that gradually decays in turbulence, which is a key process in the regulation of star formation on galactic scales (Mac Low \& Klessen 2004). Fast shock fronts are also a recognized site for the production of the bulk of cosmic rays, at least at gigaelectronvolt energies (CRs; see, e.g., Bykov et al. 2018; Tatischeff \& Gabici 2018 and references therein for recent reviews), although alternative origins are drawing more and more attention (such as superbubbles and Fermi bubbles; see, e.g., Grenier et al. 2015; Gabici et al. 2019 and references therein for recent reviews). Finally, the hot $\left(10^{6}-10^{8} \mathrm{~K}\right)$ and relatively dense $\left(1-10 \mathrm{~cm}^{-3}\right)$ conditions in the ejecta could be favorable to the synthesis of cosmic dust up to hundred years after the explosion (see recent reviews by Cherchneff 2014; Sarangi et al. 2018; Micelotta et al. 2018). After the first phases of expansion (free, then adiabatic), the temperature of the shock front drops to $10^{6} \mathrm{~K}$, allowing the gas to radiatively cool down. At this stage, the so-called supernova remnant (SNR) resembles a spherical shell of 10-20 pc radius, delimitated by regions in which shocks interact with the ambient medium.

This evolved SNR stage is also of fundamental importance to many aspects of galactic evolution. The fastest remaining shocks (between $\sim 30$ and a few hundreds of kilometers per second) dissociate and ionize the pre- and post-shock medium and generate far-ultraviolet (FUV) photons (e.g., Hollenbach \& McKee 1989). More generally, shocks heat, accelerate, and compress the ambient medium. They inject energy and trigger specific dust and gas-phase chemical processes (e.g., van Dishoeck et al. 1993), hence significantly participating in the cycle of matter in galaxies. Cosmic rays accelerated in the earlier stages of the explosion and trapped in the shock fronts now interact with the dense medium, producing observable X-ray to $\gamma$-ray photons (Gabici et al. 2009; Celli et al. 2019; Tang 2019 and references therein). Cosmic rays of Galactic origin can also be reaccelerated in the shock regions (such as in W44, e.g., Cardillo et al. 2016). Finally, evolved SNRs play a key role in star formation. Like all massive stars, the progenitor of a SN explosion forms in a cluster in a dense and inhomogeneous environment, where lower-mass stellar companions have a greater life expectancy (Montmerle 1979). During its life on the main sequence, the progenitor drives stellar winds in the surrounding medium, possibly triggering a second generation of star formation in the neighboring molecular clouds (e.g., Koo et al. 2008). The compression and cooling caused by SNR shocks might also generate star formation (e.g., Herbst \& Assousa 1977). In any case, the injection of energy exerted by SNRs in all possible forms (CRs, energetic photons, and shocks) likely alters locally the characteristics of all possible star formation events over significant spatial scales and times.

With the present study we aim to start characterizing as precisely as possible, and on fields as large as possible, the mechanisms of energy injection (shocks, photons, and CRs) exerted by an evolved SNR, and its effects on local star formation. Such a study must be performed on an evolved object, since energy injection effects can spread over the full duration of the SNR phase, and are all the more visible when the SNR is old. In particular, we want to provide support for the study of CRs properties (acceleration, composition, and diffusion) in evolved SNRs. In these objects, CRs interact with the local medium through four processes that all generate $\gamma$-ray photons: pion decay from the collision of hadronic CRs with the dense material, Bremsstrahlung from the interaction of leptonic CRs with the local dense medium, inverse Compton scattering of leptonic CRs with the local radiation field, and synchrotron emission of leptonic CRs gyrating around the local magnetic fields. Our first aim is to constrain the properties of the local medium that is the target of these interactions: the mass and density of all observed components, magnetic field structure, and radiation field structure.

Our second aim is to identify all possible sources of ongoing acceleration of "fresh" CRs additional to the "old" injection of CRs previously accelerated by the SNR. There can be two kinds of these sources: ionized regions in which kinetic energy is deposited and the magnetic field structure and ionization fraction make the acceleration possible (such as [H II] regions; see Padovani et al. 2019); or protostellar jets and outflows, where these conditions can be naturally combined. Our work can thus provide support for further studies of CR-related questions only if the study of local star formation is performed simultaneously. Indeed, Padovani et al. $(2015,2016)$ have shown that jets can accelerate low-energy CRs, which can be reaccelerated in the shock fronts of the remnant. Other studies have confirmed that supermassive star clusters neighboring SNRs can be a source of CRs (Hanabata et al. 2014). Conversely, an optimal characterization of CR action on the local formation is mandatory to better understand star formation in older galaxies. In fact, up to $z \sim 2$ the star formation efficiency is higher (Madau \& Dickinson 2014). The star formation regime observed in SNRs is reminiscent of starburst galaxies, where $\mathrm{SNe}$ from a given generation of stars affect the next one.

The threefold and intertwined goals of our study (ISM, star formation, and CR) make the study of large fields mandatory. Cosmic ray studies rely on $\gamma$-ray spectra obtained with telescopes that only provide a limited spatial resolution, typically a few arcminutes. This extent is the minimum field for which we have to characterize the ISM and star formation as best as we can. This is why we have chosen to study a $10^{\prime} \times 10^{\prime}$ field in the relatively evolved IC443 SNR (see Fig. 1). More particularly, with the present paper we investigate the physical conditions and dynamical structure of the molecular gas and its association with protostars in such a field located at the peak of $\gamma$-ray emission detected in the IC443 SNR, based on observations of the $\mathrm{CO}$ emission lines and its isotopologs. In Sect. 2 we present a summarized review of the source. In Sect. 3, we present our observations and propose a description of the morphology and kinematics of the region, emphasizing three distinct molecular components. Section 4 focuses on the measure of the gas mass for these components. First we perform a local thermodynamical equilibrium (LTE) analysis of the ${ }^{12} \mathrm{CO},{ }^{13} \mathrm{CO}$, and $\mathrm{C}^{18} \mathrm{O}$ emission lines and we build pixel-per-pixel, channel-per-channel population diagrams corrected for optical depth. Then we propose a second method using a radiative transfer code based on the large velocity gradient (LVG) approximation. In Sect. 5, we study the spectral energy distribution (SED) of point sources identified in infrared survey catalogs as well as the spatial distribution of optical point sources detected with Gaia. Finally we summarize our findings in Sect. 6.

\section{Supernova remnant IC443}

IC443 is a mixed-morphology supernova remnant, located at a distance of 1.5-2 kpc (Denoyer 1978; Welsh \& Sallmen 2003), with recent measures suggesting a kinematic distance of $1.9 \mathrm{kpc}$ (Ambrocio-Cruz et al. 2017). IC443 is an evolved SNR, yet its exact age is a matter of debate. The literature contains two kinds of value ( $\sim 3$ and $\sim 30 \mathrm{kyr})$, depending on the type of data that is analyzed. A compelling finding concerning the age and origin of the IC443 SNR was the discovery of the CXOU J061705.3+222127 pulsar wind nebula (PWN), based on Chandra X-ray observatory and Very Large Array (VLA) images 


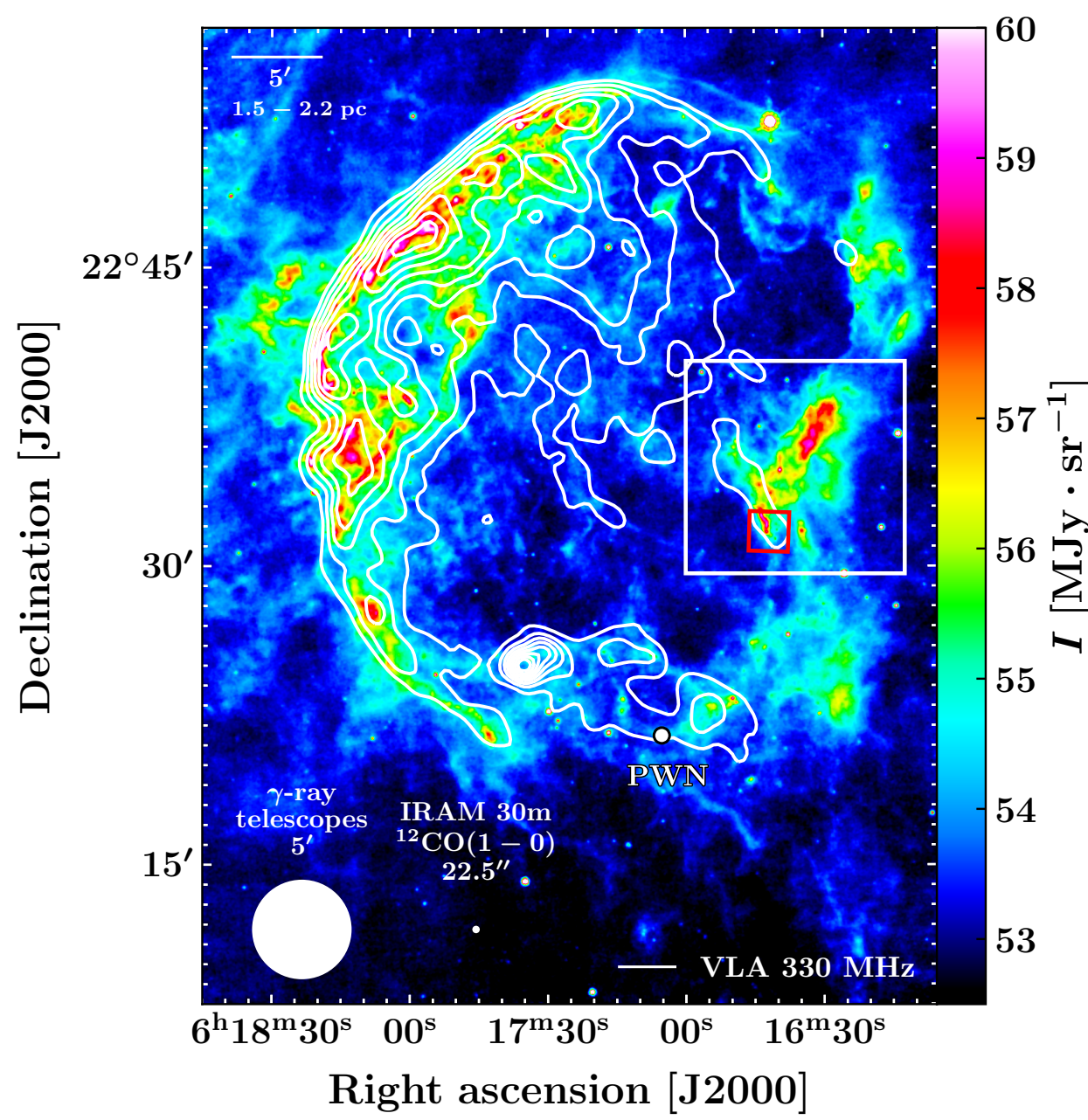

Fig. 1. Spitzer/MIPS map (colors) of IC443 at $24 \mu \mathrm{m}$ (Pinheiro Gonçalves et al. 2011; Noriega-Crespo et al. 2009). In contours, the VLA emission map shows the morphology of the synchrotron emission at $330 \mathrm{MHz}$ (Claussen et al. 1997). The white dot indicates the position of the PWN wind (Olbert et al. 2001b). The red box represents one of the fields observed by SpitzerIRS (Neufeld et al. 2004), corresponding roughly to the $\mathrm{G}$ region defined by Huang et al. (1986). The white box represents the $10^{\prime} \times 10^{\prime}$ field of our observations called "the extended G region". The IRAM 30 m instrumental beam diameter corresponding to the ${ }^{12} \mathrm{CO}(1-0)$ transition and the size of the typical PSF of $\gamma$-ray telescopes $\left(5^{\prime}\right)$ are indicated by white disks in the bottom left corner of the figure.
(Olbert et al. 2001a). The motion of the PWN is consistent with an age of $30 \mathrm{kyr}$ for the SN event, and its detection strongly supports a core-collapse formation scenario for the SNR.

IC443 displays a shell morphology in radio, with two atomic sub-shells (shells A and B, Braun \& Strom 1986). It is one of the most striking examples of a SNR interacting with neighboring molecular clouds. The most up-to-date and complete description of the structure and kinematics of both the atomic and dense molecular environment of the SNR was offered by Lee et al. $(2008,2012)$. Their $\sim 1^{\circ} \times 1^{\circ}$ map of the $J=1-0$ transition of ${ }^{12} \mathrm{CO}$ allowed us to characterize both the incomplete molecular shell interacting with shocks (toward the southern part of the SNR) and the molecular cloud that is associated with the remnant. Continuum radio emission in IC443 is partly correlated with the molecular shell and the secondary [H I] shell; see Castelletti et al. (2011) for a detailed description of the lowfrequency radio emission in IC443 at 74 and $330 \mathrm{MHz}$, and Egron et al. (2017), Loru et al. (2019) for high and very-high frequency studies at 7 and $21.4 \mathrm{GHz}$, respectively.

CO emission was observed by Denoyer (1979a) toward the SNR, revealing three shocked CO clumps along the southern molecular ridge (labeled A, B, and C). Follow-up observations of CO $J=1-0$ over a $50^{\prime} \times 50^{\prime}$ field by Huang et al. (1986) allowed the detection of new areas of shock-cloud interaction and to identify five previously unknown CO clumps, extending the classification started by Denoyer (1979a) and providing the first mention of the $\mathrm{G}$ knot. The $\mathrm{OH} 1720 \mathrm{MHz}$ line is a powerful diagnostic for the classification of SNRs interacting with molecular clouds
(Frail et al. 1996). Six masing spots were identified in IC443 by Claussen et al. (1997), all located in the $\mathrm{G}$ region delineated by Huang et al. (1986). The former authors proposed that $\mathrm{OH}$ masers spots could be promising candidates for the sites of CR acceleration. Lockett et al. (1999) improved the modeling of the shock origin for these maser lines, associated with moderate temperatures $(50-125 \mathrm{~K})$, local densities $\left(\sim 10^{5} \mathrm{~cm}^{-3}\right)$, and $\mathrm{OH}$ column densities on the order of $10^{16} \mathrm{~cm}^{-2}$, then Wardle (1999) added the effect of the dissociation of molecules by FUV photons in molecular clouds subject to CR and X-ray ionization (see Hoffman et al. 2003, Hewitt et al. 2006, 2008, 2009 for recent studies). From $J=1-0{ }^{12} \mathrm{CO}$ and $\mathrm{HCO}^{+}$emission, Dickman et al. (1992) measured a mass of $41.6 M_{\odot}$ for "clump G", and estimated that a total molecular mass of 500-2000 $M_{\odot}$ is interacting with the SNR shocks, which corresponds to 5-10\% of the SN energy considering that the average velocity of the clumps is $25 \mathrm{~km} \mathrm{~s}^{-1}$. Zhang et al. (2010) showed that two distinct structures are resolved in region $\mathrm{G}$, labeling G1 the strongest ${ }^{13} \mathrm{CO}$ peak and G2 the previously mentioned shocked clump. Lee et al. (2012) measured a mass of $57.7 \pm 0.9 M_{\odot}$ for the clump G2. Oddly, Xu et al. (2011) measured a mass of $2.06 \times 10^{3} M_{\odot}$ for "cloud G", which is much higher than the previous estimates. Several molecular shocks were mapped within the SNR using ${ }^{12}$ CO lines (e.g., White et al. 1987, Wang \& Scoville 1992 for clumps A, B, and C). In particular, the kinematics of clump $\mathrm{G}$ were characterized in detail by van Dishoeck et al. (1993), who presented observations of the rotational transition $J=3-2$ of $\mathrm{CO}$ along the shocked molecular ring at a spatial resolution of 
$20^{\prime \prime}-30^{\prime \prime}$. In the last $10 \mathrm{yr}$, the large-scale molecular contents of IC443 have been scrutinized with increasing precision and completeness, since several authors have mapped the $J=1-0$ transition of the isotopologs ${ }^{12} \mathrm{CO},{ }^{13} \mathrm{CO}$, and $\mathrm{C}^{18}$ over large fields (from $40^{\prime} \times 45^{\prime}$ to $1.5^{\circ} \times 1.5^{\circ}$; Zhang et al. 2010 , Lee et al. 2012, Su et al. 2014). Several submillimeter observations of IC443 were performed to characterize the shocked molecular gas (e.g., van Dishoeck et al. 1993 for a study of the shock chemistry in the southern ridge toward the clumps B, C, and G). Notably, the ground state of shocked ortho- $\mathrm{H}_{2} \mathrm{O}$ was detected toward the clumps B, C, and G (Snell et al. 2005).

The incomplete shell-morphology is also observed in the $J, H, K$ bands observed by 2MASS (Two-Micron All-Sky Survey; Rho et al. 2001), as well as in infrared and far-infrared observations by Spitzer-MIPS (Pinheiro Gonçalves et al. 2011; Noriega-Crespo et al. 2009) and the Wide-field Infrared Survey Explorer (WISE; Wright et al. 2010). Excitation by shocks was suggested as the most likely scenario for the emission lines detected in IR, instead of X-ray or FUV mechanisms (e.g., [O I], Burton et al. 1990). Rho et al. (2001) analyzed the emission of [O I] with shock models, suggesting a fast J-type shock $\left(\sim 100 \mathrm{~km} \mathrm{~s}^{-1}\right)$ in the NE atomic shell, and a $\mathrm{C}$ shock $\left(v_{\mathrm{s}}=\sim 30 \mathrm{~km} \mathrm{~s}^{-1}\right)$ propagating in the southern molecular ridge. In an effort to study molecular shocks, $\mathrm{H}_{2}$ pure rotational transitions were mapped toward clump G by ISOCAM (ISO; Cesarsky et al. 1999) and compared to non-stationary shock models, as well as toward clumps C and G by Spitzer-IRS (Infrared Spectrograph; Neufeld et al. 2007). Molecular clumps B, C, and G were also observed by Akari (Shinn et al. 2011) and by the Stratospheric Observatory for Infrared Astronomy (SOFIA; Reach et al. 2019). All these studies were carried out in small fields $\left(\sim 1^{\prime} \times 1^{\prime}\right)$ and allowed to put constraints on the shock velocity $\left(\sim 30 \mathrm{~km} \mathrm{~s}^{-1}\right)$ and pre-shock density $\left(\sim 10^{4} \mathrm{~cm}^{-3}\right)$, and to outline similarities with protostellar shocks in the southern ridge where we aim to focus on the extended $\mathrm{G}$ region.

The optical emission is well correlated with radio and $[\mathrm{H} \mathrm{I}]$ features, reproducing shells A and B. In particular, the SNR displays bright, filamentary structures toward the northeastern part of the remnant (Fesen \& Kirshner 1980; Alarie \& Drissen 2019). IC443 was fully mapped by the Sloan Digital Sky Survey (SDSS; York et al. 2000). There are no bright features toward the extended $\mathrm{G}$ region, but optical studies offered constraints on the global characteristics of IC443. Ambrocio-Cruz et al. (2017) estimated an age of $\sim 30 \mathrm{kyr}$ and an energy of $7.2 \times 10^{51} \mathrm{erg}$ injected in the environment by the SNR from the comparison of observations of $[\mathrm{H} \alpha]$ with SNR models (Chevalier 1974).

The shell-like structure of IC443 in radio, centrally filled in $\mathrm{X}$-rays, puts the remnant into the category of mixed-morphology SNRs (Petre et al. 1988; Rho \& Petre 1998). Observations of the hard X-ray contents of IC443 (up to $100 \mathrm{keV}$ ) by BeppoSAX show hints of shock-cloud interaction (Bocchino \& Bykov 2000). The X-ray Multi-Mirror mission (XMM-Newton) mapped IC443 in the ranges $0.3-0.5$ and $1.4-5.0 \mathrm{keV}$ with an unprecedented field of view and spatial resolution (Bocchino \& Bykov 2003), and showed that the soft X-ray emission is partly absorbed by the nearby molecular cloud (Troja et al. 2006). Troja et al. (2008) reported the detection of a ring-shaped ejecta encircling the PWN, associated with a hot metal rich plasma for which the abundances are in agreement with a core-collapse scenario.

The CR content and $\gamma$-ray emission in IC443 have been scrutinized by multiple observatories. Fermi-LAT (Large Area Telescope) detected an extended $\gamma$-ray source in the $200 \mathrm{MeV}-$ $50 \mathrm{GeV}$ energy band (Ackermann et al. 2013). Abdo et al. 2010 showed that the spectrum is well reproduced by the decay of neutral pions and pinpointed the clouds B, C, D, F, and G as targets of interaction. Tavani et al. (2010) reported the detection of $\gamma$-ray enhancement toward the NE shell in the $100 \mathrm{MeV}-$ $50 \mathrm{GeV}$ range observed by AGILE (Astro-Rivelatore Gamma a Immagini Leggero). In their hadronic model, cloud " $E$ " is the suggested target for interaction with CRs. Interestingly, the location of the $\gamma$-ray peak differs between the lower energy detections by Fermi and the Energetic Gamma Ray Experiment Telescope (EGRET, Esposito et al. 1996) and the very high energy detections by the Major Atmospheric Gamma-ray Imaging Cherenkov Telescope (MAGIC) and the Very Energetic Radiation Imaging Telescope Array System (VERITAS, Albert et al. 2007). This tendency is also verified by Tavani et al. (2010) who located the $100 \mathrm{MeV} \gamma$-ray peak close to the Fermi/EGRET position and further toward NE. Recently, VERITAS Collaboration (2015) presented an updated Fermi map where the position of the peak was shifted and consistent with VERITAS, exposing the uncertainty on the localization of the peak from the analysis of $\gamma$-ray observations. The choice of the region studied in this paper is based on the teraelectronvolt $\gamma$-ray significance map from VERITAS Collaboration (2015), which emphasizes the extended $\mathrm{G}$ region as a favorable target of high-energy $\mathrm{CR}$ interaction with dense molecular gas.

Explicit magnetic field studies toward IC443 are scarce. Wood et al. (1991) performed $6.1 \mathrm{~cm}$ polarimetric observations of the northeast rim of IC443. These authors found that the local magnetic field is rather correlated with the rim structure, but with no clear orientation (i.e., parallel or perpendicular) to it. Only Hezareh et al. (2013) conducted a polarization study toward clump G. These authors observed circular and linear polarization of the CO (1-0) and (2-1) lines with the Institut de Radioastronomie Millimétrique $30 \mathrm{~m}$ antenna (hereafter IRAM $30 \mathrm{~m}$ ), and linear polarization maps from the dust continuum with the Acatama Pathfinder EXperiment (hereafter APEX). Their study constituted a crucial step toward the characterization of the interaction of CRs with the magnetic field in the extended $\mathrm{G}$ region.

Star formation in IC443 was the focus of a few studies, but early investigations lacked sufficient data to make clear findings. Recently, Xu et al. (2011) used color criteria for the point sources detected by the Infrared Astronomical Satellite (IRAS) and 2MASS to identify protostellar objects and young stellar object (YSO) candidates and showed their association to several regions of the SNR interacting with neighboring molecular structures, including clump G. Su et al. (2014) confirmed the shell structure of the distribution of YSO candidates using a selection method based on color-color diagrams inferred from WISE bands 1,2 , and 3 and 2MASS band $K$. Both studies concluded that the formation of this YSO population is likely to have been triggered by the stellar winds of the progenitor.

\section{Observations, reduction, and dataset}

\subsection{Observations}

\subsubsection{APEX}

A mosaic of the IC443G extended region was carried out with APEX $^{1}$. The APEX observations toward IC443G were conducted on September 11, 2018. The heterodyne receivers

\footnotetext{
1 This publication is partly based on data acquired under project M9508A_102 with the Atacama Pathfinder EXperiment (APEX). APEX is a collaboration between the Max-Planck-Institut für Radioastronomie (MPIfR), the European Southern Observatory, and the Onsala Space Observatory.
} 
P. Dell'Ova et al.: Interstellar anatomy of the TeV gamma-ray peak in the IC443 supernova remnant

Table 1. Spectroscopic parameters corresponding to the observed lines.

\begin{tabular}{lccccc}
\hline \hline Specie & $J_{\text {up }}$ & $v_{i j}(\mathrm{GHz})$ & $A_{i j}\left(\mathrm{~s}^{-1}\right)$ & $g_{\text {up }}$ & $E_{\text {up }}(\mathrm{K})$ \\
\hline${ }^{12} \mathrm{CO}$ & 1 & 115.2712018 & $7.203 \times 10^{-8}$ & 3 & 5.53 \\
${ }^{12} \mathrm{CO}$ & 2 & 230.5380000 & $6.910 \times 10^{-7}$ & 5 & 16.6 \\
${ }^{12} \mathrm{CO}$ & 3 & 345.7959899 & $2.497 \times 10^{-6}$ & 7 & 33.19 \\
\hline${ }^{13} \mathrm{CO}$ & 1 & 110.2013543 & $6.294 \times 10^{-8}$ & 3 & 5.29 \\
${ }^{13} \mathrm{CO}$ & 2 & 220.3986841 & $6.038 \times 10^{-7}$ & 5 & 15.87 \\
${ }^{13} \mathrm{CO}$ & 3 & 330.5879652 & $2.181 \times 10^{-6}$ & 7 & 31.73 \\
\hline $\mathrm{C}^{18} \mathrm{O}$ & 1 & 109.7821734 & $6.266 \times 10^{-8}$ & 3 & 5.27 \\
$\mathrm{C}^{18} \mathrm{O}$ & 2 & 219.5603541 & $6.011 \times 10^{-7}$ & 5 & 15.81 \\
\hline
\end{tabular}

Notes. The quantity $J_{\text {up }}$ is the rotational quantum number, $v_{i j}$ and $A_{i j}$ are the frequency and Einsten coefficient of the pure rotational transition, respectively $(v=0$, where $v$ is the vibrational quantum number) The quantities $E_{\text {up }}$ and $g_{\text {up }}$ are the energy and degeneracy corresponding to the upper level, respectively. The values given are taken from the Cologne Database for Molecular Spectroscopy (Müller et al. 2001, 2005; Endres et al. 2016) and Jet Propulsion Laboratory database (Pickett et al. 1998) and are the numeric values used for all our measures.

PI230 and FLASH345 (First Light APEX Submillimeter Heterodyne receiver; Heyminck et al. 2006), operating at 230 and $345 \mathrm{GHz}$, respectively, were used in combination with the FFTS4G and the Max-Planck-Institut für Radioastronomie (MPIfR) fast Fourier transform spectrometer backend (XFFTS; Klein et al. 2012). This setup allowed us to cover a total field of $10^{\prime} \times 10^{\prime}$ toward the center of the molecular region $\mathrm{G}$. The observations were performed in position-switching and on-thefly mode using APECS software (Muders et al. 2006). Table 1 contains the spectroscopic parameters of the observed lines and Table 2 contains the corresponding observing setups ${ }^{2}$.

The central position of all observations was $\alpha_{\text {[J2000] }}=6^{\mathrm{h}} 16^{\mathrm{m}} 37.5^{\mathrm{s}}, \quad \delta_{[\mathrm{J} 2000]}=+22^{\circ} 35^{\prime} 00^{\prime \prime}$. The off-position used was $\alpha_{[\mathrm{J} 2000]}=6^{\mathrm{h}} 17^{\mathrm{m}} 35.8^{\mathrm{s}}, \delta_{[\mathrm{J} 2000]}=+22^{\circ} 33^{\prime} 08^{\prime \prime}$, in the inner region of the SNR. We checked the focus during the observing session on the stars IK Tau and R Dor. We checked line and continuum pointing every hour locally on V370 Aur, $\mathrm{Y}$ Tau, IK Tau, and R Dor. The pointing accuracy was better than $\sim 3^{\prime \prime}$ rms, regardless of which receiver we used. The absolute flux density scale was also calibrated on these sources. The absolute flux calibration uncertainty was estimated to be $\approx 15 \%$ during our observations. We used the GILDAS ${ }^{3}$ package to calibrate and merge the data of all subfields to produce a mosaic, and extract the spectral bands containing the signal corresponding to the $(2-1)$ rotational transitions of ${ }^{12} \mathrm{CO},{ }^{13} \mathrm{CO}$, $\mathrm{C}^{18} \mathrm{O}$, and the $(3-2)$ rotational transitions of ${ }^{12} \mathrm{CO}$ and ${ }^{13} \mathrm{CO}$. The reduction included first order baseline subtraction, spatial, and spectral regridding. The final products of this reduction process are spectral cubes centered on the previously cited rotational lines with a resolution of $0.5 \mathrm{~km} \mathrm{~s}^{-1}$ to increase the signal-to-noise ratio $(\mathrm{S} / \mathrm{N})$, which is more than enough for the

\footnotetext{
2 APEX telescope efficiencies are taken from http://www. apex-telescope.org/telescope/efficiency/index.php, and IRAM telescope efficiencies are taken from https://www. iram-institute.org/medias/uploads/eb2013-v8.2.pdf

3 The Grenoble Image and Line Data Analysis Software is developed and maintained by IRAM to reduce and analyze data obtained with the $30 \mathrm{~m}$ telescope and Plateau de Bure interferometer. See www . iram. fr/ IRAMFR/GILDAS
}

expected line widths (nominal spectral resolutions are indicated in Table 2). The observed area is shown in the white box in Fig. 1.

\subsubsection{IRAM $30 \mathrm{~m}$}

The same mosaic of the IC443G extended region was carried out with the IRAM $30 \mathrm{~m}^{4}$. The IRAM observations toward IC443G were conducted during one week, from February 20, 2019 to February 24, 2019. The heterodyne receiver Eight MIxer Receiver (EMIR; Carter et al. 2012), operating at 115 and $230 \mathrm{GHz}$ simultaneously, was used in combination with the FTS200 and Versatile SPectrometer Array (VESPA) backends. This setup allowed us to cover the same total field of $10^{\prime} \times 10^{\prime}$ toward IC443. The observations were performed in position-switching and on-the-fly mode. Table 2 contains the corresponding observing setups.

The central position of all observations was the same as that used for APEX observations, $\alpha_{[\mathrm{J} 2000]}=6^{\mathrm{h}} 16^{\mathrm{m}} 37.5^{\mathrm{s}}$, $\delta_{[\mathrm{J} 2000]}=+22^{\circ} 35^{\prime} 00^{\prime \prime}$. The off-position used was $\alpha_{[\mathrm{J} 2000]}=$ $6^{\mathrm{h}} 17^{\mathrm{m}} 54^{\mathrm{s}}, \quad \delta_{[\mathrm{J} 2000]}=+22^{\circ} 47^{\prime} 40^{\prime \prime}$ in the northeastern ionized region of the SNR. We checked the continuum pointing and focus every hour during the observing sessions on several bright stars. The pointing accuracy was better than $\sim 3^{\prime \prime}$ rms. The absolute flux calibration uncertainty was estimated to be $\approx 15 \%$. We also used the GILDAS package to calibrate and merge the data of all subfields to produce a mosaic, and extract the spectral bands containing the signal corresponding to the $(1-0)$ rotational transitions of ${ }^{12} \mathrm{CO},{ }^{13} \mathrm{CO}, \mathrm{C}^{18} \mathrm{O}$, and $(2-1)$ rotational transition of ${ }^{12} \mathrm{CO}$. The reduction process and final products are identical to what was presented in the previous subsection. The observed area is shown in the white box in Fig. 1.

\subsubsection{Comparison between the IRAM $30 \mathrm{~m}$ and APEX telescope data cubes}

We estimated the systematic errors between the IRAM $30 \mathrm{~m}$ and APEX based on the data cubes corresponding to the observation of the rotational transition ${ }^{12} \mathrm{CO}(2-1)$ by both telescopes. For a visual comparison of the two data cubes, the channel maps are given in Figs. 2 (IRAM $30 \mathrm{~m}$ ) and D.3 (APEX). We found no evidence of systematic pointing error between the two data cubes. We also performed a quantitative comparison of the two spectral cubes. First, we resampled the data cubes to get the same spatial and spectral resolution. The spatial resolution was set to the nominal resolution of APEX $\theta=28.7^{\prime \prime}$, and the spectral resolution was set to the nominal resolution of the IRAM $30 \mathrm{M}$ $\Delta v=0.5 \mathrm{~km} \mathrm{~s}^{-1}$. Then, in each frequency channel and every single pixel of the mosaic we compared the signal detected by the two telescopes, for which the signal is greater than $3 \sigma$. The results of this complete investigation are represented on a $T_{\mathrm{mb}}^{\mathrm{APEX}}$ vs. $T_{\mathrm{mb}}^{30 \mathrm{~m}} 2 \mathrm{D}$ histogram shown in Fig. 3. We determined the best linear fit $x \mapsto a \cdot x+b$ corresponding to the data dispersion between the two telescopes. The parameters given by the $\chi^{2}$ minimization are $a=0.88 ; b=-0.35$, indicating a slight overestimate in the measurement of the flux by the IRAM $30 \mathrm{~m}$ with respect to APEX measurements, at least in the scope of our observations. Thus, our measurements are characterized by a systematic error of approximately $12 \%$. This could be due

4 This work is based on observations out under project number 169-18 with the IRAM $30 \mathrm{~m}$ telescope. IRAM is supported by INSU/CNRS (France), MPG (Germany) and IGN (Spain). 
Table 2. Observed lines and corresponding telescope parameters for the observations of the extended G region of IC443.

\begin{tabular}{lccccccccc}
\hline \hline Species & ${ }^{12} \mathrm{CO}$ & ${ }^{12} \mathrm{CO}$ & ${ }^{12} \mathrm{CO}$ & ${ }^{12} \mathrm{CO}$ & ${ }^{13} \mathrm{CO}$ & ${ }^{13} \mathrm{CO}$ & ${ }^{13} \mathrm{CO}$ & $\mathrm{C}^{18} \mathrm{O}$ & $\mathrm{C}^{18} \mathrm{O}$ \\
\hline Line & $(1-0)$ & $(2-1)$ & $(2-1)$ & $(3-2)$ & $(1-0)$ & $(2-1)$ & $(3-2)$ & $(1-0)$ & $(2-1)$ \\
Telescope & IRAM 30 m & IRAM 30 m & APEX & APEX & IRAM 30 m & APEX & APEX & IRAM 30 m & APEX \\
\hline$v(\mathrm{GHz})$ & 115.271 & 230.538 & 230.538 & 345.796 & 110.201 & 220.399 & 330.588 & 109.782 & 219.560 \\
$F W H M\left({ }^{(\prime)}\right)$ & 22.5 & 11.2 & 28.7 & 19.2 & 23.5 & 30.1 & 20.0 & 23.6 & 30.2 \\
Sampling $\left({ }^{\prime \prime}\right)$ & 3.5 & 3.5 & 6 & 6 & 3.5 & 6 & 6 & 3.5 & 6 \\
\hline Receiver & EMIR & EMIR & PI230 & FLASH345 & EMIR & PI230 & FLASH345 & EMIR & PI230 \\
Obs. dates & $19-02-20$ & $19-02-20$ & $18-09-11$ & $18-09-11$ & $19-02-20$ & $18-09-11$ & $18-09-11$ & $19-02-20$ & $18-09-11$ \\
& $19-02-24$ & $19-02-24$ & $18-09-11$ & $18-09-11$ & $19-02-24$ & $18-09-11$ & $18-09-11$ & $19-02-24$ & $18-09-11$ \\
\hline$F_{\text {eff }}$ & 0.94 & 0.92 & 0.95 & 0.95 & 0.91 & 0.95 & 0.95 & 0.94 & 0.95 \\
$B_{\text {eff }}$ & 0.78 & 0.59 & 0.73 & 0.63 & 0.51 & 0.73 & 0.63 & 0.78 & 0.73 \\
\hline \hline$T_{\text {sys }}(\mathrm{K})$ & 172 & 321 & 146 & 291 & 118 & 129 & 330 & 118 & 129 \\
$\left.\Delta v(\mathrm{~km} \mathrm{~s})^{-1}\right)$ & 0.5 & 0.5 & 0.1 & 0.1 & 0.5 & 0.1 & 0.1 & 0.5 & 0.1 \\
rms $(\mathrm{K})$ & 0.035 & 0.039 & 0.069 & 0.066 & 0.025 & 0.084 & 0.080 & 0.022 & 0.130 \\
\hline
\end{tabular}

Notes. $v$ is the frequency of the transition, FWHM corresponds to the full width at half maximum of the instrumental beam, $F_{\text {eff }}$ is the forward efficiency, and $B_{\text {eff }}$ the beam efficiency of the dish. The quantity $\overline{T_{\text {sys }}}$ is the average system noise temperature and $\Delta v$ the nominal spectral resolution. PWV is the precipitable water vapor, and rms is the standard deviation measured on the baseline resampled with a spectral resolution of $0.5 \mathrm{~km} \mathrm{~s}^{-1}$ to allow direct comparison between the data from the IRAM $30 \mathrm{~m}$ and APEX telescopes.

to inaccurate correction of telescope efficiencies and/or absolute flux calibration. Also, according to the dispersion around the instrumental linear model, our measurements are affected by a random error characterized by a standard deviation of $305 \mathrm{mK}$.

\subsection{Morphology of the region}

Our $10^{\prime} \times 10^{\prime}, \sim 10-30^{\prime \prime}$ resolution maps of ${ }^{12} \mathrm{CO}$ and ${ }^{13} \mathrm{CO}$ in the extended $\mathrm{G}$ region provide a detailed picture of the morphology of molecular clumps. Figure 4 (left panel) shows the emission in ${ }^{12} \mathrm{CO}(2-1)$ integrated between $v=-40.0 \mathrm{~km} \mathrm{~s}^{-1}$ and $v=+30 \mathrm{~km} \mathrm{~s}^{-1}$ mapped with the IRAM $30 \mathrm{~m}$. This wide interval of velocity includes all the components of the signal that are detected within the bandpass of our observations toward the extended $\mathrm{G}$ region. This map reveals a structured region with two main molecular structures that are spatially separated. The first structure (bottom center in Fig. 4) has a peak integrated intensity of $578 \mathrm{~K} \mathrm{~km} \mathrm{~s}^{-1}$, which is a magnitude higher than the peak integrated intensity of the second structure (top right in Fig. 4) that is around $63 \mathrm{~K} \mathrm{~km} \mathrm{~s}^{-1}$.

Figure 2 shows the channel maps corresponding to the IRAM $30 \mathrm{~m}$ observations of the ${ }^{12} \mathrm{CO} J=2-1$ transition, which gives the finest spatial resolution of all our observations: $\theta=11.2^{\prime \prime}$, or $\sim 0.1 \mathrm{pc}$ at the adopted distance of $1.9 \mathrm{kpc}$. The channel maps corresponding to other transitions of ${ }^{12} \mathrm{CO}$ and its isotopolog ${ }^{13} \mathrm{CO}$ are also available in the appendix:

$-{ }^{12} \mathrm{CO}(1-0)$ mapped with the IRAM $30 \mathrm{~m}$ (Fig. D.1);

- ${ }^{13} \mathrm{CO}(1-0)$ mapped with the IRAM $30 \mathrm{~m}$ (Fig. D.2);

$-{ }^{12} \mathrm{CO}(2-1)$ mapped with APEX (Fig. D.3);

- ${ }^{12} \mathrm{CO}(3-2)$ mapped with APEX (Fig. D.4).

Several faint and sparse knots are detected over all the field of observations, especially around the systemic velocity of IC443 $v_{\mathrm{LSR}}=-4.5 \mathrm{~km} \mathrm{~s}^{-1}$ (Hewitt et al. 2006). These structures, noticeable between $v=-7.5 \mathrm{~km} \mathrm{~s}^{-1}$ and $v=-1.5 \mathrm{~km} \mathrm{~s}^{-1}$, might either correspond to a slice of turbulent medium driven by the $\mathrm{SN}$ shockwave and/or belong to the ambient gas associated with the NW-SE molecular cloud in which IC443G is embedded
(Lee et al. 2012). Other than that, the description of the region probed by our observations can be divided into the following six distinct structures:

1. Cloudlet: in the upper part of the field we observe a large $\left(\sim 5^{\prime} \times 2^{\prime}\right.$, i.e., $\left.\sim 2.8 \times 1.1 \mathrm{pc}\right)$ elongated cloudlet detected between $v=-7.0 \mathrm{~km} \mathrm{~s}^{-1}$ and $v=-5.5 \mathrm{~km} \mathrm{~s}^{-1}$ (indicated by the letter "A" in Fig. 2), which is also detected in ${ }^{12} \mathrm{CO}(3-2)$. This structure was labeled G1 by Zhang et al. (2010), as part of the double-peaked morphology of the extended $\mathrm{G}$ region. The ${ }^{13} \mathrm{CO}$ $J=1-0$ counterpart of this structure is much brighter than the other main structures in the field, and it is also detected in the transitions $J=2-1$ and $J=3-2$, and in $\mathrm{C}^{18} \mathrm{O} J=1-0$ and $J=2-1$. This structure was also presented and characterized by Lee et al. (2012) who proposed the label SC 03, among a total of 12 SCs (of size $\sim 1^{\prime}$ ) found in IC443.

2. Ring-like structure: a ring-like structure seemingly lying in the center of the field (indicated by the letter "B" in Fig. 2), appearing between $v=-5.5 \mathrm{~km} \mathrm{~s}^{-1}$ and $v=-4.5 \mathrm{~km} \mathrm{~s}^{-1}$ and also detected in ${ }^{12} \mathrm{CO}(3-2)$. It has a semimajor axis of $1.5^{\prime}$, or $0.8 \mathrm{pc}$. This structure might be spurious and is likely to be physically connected to the elongated cloudlet as both are spatially contiguous and their emission lines are spectrally close. It is partially detected as well in our observations of ${ }^{13} \mathrm{CO} J=1-0, J=2-1$ and $J=3-2$, and also has a faint, partial counterpart in $\mathrm{C}^{18} \mathrm{O}$ $J=1-0$ and $J=2-1$. To understand the nature of this region we searched for counterparts in Spitzer Multiband Imaging Photometer (MIPS), WISE, DSS and XMM-Newton data; and in near-infrared and optical point source catalogs (Sect. 5), without success. Owing to projection effects, this apparent circular shape could also be explained by an unresolved and clumpy distribution of gas.

3. Shocked clump: in the lower part of the field we identify a very bright clump emitting between $v=-31.0 \mathrm{~km} \mathrm{~s}^{-1}$ and $v=16 \mathrm{~km} \mathrm{~s}^{-1}$. This structure of size $\sim 2^{\prime} \times 0.75^{\prime}(\sim 1.1 \times 0.4 \mathrm{pc})$, which is detected in the ${ }^{12} \mathrm{CO}(3-2)$ transition as well, belongs to the southwestern ridge of the molecular shell of the SNR and has been described as a shocked molecular structure by several studies (van Dishoeck et al. 1993; Cesarsky et al. 1999; 


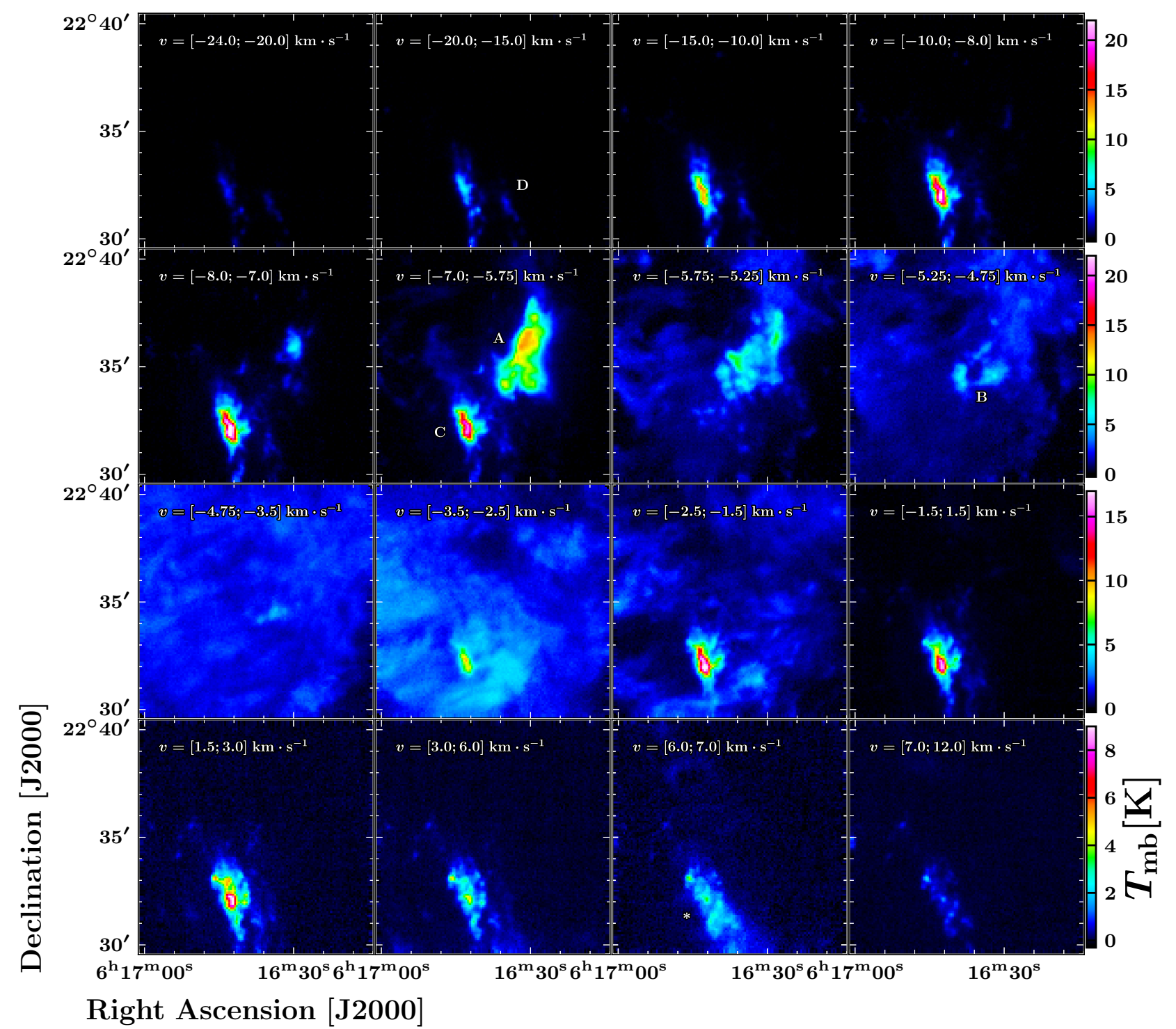

Fig. 2. Channel maps of the ${ }^{12} \mathrm{CO}(2-1)$ observations carried out with the IRAM $30 \mathrm{~m}$ telescope. Each panel represents the emission integrated over an interval of velocity along the line of sight. Velocity intervals are indicated on the top left corner of each panel. Velocity channels represented in this figure are between $v=-24 \mathrm{~km} \mathrm{~s}^{-1}$ and $v=+12 \mathrm{~km} \mathrm{~s}^{-1}$, covering all the spectral features detected toward the extended $\mathrm{G}$ region. Structures described in Sect. 3.2 are indicated with the corresponding letters.

Snell et al. 2005; Shinn et al. 2011; Zhang et al. 2010). The core of the shocked clump (indicated by the letter "C" in Fig. 2) is also detected in ${ }^{13} \mathrm{CO}$ in the transitions $J=1-0, J=2-1$, and $J=3-2$, and in $\mathrm{C}^{18} \mathrm{O} J=1-0$ and $J=2-1$.

4. Shocked knot: an additional shocked knot (indicated by the letter "D" in Fig. 2) is also detected to the west of the previously described structure. This fainter and smaller structure is spatially separated from the main shocked clump.

5. At the same position as the shocked clump and extending southward and westward, we find a faint, elongated clump emitting between $v=5.0 \mathrm{~km} \mathrm{~s}^{-1}$ and $v=7.5 \mathrm{~km} \mathrm{~s}^{-1}$. This structure (indicated by the symbol "*” in Fig. 2) spatially coincides with the shocked clump, yet the peak velocity is not exactly the same (see developments on kinematics of the region in Sect. 3.3). It has a faint counterpart in ${ }^{13} \mathrm{CO}(1-0)$. Observations of the ambient molecular cloud by Lee et al. (2012) indicate this structure as part of a faint NE-SW complex of molecular gas in the velocity range $+3 \mathrm{~km} \mathrm{~s}^{-1}<v_{\mathrm{LSR}}<+10 \mathrm{~km} \mathrm{~s}^{-1}$.
6. Finally, the ${ }^{13} \mathrm{CO}(1-0)$ map (Fig. 4, right panel and Fig. D.2) indicates a large clump of gas extending from the bottom center to the right end of the field, with a bright knot in the bottom right corner of the field. However, this structure has no bright, well-defined counterpart in any of the ${ }^{12} \mathrm{CO}$ transitions maps. It is spatially and kinematically correlated with the faint and diffuse ${ }^{12} \mathrm{CO} J=1-0$ and $J=2-1$ emission seen in the velocity range $-5.5 \mathrm{~km} \mathrm{~s}^{-1}<v_{\mathrm{LSR}}<-2 \mathrm{~km} \mathrm{~s}^{-1}$. From the comparison with the ${ }^{12} \mathrm{CO}$ observations of Lee et al. (2012) and ${ }^{13} \mathrm{CO}$ observations of Su et al. (2014) toward the SNR, we conclude that this structure is part of the western molecular complex observed in the velocity range $-10 \mathrm{~km} \mathrm{~s}^{-1}<v_{\mathrm{LSR}}<0 \mathrm{~km} \mathrm{~s}^{-1}$.

\subsection{Kinematics of the region}

Using the nominal spectral resolution of $0.5 \mathrm{~km} \mathrm{~s}^{-1}$ attained with our IRAM $30 \mathrm{~m}{ }^{12} \mathrm{CO} J=2-1$ observations, we identified several velocity components of the molecular gas in IC443G 


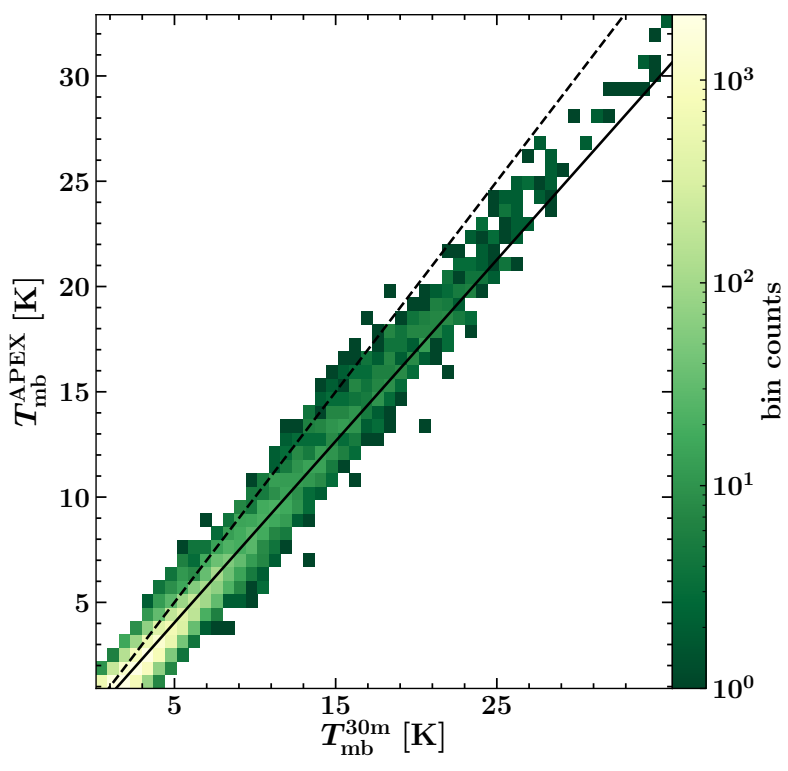

Fig. 3. Two-dimensional histogram of $T_{\mathrm{mb}}^{\mathrm{APEX}}$ vs. $T_{\mathrm{mb}}^{30 \mathrm{~m}} 2 \mathrm{D}$ representing the complete comparison between the data cubes obtained with the IRAM $30 \mathrm{~m}$ and APEX telescopes observations of the transition ${ }^{12} \mathrm{CO}(2-1)$ in the extended $\mathrm{G}$ region. The two cubes were resampled to the same spatial and spectral resolution. Every spectral channel in every single pixel is compared and shown in the histogram. The dashed black line represents the 1:1 relation expected between the two spectral cubes if the data was identical. The solid black line represents the empirical relation that is measured by determining the best linear fit corresponding to the data dispersion, using a threshold of $1.5 \mathrm{~K}$ to best describe the high signal-to-noise bins.

based on the determination of the first and second moment maps (Fig. 5, left and center) of the ${ }^{12} \mathrm{CO}(2-1)$ data cube. We also produced the moments maps of the ${ }^{12} \mathrm{CO}(3-2)$ data cube toward the ring-like structure (Fig. 5, right) to profit from the spectral resolution of $0.1 \mathrm{~km} \mathrm{~s}^{-1}$.

1. The cloudlet has a mean velocity of about $v_{\mathrm{LSR}}=$ $-5.5 \mathrm{~km} \mathrm{~s}^{-1}$ that is remarkably uniform throughout the structure. We measured $v_{\mathrm{LSR}}=-5.7 \pm 0.3 \mathrm{~km} \mathrm{~s}^{-1}$ from the centroid of the ${ }^{13} \mathrm{CO}$ lines, contrasting with the velocity of IC443 in the local standard of rest by more than $1 \mathrm{~km} \mathrm{~s}^{-1}$. It is likely that this discrepancy is due to a distinct velocity component with respect to the rest of the molecular gas in the extended $\mathrm{G}$ region. If this is not the case, this velocity shift could correspond to a maximum displacement of the kinematic distance $\Delta d \approx 300 \mathrm{pc}$ (following Wenger et al. 2018). Yet, the velocity wings of the ${ }^{12} \mathrm{CO}$ lines are still within the velocity range of the maser source in IC443G. The second moment map reveals a much lower velocity dispersion within the cloudet than for the shocked clump. It varies between $\sim 5$ and $\sim 7 \mathrm{~km} \mathrm{~s}^{-1}$, which is slightly higher than the velocity dispersion across the background field, around $\sim 4 \mathrm{~km} \mathrm{~s}^{-1}$

2. The apparent ring-like structure is further analyzed in the two right panels of Fig. 5, where the first and second moment maps are determined for the ${ }^{12} \mathrm{CO}(3-2)$ data cube obtained with APEX. The superior spectral resolution of the APEX data cube offers a better precision in the determination of the moment maps, at the cost of a lower spatial resolution. In the first moment map the mean velocity gradient within the ring suggests that the structure is rotating or expanding isotropically, as the mean velocity field varies between $v_{\mathrm{LSR}}=-4.7 \mathrm{~km} \mathrm{~s}^{-1}$ and $v_{\mathrm{LSR}}=-5.8 \mathrm{~km} \mathrm{~s}^{-1}$ from the western to the eastern arc of the ring. This apparent velocity gradient could be also due to systematic velocity variations between two or more distinct sub-clumps that are not well resolved by our $J=3-2$ observations $\left(\theta=19.2^{\prime \prime}\right.$, $\sim 0.2 \mathrm{pc})$. The velocity dispersion measured within the ring-like structure varies between 1 and $3 \mathrm{~km} \mathrm{~s}^{-1}$, with a positive gradient from the eastern part to the western part of the structure where it spatially connects to the cloudlet.

3 . The shocked clump has a mean velocity varying between $v_{\mathrm{LSR}}=-6 \mathrm{~km} \mathrm{~s}^{-1}$ and $v_{\mathrm{LSR}}=-8.5 \mathrm{~km} \mathrm{~s}^{-1}$ throughout its structure. We caution that these mean velocities are uncertain because the self-absorption and asymmetric wings characterizing the line emissions of ${ }^{12} \mathrm{CO}$ might bias the value of the centroid. In fact, careful measurement of the centroid of ${ }^{13} \mathrm{CO}$ lines using a single Gaussian function favors a velocity centroid of $-4.4 \pm 0.2 \mathrm{~km} \mathrm{~s}^{-1}$ for the shocked clump, which is consistent with the velocity $v_{\mathrm{LSR}}=-4.5 \mathrm{~km} \mathrm{~s}^{-1}$ of the maser in IC443G (Hewitt et al. 2006). The second moment map represents important velocity dispersions, spanning from $\sim 15$ and up to $\sim 36 \mathrm{~km} \mathrm{~s}^{-1}$ within the shocked gas, increasing toward the center of the clump.

4. The shocked knot has a mean velocity $v=-9 \mathrm{~km} \mathrm{~s}^{-1}$ that is slightly shifted with respect to the shocked clump. The second moment map shows a uniform velocity dispersion of $\sim 25 \mathrm{~km} \mathrm{~s}^{-1}$, similar to the dispersion measured within the main shocked molecular structure.

The rest of the field of observation has a quasi-uniform mean velocity of $\sim-4.1$ to $\sim-2.8 \mathrm{~km} \mathrm{~s}^{-1}$, which is slightly different than the mean velocity of IC443G in the local standard of rest but consistent with the ambient NW-SE molecular cloud in which IC443G is embedded (Lee et al. 2012). The velocity dispersion of this ambient gas spans from $<1$ to $\sim 10 \mathrm{~km} \mathrm{~s}^{-1}$ in a few areas where the velocity dispersion is locally enhanced, with an average of $\sim 4 \mathrm{~km} \mathrm{~s}^{-1}$. Excluding the contribution of the shocked structures and localized high-velocity dispersion knots, velocity dispersions measured from the ${ }^{12} \mathrm{CO} J=2-1$ line in the extended $\mathrm{G}$ field span a range of rms velocity $\sigma_{v}=0.4-1.3 \mathrm{~km} \mathrm{~s}^{-1}$ in the ambient gas, and $\sigma_{v}=1.2$ to $1.8 \mathrm{~km} \mathrm{~s}^{-1}$ toward the cloudlet. At a temperature of $10 \mathrm{~K}$, the thermal contribution is $\sigma_{v}=0.32 \mathrm{~km} \mathrm{~s}^{-1}$ and it is likely that small-scale motions within the complex of molecular gas contribute to the measured dispersion, hence the ambient cloud is mostly quiescent, with turbulent motions smaller than $1 \mathrm{~km} \mathrm{~s}^{-1}$. The velocity dispersion measured toward the cloudlet with the ${ }^{13} \mathrm{CO}$ lines is $\sigma_{v}=0.8 \pm 0.1 \mathrm{~km} \mathrm{~s}^{-1}$, which is consistent with typical molecular condensations (Larson 1981). Thus, we do not find any kinematic signature of interaction of the cloudlet nor the ambient cloud with the SNR shocks in the extended $\mathrm{G}$ region, except for the few localized high-velocity dispersion knots.

\section{Column density and mass measurements}

\subsection{Spatial separation of spectrally uniform structures}

We aim to measure the mass associated with each molecular structures described in the previous section. We defined spatial boundaries enclosing these structures and independently studied the spectral data corresponding to each subregion of the field. The spatial boxes defined for the cloudlet (A), ring-like structure (B), shocked clump (C), and shocked knot (D) are shown in Fig. 6, and the average spectra obtained in these boxes are presented in Figs. 8 and 7 for every line from $\mathrm{CO}$ and its isotopologs, which are available in our IRAM $30 \mathrm{~m}$ and APEX data cubes. The choice of the boundaries is based on our morphological classification, but we carefully checked that the brightest 

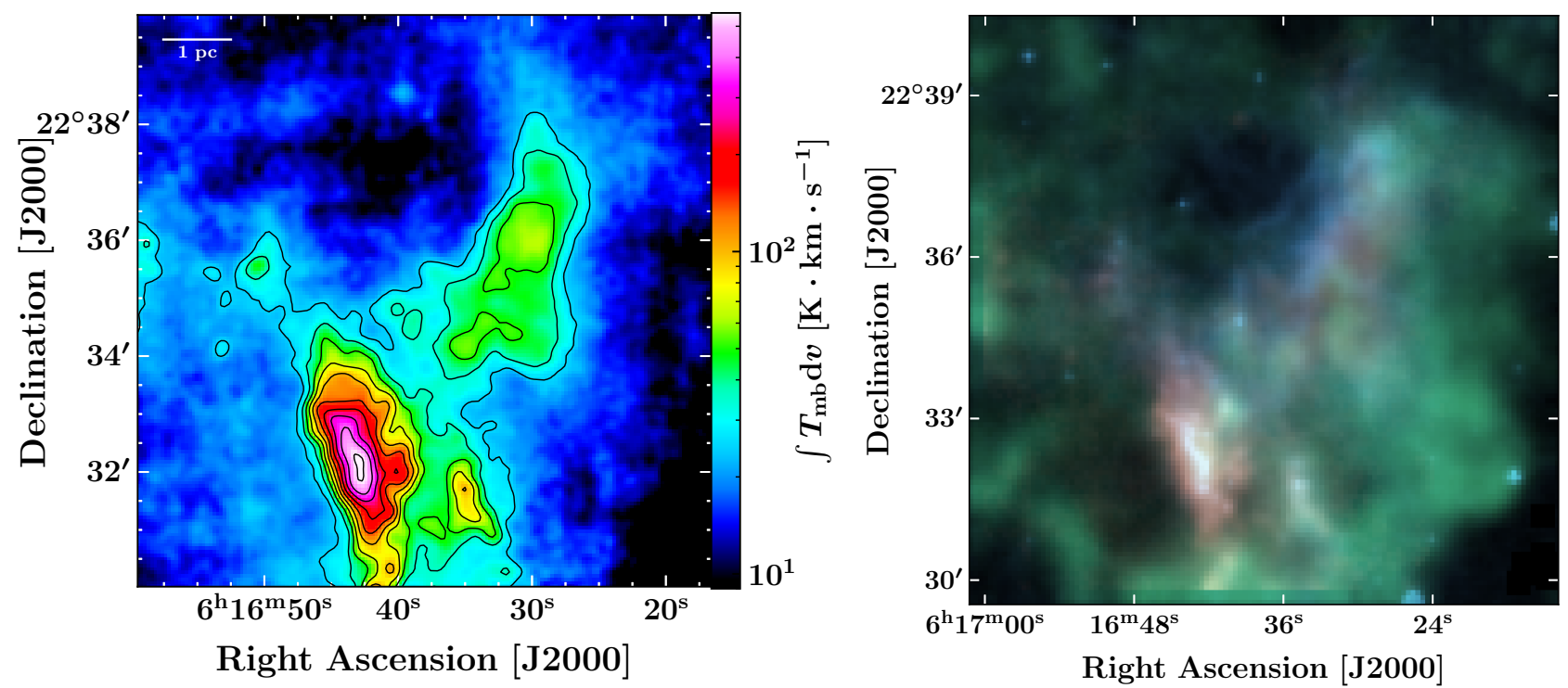

Fig. 4. Left: 0th moment of the ${ }^{12} \mathrm{CO}(2-1)$ observations carried out with the IRAM $30 \mathrm{~m}$ over the extended G region. This map corresponds to the signal integrated in the velocity interval $[-40 ;+30] \mathrm{km} \mathrm{s}^{-1}$. The color scale used to represent data is logarithmic to enhance the dynamic range and emphasize the fainter molecular cloudlet. Right: composite image of our field of observations in the extended G region, using an IRAM $30 \mathrm{~m}$ data cube as well as Spitzer-MIPS data; ${ }^{12} \mathrm{CO}(2-1)\left([-40,+30] \mathrm{km} \mathrm{s}^{-1}\right.$ as in the left panel) is coded in red, MIPS-24 $\mu \mathrm{m}$ in blue, and ${ }^{13} \mathrm{CO}(1-0)[-4.0$, $-2.5] \mathrm{km} \mathrm{s}^{-1}$, corresponding to the emission spatially and kinematically associated with the ambient cloud described by Lee et al. (2012) in green. Color scale levels are based on the minimum and maximum value of each map.
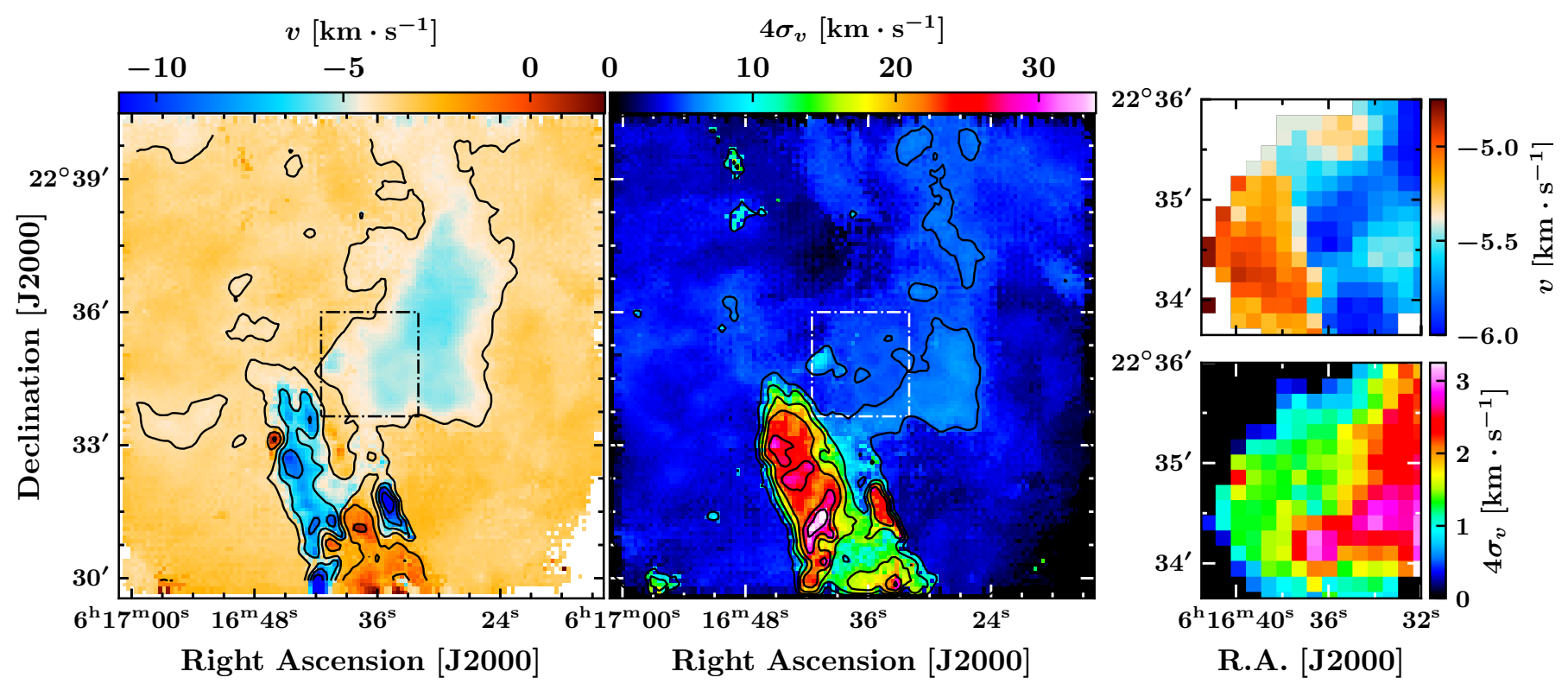

Fig. 5. Left: first moment map of the IRAM $30 \mathrm{~m}^{12} \mathrm{CO}(2-1)$ data cube. Center: second moment map of the same data cube. Right, top panel: zoom into the dashed box on the first moment map of the APEX ${ }^{12} \mathrm{CO}(3-2)$ data cube to enhance the spectral resolution. Right top and bottom panels: first and second moment maps of the APEX ${ }^{12} \mathrm{CO}(3-2)$ data cube into the dashed box shown in the right and middle panels, respectively. The color bar of the left figure is centered on the velocity of IC443 in the local standard of rest, $v_{\mathrm{LSR}}=-4.5 \mathrm{~km} \mathrm{~s}^{-1}$.

spectral features are coherent across the different boxes that we defined (coordinates of these boxes are given in Table A.1). We performed that selection manually, as the size of our sample is not large enough to apply statistical methods (e.g., clustering, see Bron et al. 2018). Based on the analysis of the emission of ${ }^{12} \mathrm{CO}$, ${ }^{13} \mathrm{CO}$ and $\mathrm{C}^{18} \mathrm{O}$ lines, our description of these spectral features is the following:

1. Cloudlet: toward box A (Fig. 7, left-panel), the line profile of ${ }^{12} \mathrm{CO}$ and ${ }^{13} \mathrm{CO}$ lines are similarly double peaked and best modeled by the sum of two Gaussian functions centered on the systemic velocities $v_{\mathrm{LSR}}=-5.7 \pm 0.3 \mathrm{~km} \mathrm{~s}^{-1}$ (associated with the cloudlet) and $v_{\mathrm{LSR}}=-3.3 \pm 0.1 \mathrm{~km} \mathrm{~s}^{-1}$ (associated with the ambient cloud).

2. Ring-like structure: toward box B (Fig. 7, right-panel), the ${ }^{12} \mathrm{CO}$ and ${ }^{13} \mathrm{CO}$ lines are double peaked as well. The use of two Gaussian functions to model the line profile yields the systemic velocities $v_{\mathrm{LSR}}=-5.6 \pm 0.2 \mathrm{~km} \mathrm{~s}^{-1}$ (associated with the ring-like structure) and $v_{\mathrm{LSR}}=-3.3 \pm 0.1 \mathrm{~km} \mathrm{~s}^{-1}$ (associated with the ambient cloud). The Gaussian decomposition is very similar to that of the cloudlet, suggesting that the apparent ring-like 


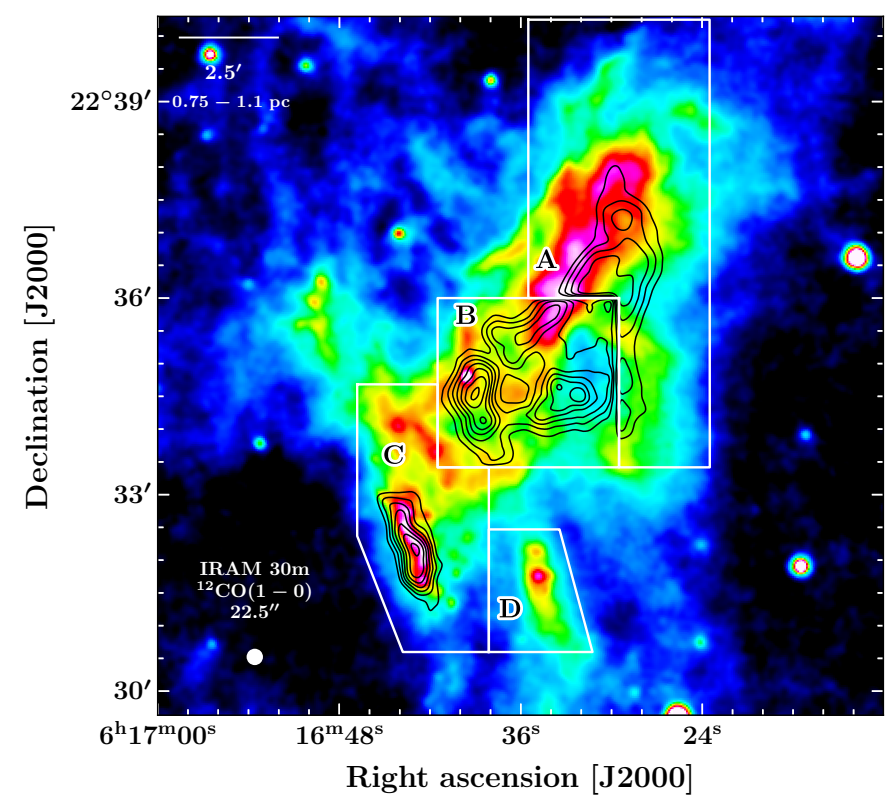

Fig. 6. Spitzer/MIPS map at $24.0 \mu \mathrm{m}$. In black contours, the emission of ${ }^{12} \mathrm{CO}(2-1)$ observed with the IRAM $30 \mathrm{~m}$ is shown over different intervals of velocities: A $[-7 ;-5] \mathrm{km} \mathrm{s}^{-1}$ (cloudlet); B $[-5.5 ;-4.5] \mathrm{km} \mathrm{s}^{-1}$ (ring-like structure); and C and D $[-40 ;+30] \mathrm{km} \mathrm{s}^{-1}$ (shocked clump and shocked knot). The white boxes represent the area where the signal corresponding to each structure is integrated. The beam diameter of the IRAM $30 \mathrm{~m}$ observations of ${ }^{12} \mathrm{CO}(2-1)$ is shown in the bottom left corner.

structure might be incidental despite its remarkable features in the first moment map (Fig. 5).

3. Shocked clump: considering the geometry of the SNR and the locally perpendicular direction of propagation of the SNR shockwave (van Dishoeck et al. 1993), the high-velocity emission arises from at least two shock waves, if not a collection of transverse shocks propagating along the molecular shell. In other words, the projection along the line of sight of several distinct shocked knots with distinct systematic velocities could contribute to the broadening of the ${ }^{12} \mathrm{CO}$ lines. We measure $v_{\mathrm{s}} \simeq 27 \mathrm{~km} \mathrm{~s}^{-1}$ and $v_{\mathrm{s}} \simeq 21 \mathrm{~km} \mathrm{~s}^{-1}$ for the blueshifted and redshifted transverse shocks, respectively. Except for the $J=1-0$ spectrum for which the emission of the ambient gas contributes to the average spectra, all spectra of ${ }^{12} \mathrm{CO}$ lines exhibit a significant absorption feature around the $v_{\mathrm{LSR}}$ of IC443G, suggesting that there is strong self-absorption of the emission lines. Evidence of line absorption is found in the velocity range $-6 \mathrm{~km} \mathrm{~s}^{-1}<v_{\mathrm{LSR}}<-2 \mathrm{~km} \mathrm{~s}^{-1}$, which is where we detect the spatially extended features associated with the NW-SE complex of molecular gas described by Lee et al. (2012). Hence, it is possible that the foreground cold molecular cloud is at the origin of the absorption of the ${ }^{12} \mathrm{CO} J=1-0$ and $J=2-1$ lines. A faint and thin emission line is detected around $v=6.5 \mathrm{~km} \mathrm{~s}^{-1}$ both in the ${ }^{12} \mathrm{CO}$ and ${ }^{13} \mathrm{CO}$ spectra. This signal is associated with the NE-SW complex of molecular gas described in Sect. 3.2.

4. Shocked knot: the shock signature of this line is distinct from the shocked clump. As hinted by the moments map (Fig. 5), its fainter high-velocity wings are displaced toward negative velocities. A self-absorption feature is also observed in this structure. Between $v=-5.5 \mathrm{~km} \mathrm{~s}^{-1}$ and $v=-2 \mathrm{~km} \mathrm{~s}^{-1}$ a bright and thin feature traces the ambient gas shown on the channel maps in Fig. 2 (second row from bottom; first, second and third panels from left).
The line width measured from the ${ }^{13} \mathrm{CO}$ line profiles when we consider only the spectral component that are physically associated with the cloudlet and ring-like structure (discarding the contribution of the ambient cloud) are $2.0 \pm 0.3 \mathrm{~km} \mathrm{~s}^{-1}$ and $1.6 \pm 0.3 \mathrm{~km} \mathrm{~s}^{-1}$, respectively, measured by carefully defining much more constrained spatial boundaries around the structures. From the average spectra presented in this section, there is no spectral evidence for the propagation of molecular shocks and/or outflows within these two structures, except for the faint wings displayed by the ${ }^{12} \mathrm{CO}$ lines in the box associated with the ring-like structure. These extended wings arise from the contamination by the high-velocity emission of the shocked substructures that are contained in box B (Fig. 5). We measured the peak temperature, velocity centroid, FWHM, and area of the ${ }^{12} \mathrm{CO}$ and ${ }^{13} \mathrm{CO}$ lines $J=1-0, J=2-1$ and $J=3-2$ in each average spectrum and report our results in Table 3 .

\subsection{LTE method}

\subsubsection{Two-dimensional histograms of $\mathrm{CO}$ data}

In the next section, we aim to build population diagrams in which we correct the effect of optical depth on the column density of upper levels. To measure the optical depth of CO lines, we relied on several strong assumptions, in particular the adopted isotopic ratios and the identity of excitation temperature for ${ }^{12} \mathrm{CO}$ and ${ }^{13} \mathrm{CO}$ (see Sect. 4.2.2 for a description of our method, and Roueff et al. 2020 for a complete discussion of the corresponding assumptions). To assess the validity of this approach and estimate the key parameters (isotopic ratios and excitation temperature ratios), we built $2 \mathrm{D}$ histograms from the ${ }^{12} \mathrm{CO} J=1-0$, $J=2-1, J=3-2$ data cubes, as well as ${ }^{13} \mathrm{CO}$ and $\mathrm{C}^{18} \mathrm{O} J=1-0$ data cubes to compare the line intensity from different isotopologs and rotational transitions of $\mathrm{CO}$ with modified LTE models (Bron et al. 2018). These assumptions are also discussed in Sect. 4.2.3. We examined four different 2D data histograms:

1. $J=1-0$ line intensity, ${ }^{13} \mathrm{CO}$ vs. ${ }^{12} \mathrm{CO}$.

2. $J=1-0$ line intensity, ${ }^{13} \mathrm{CO}$ vs. $\mathrm{C}^{18} \mathrm{O}$.

3. $J=1-0$ line intensity ratio, $\left[{ }^{13} \mathrm{CO} / \mathrm{C}^{18} \mathrm{O}\right]$ vs. $\left[{ }^{12} \mathrm{CO} /{ }^{13} \mathrm{CO}\right]$.

4. ${ }^{12} \mathrm{CO}$ line intensity ratio, $[3-2] /[2-1]$ vs. $[2-1] /[1-0]$.

In order to build the first three data histograms, we convolved all IRAM $30 \mathrm{~m}$ data cubes to the nominal spatial resolution of $\mathrm{C}^{18} \mathrm{O}(1-0)$ (i.e., 23.6") and to the nominal spectral resolution of the FTS backend (i.e., $0.5 \mathrm{~km} \mathrm{~s}^{-1}$ ). To build the fourth histogram, we resampled IRAM $30 \mathrm{~m}{ }^{12} \mathrm{CO}(1-0)$, APEX ${ }^{12} \mathrm{CO}(2-1)$, and APEX ${ }^{12} \mathrm{CO}(3-2)$ data cubes to the nominal spatial resolution of ${ }^{12} \mathrm{CO}(1-0)$ (i.e., 22.5") and to the nominal spectral resolution given by the FTS backend (i.e., $0.5 \mathrm{~km} \mathrm{~s}^{-1}$ ). We used a threshold of $3 \sigma$ to select data points for which the signal is significantly above the noise level. The resulting 2D data histograms are shown in Fig. 9.

Results. The first histogram is characterized by a high $(\mathrm{S} / \mathrm{N})$ ratio and represents a large statistical sample $(n=20531)$. The ${ }^{13} \mathrm{CO}(1-0)$ vs. ${ }^{12} \mathrm{CO}(1-0)$ relation presents at least two distinct branches. The lower quasi-horizontal branch, tracing bright ${ }^{12} \mathrm{CO}(1-0)$ emission associated with faint ${ }^{13} \mathrm{CO}(1-0)$ line emission $\left(T_{13}<1 \mathrm{~K} \mathrm{~km} \mathrm{~s}^{-1}\right)$. This branch is spatially correlated with the shock structure and spectrally correlated with the highvelocity wings of the ${ }^{12} \mathrm{CO}$ line, which have no bright ${ }^{13} \mathrm{CO}$ counterpart because of insufficient integration time. It is spatially correlated mainly with the quiescent molecular gas that is found within the cloudlet and ring-like structure and the ambient cloud. 

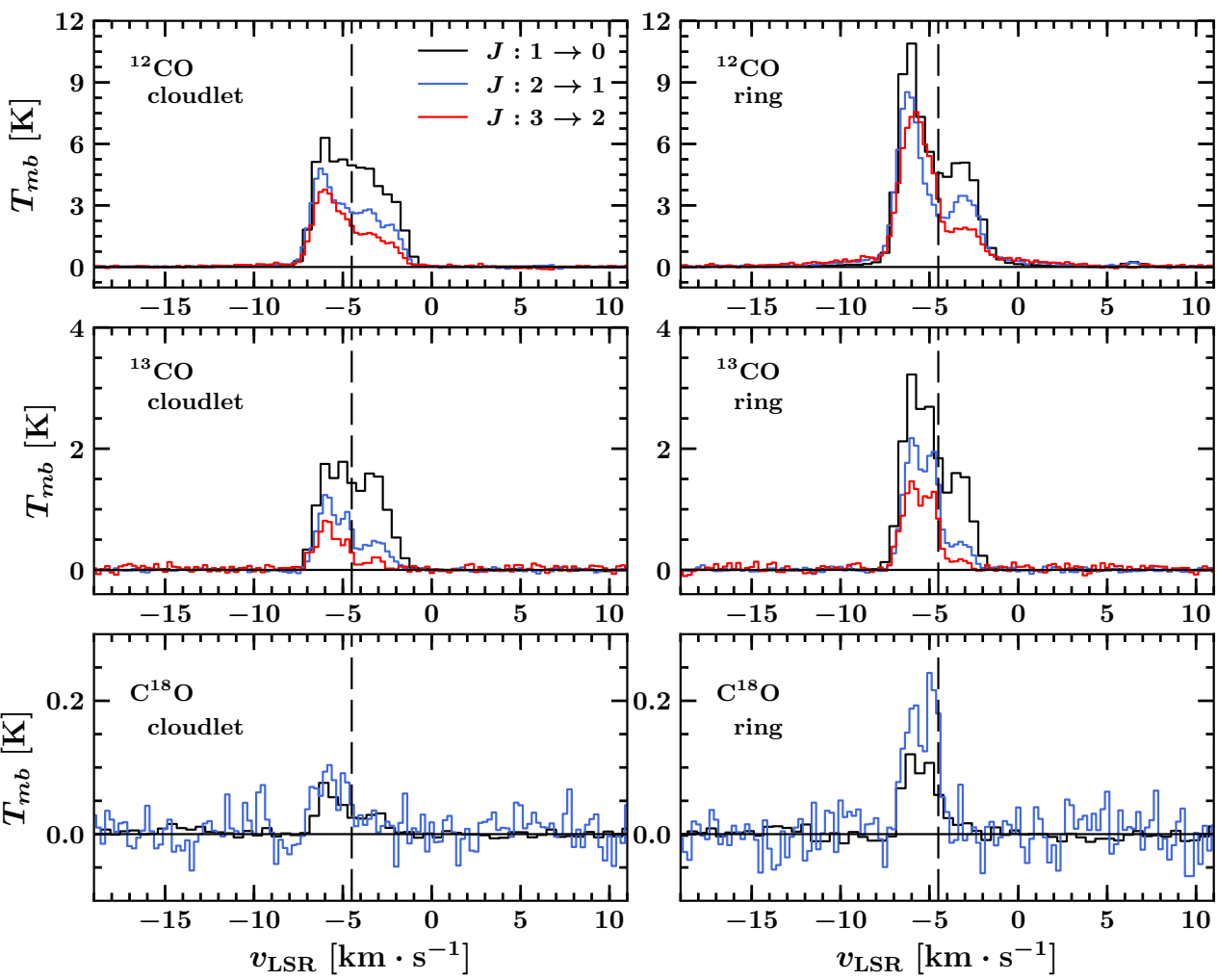

Fig. 7. Spectra averaged over the regions of the cloudlet (left panels) and the ring (right panels) defined in Fig. 6 for the following lines: ${ }^{12} \mathrm{CO}(1-0),{ }^{13} \mathrm{CO}(1-0)$ and $\mathrm{C}^{18} \mathrm{O}(1-0)$ (in black, IRAM-30m); ${ }^{12} \mathrm{CO}(2-1),{ }^{13} \mathrm{CO}(2-1)$ and $\mathrm{C}^{18} \mathrm{O}(2-1)$ (in blue, APEX); ${ }^{12} \mathrm{CO}(3-2)$ and ${ }^{13} \mathrm{CO}(3-2)$ (in grey, APEX). Spectral cubes were resampled to allow a direct comparison between the different spectra. Spatial resolutions of all transitions were modified to the nominal resolution of $\mathrm{C}^{18} \mathrm{O}(2-1), \quad \theta=30.2^{\prime \prime}$. Spectral resolutions were set to $0.5 \mathrm{~km} \mathrm{~s}^{-1}$ for IRAM $30 \mathrm{~m}$ data and $0.25 \mathrm{~km} \mathrm{~s}^{-1}$ for APEX data. On both panels, the $v_{\text {LSR }}$ of IC443 is indicated with a vertical dashed line $\left(\right.$ at $\left.-4.5 \mathrm{~km} \mathrm{~s}^{-1}\right)$.

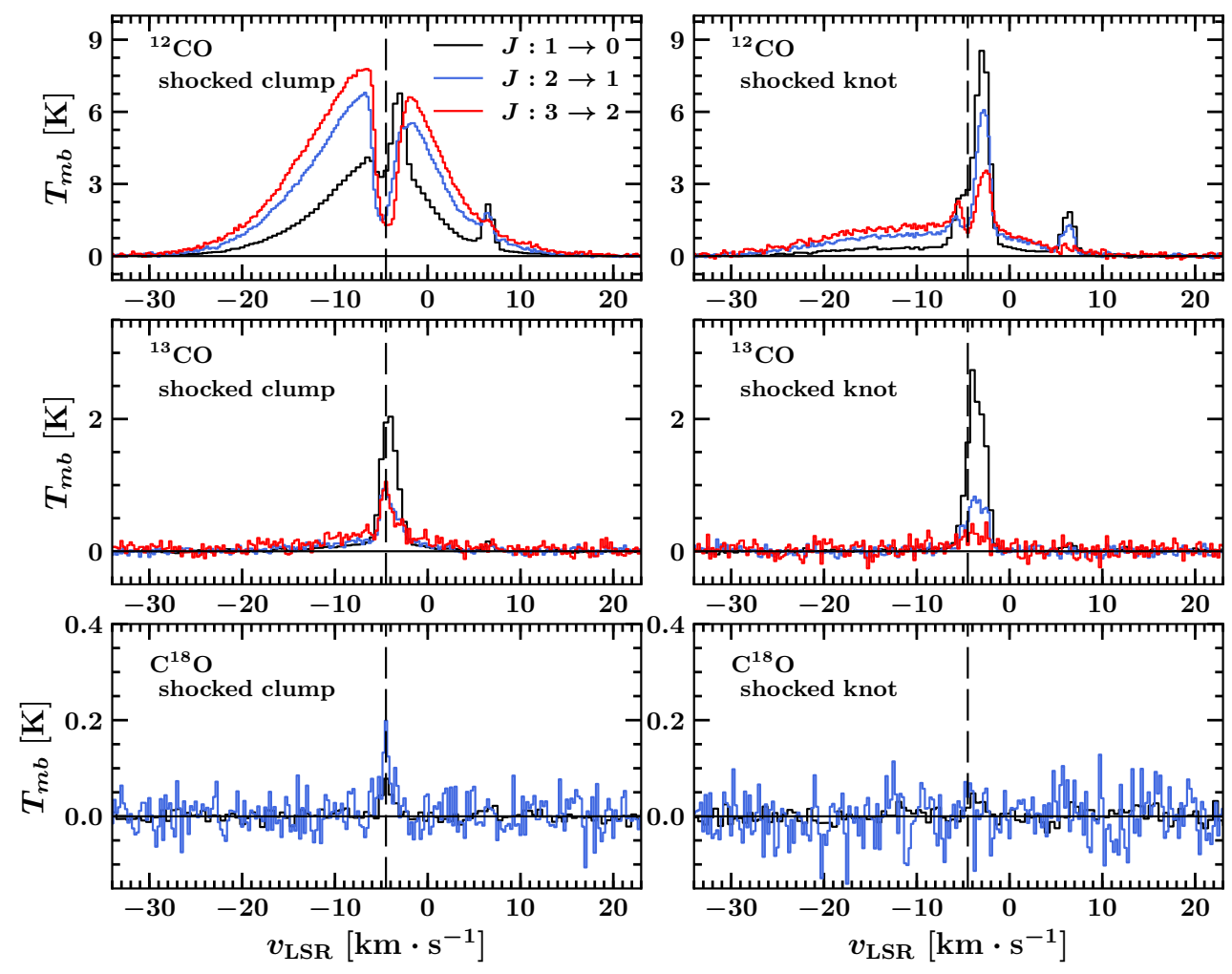

Fig. 8. Spectra averaged over the region of the shocked clump (left) and shocked knot (right) defined in Fig. 6 for the following lines: ${ }^{12} \mathrm{CO}(1-0),{ }^{13} \mathrm{CO}(1-0)$ and $\mathrm{C}^{18} \mathrm{O}(1-0)$ (in black, IRAM $\left.30 \mathrm{~m}\right) ;{ }^{12} \mathrm{CO}(2-1),{ }^{13} \mathrm{CO}(2-1)$ and $\mathrm{C}^{18} \mathrm{O}(2-1)$ (in blue, $\left.\mathrm{APEX}\right) ;{ }^{12} \mathrm{CO}(3-2)$ and ${ }^{13} \mathrm{CO}(3-2)$ (in grey, APEX). Spectral cubes were resampled to allow a direct comparison between the different spectra. Spatial resolutions of all transitions were modified to the nominal resolution of $\mathrm{C}^{18} \mathrm{O}(2-1), \theta=30.2^{\prime \prime}$. Spectral resolutions were set to $0.5 \mathrm{~km} \mathrm{~s}^{-1}$ for IRAM $30 \mathrm{~m}$ data and $0.25 \mathrm{~km} \mathrm{~s}^{-1}$ for APEX data. On both panels, the $v_{\text {LSR }}$ of IC443 is indicated with a vertical dashed line (at $-4.5 \mathrm{~km} \mathrm{~s}^{-1}$ ). 
Table 3. Summary of the spectral characteristics of the lines of ${ }^{12} \mathrm{CO}$ and ${ }^{13} \mathrm{CO}$ within the boxes corresponding to each structures, defined in Fig. 6 .

\begin{tabular}{|c|c|c|c|c|c|c|c|c|c|}
\hline \multirow[b]{2}{*}{ Region } & \multirow[b]{2}{*}{$J_{\mathrm{u}}-J_{1}$} & \multicolumn{4}{|c|}{${ }^{12} \mathrm{CO}$} & \multicolumn{4}{|c|}{${ }^{13} \mathrm{CO}$} \\
\hline & & $\begin{array}{l}T_{\mathrm{mb}}^{*} \\
(\mathrm{~K})\end{array}$ & $\begin{array}{c}v_{0} \\
\left(\mathrm{~km} \mathrm{~s}^{-1}\right)\end{array}$ & $\begin{array}{c}v_{\mathrm{FWHM}} \\
\left(\mathrm{km} \mathrm{s}^{-1}\right)\end{array}$ & $\begin{array}{c}\text { Area } \\
\left(\mathrm{K} \mathrm{km} \mathrm{s}^{-1}\right)\end{array}$ & $\begin{array}{l}T_{\mathrm{mb}}^{*} \\
(\mathrm{~K})\end{array}$ & $\begin{array}{c}v_{0} \\
\left(\mathrm{~km} \mathrm{~s}^{-1}\right)\end{array}$ & $\begin{array}{c}v_{\mathrm{FWHM}} \\
\left(\mathrm{km} \mathrm{s}^{-1}\right)\end{array}$ & $\begin{array}{c}\text { Area } \\
\left(\mathrm{K} \mathrm{km} \mathrm{s}^{-1}\right)\end{array}$ \\
\hline $\begin{array}{l}\text { Cloudlet } \\
\alpha=6^{\mathrm{h}} 16^{\mathrm{m}} 30^{\mathrm{s}} \\
\delta=22^{\circ} 36^{\prime} 00^{\prime \prime}\end{array}$ & $\begin{array}{l}1-0 \\
2-1 \\
3-2\end{array}$ & $\begin{array}{l}6.1 \pm 0.1 \\
4.7 \pm 0.1 \\
3.5 \pm 0.1\end{array}$ & $\begin{array}{l}-5.8 \pm 0.2 \\
-5.9 \pm 0.3 \\
-6.1 \pm 0.2\end{array}$ & $\begin{array}{l}2.1 \pm 0.3 \\
1.9 \pm 0.3 \\
1.6 \pm 0.2\end{array}$ & $\begin{array}{c}15.5 \pm 0.3 \\
9.7 \pm 0.2 \\
6.9 \pm 0.2\end{array}$ & $\begin{array}{l}1.7 \pm 0.1 \\
1.1 \pm 0.1 \\
0.8 \pm 0.1\end{array}$ & $\begin{array}{l}-5.6 \pm 0.2 \\
-5.7 \pm 0.3 \\
-5.8 \pm 0.3\end{array}$ & $\begin{array}{l}2.0 \pm 0.2 \\
1.8 \pm 0.2 \\
2.2 \pm 0.3\end{array}$ & $\begin{array}{l}4.1 \pm 0.1 \\
1.7 \pm 0.3 \\
1.2 \pm 0.1\end{array}$ \\
\hline $\begin{array}{l}\text { Ring } \\
\alpha=6^{\mathrm{h}} 16^{\mathrm{m}} 36^{\mathrm{s}} \\
\delta=22^{\circ} 34^{\prime} 40^{\prime \prime}\end{array}$ & $\begin{array}{l}1-0 \\
2-1 \\
3-2\end{array}$ & $\begin{array}{c}11.7 \pm 0.2 \\
9.5 \pm 0.1 \\
9.5 \pm 0.1\end{array}$ & $\begin{array}{l}-6.0 \pm 0.2 \\
-6.1 \pm 0.1 \\
-5.7 \pm 0.3\end{array}$ & $\begin{array}{l}1.6 \pm 0.2 \\
1.4 \pm 0.2 \\
1.9 \pm 0.2\end{array}$ & $\begin{array}{l}20.1 \pm 0.3 \\
14.3 \pm 0.2 \\
15.0 \pm 0.3\end{array}$ & $\begin{array}{l}3.5 \pm 0.1 \\
2.8 \pm 0.1 \\
1.8 \pm 0.1\end{array}$ & $\begin{array}{l}-5.7 \pm 0.2 \\
-5.5 \pm 0.2 \\
-5.5 \pm 0.3\end{array}$ & $\begin{array}{l}1.7 \pm 0.2 \\
1.6 \pm 0.2 \\
1.7 \pm 0.3\end{array}$ & $\begin{array}{l}5.8 \pm 0.2 \\
3.6 \pm 0.1 \\
2.5 \pm 0.1\end{array}$ \\
\hline $\begin{array}{l}\text { Shocked clump } \\
\alpha=6^{\mathrm{h}} 16^{\mathrm{m}} 42^{\mathrm{s}} \\
\delta=22^{\circ} 32^{\prime} 00^{\prime \prime}\end{array}$ & $\begin{array}{l}1-0 \\
2-1 \\
3-2\end{array}$ & $\begin{array}{l}7.1 \pm 0.1 \\
5.2 \pm 0.1 \\
4.9 \pm 0.1\end{array}$ & $\begin{array}{l}-6 \pm 1 \\
-7 \pm 1 \\
-7 \pm 1\end{array}$ & $\begin{array}{l}19 \pm 1 \\
23 \pm 1 \\
24 \pm 1\end{array}$ & $\begin{array}{c}49.7 \pm 0.4 \\
71 \pm 1 \\
80 \pm 1\end{array}$ & $\begin{array}{l}2.5 \pm 0.1 \\
0.8 \pm 0.1 \\
0.7 \pm 0.1\end{array}$ & $\begin{array}{l}-3.8 \pm 0.1 \\
-3.8 \pm 0.2 \\
-4.4 \pm 0.2\end{array}$ & $\begin{array}{l}2.1 \pm 0.1 \\
2.4 \pm 0.2 \\
1.4 \pm 0.2\end{array}$ & $\begin{array}{l}6.5 \pm 0.1 \\
3.5 \pm 0.1 \\
4.5 \pm 0.2\end{array}$ \\
\hline $\begin{array}{l}\text { Shocked knot } \\
\alpha=6^{\mathrm{h}} 16^{\mathrm{m}} 42^{\mathrm{s}} \\
\delta=22^{\circ} 32^{\prime} 00^{\prime \prime}\end{array}$ & $\begin{array}{l}1-0 \\
2-1 \\
3-2\end{array}$ & $\begin{array}{l}8.8 \pm 0.1 \\
6.0 \pm 0.1 \\
3.4 \pm 0.1\end{array}$ & $\begin{array}{l}-8 \pm 2 \\
-9 \pm 2 \\
-9 \pm 2\end{array}$ & $\begin{array}{l}17 \pm 1 \\
21 \pm 1 \\
22 \pm 1\end{array}$ & $\begin{array}{l}18.8 \pm 0.2 \\
21.1 \pm 0.3 \\
24.4 \pm 0.4\end{array}$ & $\begin{array}{l}2.4 \pm 0.1 \\
0.7 \pm 0.1 \\
0.3 \pm 0.1\end{array}$ & $\begin{array}{l}-3.7 \pm 0.1 \\
-3.5 \pm 0.2 \\
-3.9 \pm 0.3\end{array}$ & $\begin{array}{l}2.4 \pm 0.1 \\
2.6 \pm 0.3 \\
1.9 \pm 0.3\end{array}$ & $\begin{array}{l}7.5 \pm 0.1 \\
3.2 \pm 0.1 \\
1.3 \pm 0.5\end{array}$ \\
\hline
\end{tabular}

Notes. $T_{\mathrm{mb}}^{*}$ is the peak temperature of the line, $v_{0}$ is the centroid, and $v_{\mathrm{FWHM}}$ the line width. The quantity $T_{\mathrm{mb}}^{*}$ and the area are directly measured from reading the spectral data. The quantities $v_{\mathrm{FWHM}}$ and $v_{0}$ are measured by modeling the line either by a single or double Gaussian function that best fits the data. When two Gaussian functions are needed to model the sum of the signal emitted by the ambient cloud and structure of interest, we discard the contribution of the ambient cloud and only take into account the parameters $v_{0}$ and $v_{\mathrm{FWHM}}$ associated with the broad component. The asymmetric high-velocity wings of ${ }^{12} \mathrm{CO}$ lines corresponding to the shocked clump require two Gaussians, thus we measure $v_{0}=\left(c_{1}+c_{2}\right) / 2$ and $v_{\mathrm{FWHM}}=\sqrt{\left(\left(2.355 \sigma_{1}\right)^{2}+\left(2.355 \sigma_{2}\right)^{2}\right)}$, where $c_{1}, c_{2}, \sigma_{1}$, and $\sigma_{2}$ are the centroids and standard deviations corresponding to each Gaussian function, respectively.
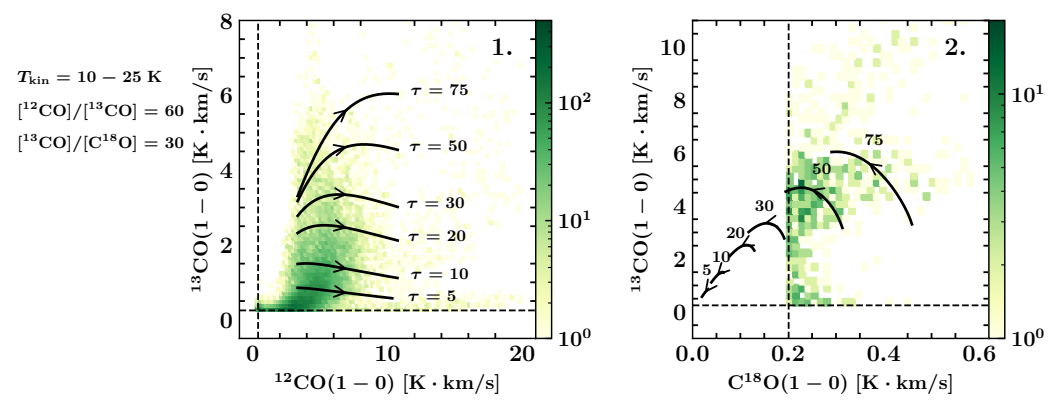

$$
\begin{aligned}
& T_{\text {kin }}=10-25 \mathrm{~K} \\
& {\left[{ }^{12} \mathrm{CO}\right] /\left[{ }^{13} \mathrm{CO}\right]=60} \\
& {\left[{ }^{13} \mathrm{CO}\right] /\left[\mathrm{C}^{18} \mathrm{O}\right]=30}
\end{aligned}
$$
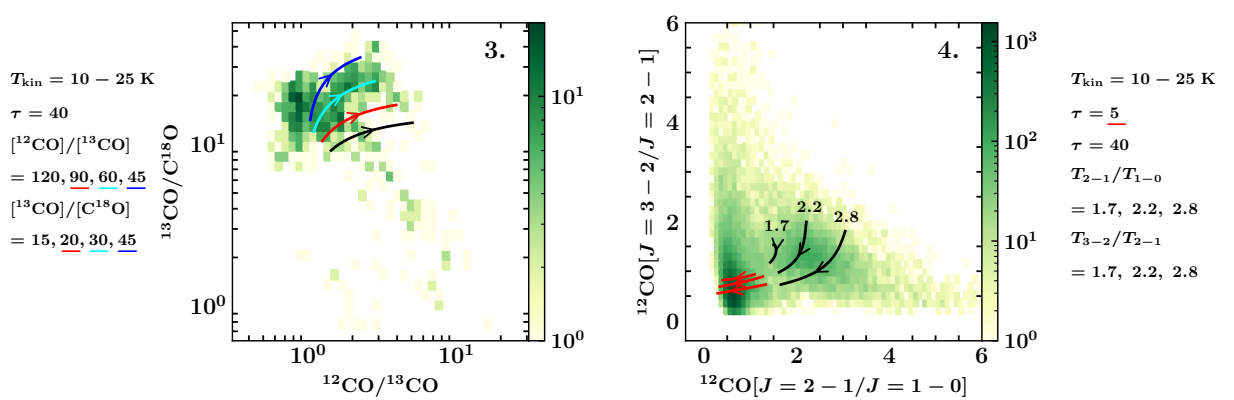

Fig. 9. Data histograms and LTE models of the emission of the rotational transitions of ${ }^{12} \mathrm{CO}$ and its isotopologs mapped in the extended $\mathrm{G}$ field with the IRAM $30 \mathrm{~m}$ and APEX: $1 .{ }^{13} \mathrm{CO}(1-0)$ vs. ${ }^{12} \mathrm{CO}(1-0) ; 2 .{ }^{13} \mathrm{CO}(1-0)$ vs. $\mathrm{C}^{18} \mathrm{O}(1-0) ; 3$. $\left[{ }^{13} \mathrm{CO}(1-0)\right] /\left[\mathrm{C}^{18} \mathrm{O}(1-0)\right]$ vs. $\left[{ }^{12} \mathrm{CO}(1-0)\right]$ $/\left[{ }^{13} \mathrm{CO}(1-0)\right]$; and $4 .{ }^{12} \mathrm{CO}(3-2) /{ }^{12} \mathrm{CO}(2-1)$ vs. ${ }^{12} \mathrm{CO}(2-1) /{ }^{12} \mathrm{CO}(1-0)$. The color map corresponds to the amount of data points (line of sight and velocity channels) that fall into a given bin of the histogram. The curves represent families of models of the LTE intensity as a function of the kinetic temperature of ${ }^{13} \mathrm{CO}$. The arrows indicate the direction in which the kinetic temperature grows along a given curve. Control parameters of the LTE models are given in Table 4 for each histogram. The dashed black lines represent the $3 \sigma$ detection level for each axis (histograms 1 and 2).

The ${ }^{13} \mathrm{CO}(1-0)$ vs. $\mathrm{C}^{18} \mathrm{O}(1-0)$ histogram has a much smaller number of bins determined with a good $(\mathrm{S} / \mathrm{N})(n=833)$ due to the faint emission of $\mathrm{C}^{18} \mathrm{O}(1-0)$ that is hardly detected at a $3 \sigma$ confidence level within our data cube. Still, we find evidence of at least one statistically significant branch. As a result of to the small size of the sample, it is not possible to identify any spatial or spectral correlation with certainty.
The third histogram has a poor statistical sample for the same reason as the second one $(n=833)$. The $\left[{ }^{13} \mathrm{CO}(1-0)\right] /$ $\left[\mathrm{C}^{18} \mathrm{O}(1-0)\right]$ ratio vs. $\left[{ }^{12} \mathrm{CO}(1-0)\right] /\left[{ }^{13} \mathrm{CO}(1-0)\right]$ relationship is localized in an area with little dispersion. Hence, for high signalto-noise measurements the isotopic ratios are almost uniform in the field of observations. Nonetheless, the lower signal-tonoise data bins display a statistically well-defined comet-shaped 
Table 4. Control parameters of the LTE models represented in Fig. 9.

\begin{tabular}{ccccccc}
\hline \hline Histogram & $\tau_{12} \mathrm{CO}(20 \mathrm{~K})$ & $T_{\text {kin }}(\mathrm{K})$ & {$\left[{ }^{12} \mathrm{CO} /{ }^{13} \mathrm{CO}\right]$} & {$\left[{ }^{13} \mathrm{CO} / \mathrm{C}^{18} \mathrm{O}\right]$} & $T_{{ }^{12} \mathrm{CO}} / T_{13} \mathrm{CO}$ & $T_{13 \mathrm{CO}} / T_{\mathrm{C}{ }^{18} \mathrm{O}}$ \\
\hline $1 .{ }^{13} \mathrm{CO}(1-0)$ vs. ${ }^{12} \mathrm{CO}(1-0)$ & {$[5,10,20,30,50,75]$} & {$[10-25]$} & 60 & 30 & 1 & 1 \\
$2 .{ }^{13} \mathrm{CO}(1-0)$ vs. ${ }^{18} \mathrm{CO}(1-0)$ & {$[5,10,20,30,50,75]$} & {$[10-25]$} & 60 & 30 & 1 & 1 \\
$3 .\left[{ }^{13} \mathrm{CO} /{ }^{18} \mathrm{CO}\right]$ vs. $\left[{ }^{12} \mathrm{CO} /{ }^{13} \mathrm{CO}\right]$ & 40 & {$[10-25]$} & {$[120,90,60,45]$} & {$[15,20,30,45]$} & 1 & 1 \\
\hline Histogram & $\tau_{12} \mathrm{CO}(20 \mathrm{~K})$ & $T_{\text {kin }}(\mathrm{K})$ & $T_{2-1} / T_{1-0}$ & $T_{3-2} / T_{2-1}$ & $\tau_{2-1} / \tau_{1-0}$ & $\tau_{3-2} / \tau_{2-1}$ \\
\hline $4 .{ }^{12} \mathrm{CO}[3-2 / 2-1]$ vs. $[2-1 / 1-0]$ & {$[5,40]$} & {$[10-25]$} & {$[1.7,2.2,2.8]$} & {$[1.7,2.2,2.8]$} & 0.2 & 1 \\
\hline
\end{tabular}

Notes. $\tau_{12} \mathrm{CO}$ is the list of optical depths of the lines used to generate the family of curves, $T_{13} \mathrm{CO}$ and $T_{12} \mathrm{CO}$ are the ranges of kinetic temperature used for ${ }^{13} \mathrm{CO}$ and ${ }^{12} \mathrm{CO}$, respectively. $\left[{ }^{12} \mathrm{CO} /{ }^{13} \mathrm{CO}\right]$ and $\left[{ }^{13} \mathrm{CO} / \mathrm{C}^{18} \mathrm{O}\right]$ are the adopted isotopic ratios. The ratios of excitation temperatures for different isotopologs or rotational transitions are indicated by the columns $T_{a} / T_{b}$, and the ratios of opacities for distinct rotational transitions are given in the columns $\tau_{a} / \tau_{b}$. The colors used for control parameters in this table correspond to the colors of the curves in Fig. 4.

branch extending from this area. This branch might correspond to distinct physical conditions and/or isotopic ratios for a fraction of the field of observations. The high $(\mathrm{S} / \mathrm{N})$ area of this branch is spatially correlated with the cloudlet and ring-like structure, whereas the "tail" of the branch is spatially and spectrally associated with the shocked clump.

The fourth histogram is built on a large statistical sample with high signal-to-noise data $(n=9793)$, as the rotational lines $J=1-0, J=2-1$, and $J=3-2$ are well detected in our data cubes. The ${ }^{12} \mathrm{CO}[3-2] /[2-1]$ vs. [2-1]/[1-0] relationship is clearly bimodal. A quasi-vertical branch centered on $[2-1] /[1-0] \simeq 0.5$ can be distinguished from a crescent-shaped branch centered on $[2-1] /[1-0] \simeq 2$ and $[3-2] /[2-1] \simeq 1.3$. The crescent-shaped branch is highly correlated to the high-velocity wings of the ${ }^{12} \mathrm{CO}$ lines tracing the emission of shocked gas, where it is expected to measure high, enhanced $J=2-1 / J=1-0$ ratio (e.g., Seta et al. 1998).

We compared the observational histograms with synthetic families of curves generated using modified LTE radiative transfer models of the observed rotational transition for ${ }^{12} \mathrm{CO},{ }^{13} \mathrm{CO}$, and $\mathrm{C}^{18} \mathrm{O}$. Assuming that $\mathrm{CO}$ is at thermal equilibrium, the intensity of the line integrated over an element of spectral resolution $\left(\Delta v=0.5 \mathrm{~km} \mathrm{~s}^{-1}\right)$ is given by

$W_{\alpha}=T_{\alpha} \Delta v$

where $\alpha=12,13,18$ (histograms 1-3) or $\alpha=1-0,2-1,3-2$ (histogram 4) for the ${ }^{12} \mathrm{CO},{ }^{13} \mathrm{CO}, \mathrm{C}^{18} \mathrm{O}$ isotopologs, respectively, and $J=1-0, J=2-1, J=3-2$ rotational lines. The quantity $T_{\alpha}$ is the integrated intensity of the line, defined as

$T_{\alpha}=T_{\alpha}^{0}\left(1-\mathrm{e}^{-\tau_{\alpha}}\right) \cdot\left(\frac{1}{\mathrm{e}^{\left(T_{\alpha}^{0} / T_{\alpha}^{\mathrm{exc}}\right)}-1}-\frac{1}{\mathrm{e}^{\left(T_{\alpha}^{0} / T_{\mathrm{cmb}}\right)}-1}\right)$,

where $T_{\alpha}^{0}=h v / k_{\mathrm{B}}$ is the temperature of the transition or energy of the upper level $E_{\text {up }}$, given in Table 1 for all studied lines; $\tau_{\alpha}$ is the optical depth of the line; $T_{\mathrm{cmb}}$ is the cosmic microwave background temperature; and $T_{\alpha}^{\mathrm{exc}}$ is the excitation temperature of the line. The optical depth $\tau_{\alpha}$ and its dependence on the excitation temperature is described by

$\tau_{\alpha}=\tau_{\alpha}^{T_{0}}\left(\frac{T_{0}}{T_{\alpha}^{\mathrm{exc}}}\right)\left(\frac{1-\mathrm{e}^{\left(T_{\alpha}^{0} / T_{\alpha}^{\mathrm{exc}}\right)}}{1-\mathrm{e}^{\left(T_{\alpha}^{0} / T_{0}\right)}}\right)$,

where $\tau_{\alpha}^{T_{0}}$ is the optical depth at a kinetic temperature $T_{0}$. We used the optical depth at $T_{0}=20 \mathrm{~K}$ as a reference. We made the assumption that the excitation temperature of the different isotopologs $\left(T_{\mathrm{exc}}^{12}, T_{\mathrm{exc}}^{13}, T_{\mathrm{exc}}^{18}\right)$ and rotational lines $\left(T_{\mathrm{exc}}^{1-0}, T_{\mathrm{exc}}^{2-1}\right.$, $T_{\text {exc }}^{3-2}$ ) are distinct and controlled their ratios as four supplementary independent parameters of the modified LTE models (Roueff et al. 2020). The opacities of ${ }^{12} \mathrm{CO}$ and $\mathrm{C}^{18} \mathrm{O}$ isotopologs were determined from the optical depth of ${ }^{13} \mathrm{CO}$ using the corresponding isotopic ratio as follows:

$\frac{\tau_{12}}{\tau_{13}}=\frac{\left[{ }^{12} \mathrm{CO}\right]}{\left[{ }^{13} \mathrm{CO}\right]}, \frac{\tau_{13}}{\tau_{18}}=\frac{\left[{ }^{13} \mathrm{CO}\right]}{\left[\mathrm{C}^{18} \mathrm{O}\right]}$

Lastly, we assumed a hierarchy in the optical depth of the different rotational lines such that $\tau_{1-0} \geq \tau_{2-1} \geq \tau_{3-2}$ and control their ratio as two supplementary parameters of the modified LTE models (Roueff et al. 2020). Hence, there are a total of nine control parameters that we can set: the optical depth $\tau_{13}$, isotopic ratio $\left[{ }^{12} \mathrm{CO}\right] /\left[{ }^{13} \mathrm{CO}\right]$, isotopic ratio $\left[{ }^{13} \mathrm{CO}\right] /\left[\mathrm{C}^{18} \mathrm{O}\right]$, temperature ratios $\left[T_{{ }^{12} \mathrm{CO}} / T_{{ }^{13} \mathrm{CO}}\right],\left[T_{{ }^{13} \mathrm{CO}} / T_{\mathrm{C}^{18} \mathrm{O}}\right],\left[T_{3-2} / T_{2-1}\right],\left[T_{2-1} / T_{1-0}\right]$, and optical depth ratios $\left[\tau_{3-2} / \tau_{2-1}\right],\left[\tau_{2-1} / \tau_{1-0}\right]$. We used the kinetic temperature of ${ }^{13} \mathrm{CO}$ as a variable of the parametric equation $I_{i j}\left(T_{\text {kin }}^{13}\right)$ to synthesize LTE models that we can plot on top of each 2D data histogram. Each curve in Fig. 9 corresponds to a given set of control parameters, with the kinetic temperature growing linearly along a curve. We produced a family of LTE models with linearly increasing optical depth and a kinetic temperature varying between 10 and $25 \mathrm{~K}$, corresponding to the typical temperatures of cold molecular clouds. We adopted the isotopic ratio $\left[{ }^{12} \mathrm{CO}\right] /\left[{ }^{13} \mathrm{CO}\right]$ and $\left[{ }^{13} \mathrm{CO}\right] /\left[\mathrm{C}^{18} \mathrm{O}\right]$ that offers the best visual match with our data, and similarly fine-tuned the other control parameters to reproduce the branches observed in each $2 \mathrm{D}$ data histogram.

All other parameters remaining constants, each control parameter has an effect on the pattern and location of a curve in the histogram space:

- An increase in the isotopic ratio $\left[{ }^{12} \mathrm{CO}\right] /\left[{ }^{13} \mathrm{CO}\right]$ translates the curves downward in the histogram 1, does not modify the histogram 2, and translates the curves rightward in the histogram 3.

- An increase in the isotopic ratio $\left[{ }^{13} \mathrm{CO}\right] /\left[\mathrm{C}^{18} \mathrm{O}\right]$ does not modify the histogram 1, translates the curves upward in the histogram 2, and translates the curves upward in the histogram 3.

- The modification of the temperature ratios alters the shape and orientation of the curves in histograms 3 and 4, and determines the boundaries of the ${ }^{12} \mathrm{CO}$ and $\mathrm{C}^{18} \mathrm{O}$ curves.

The LTE models that visually best match our data are represented in Fig. 9 over the data histograms, and their corresponding set of control parameters are summarized in Table 4. A kinetic temperature in the range $10-25 \mathrm{~K}$ is sufficient to account for most of the data where the sample is statistically significant, and we 
did not need to assume that $\left[T_{{ }^{12} \mathrm{CO}} / T_{{ }^{13} \mathrm{CO}}\right]$ and $\left[T_{{ }^{13} \mathrm{CO}} / T_{\mathrm{C}^{18} \mathrm{O}}\right]$ are different from 1 to find a satisfactory fit. However, to obtain a model that accounts for the high $[3-2] /[2-1]$ and $[2-1] /[1-0]$ data bins (histogram 4), it was necessary to set their temperature ratios as greater than 1 , such that we inferred an average temperature of up to $\sim 55 \mathrm{~K}$ traced by the $J=2-1$ lines, and up to $\sim 120 \mathrm{~K}$ for the $J=3-2$ lines in the high-velocity wings. This area of the histogram 4 also required lower $\tau_{2-1} / \tau_{1-0}$ and $\tau_{3-2} / \tau_{1-0}$ ratios, that we set to 0.2 for our models, suggesting that optical depth decreases significantly in the high-velocity wings of the lines. Considering histogram 3, our best models indicate a value of the isotopic ratio $\left[{ }^{12} \mathrm{CO}\right] /\left[{ }^{13} \mathrm{CO}\right]=45-90$ and $\left[{ }^{13} \mathrm{CO}\right] /\left[\mathrm{C}^{18} \mathrm{O}\right]=20-45$ to account for the emission of the different isotopologs observed in the extended $\mathrm{G}$ field. A large range of isotopic ratios can account for the observed data spread in histogram 3 , hence this result does not provide a precise determination of the $\left[{ }^{12} \mathrm{CO}\right] /\left[{ }^{13} \mathrm{CO}\right]$ and $\left[{ }^{13} \mathrm{CO}\right] /\left[\mathrm{C}^{18} \mathrm{O}\right]$ abundances. The higher boundary of our ${ }^{12} \mathrm{C} /{ }^{13} \mathrm{C}$ ratio estimate is anomalous with respect to the local ISM average of $62 \pm 4$ (Langer \& Penzias 1993), however Wilson \& Rood (1994) provide an estimate of the elemental abundances based on their distance to the Galactic center (GC):

$$
\begin{aligned}
& { }^{12} \mathrm{C} /{ }^{13} \mathrm{C}=(7.5 \pm 1.9) D_{\mathrm{GC}}+(7.6 \pm 12.9) \\
& { }^{16} \mathrm{O} /{ }^{18} \mathrm{O}=(58.8 \pm 11.8) D_{\mathrm{GC}}+(37.1 \pm 82.6) .
\end{aligned}
$$

If we adopt the distance of $1.9 \mathrm{kpc}$ for IC443 (Ambrocio-Cruz et al. 2017) the SNR is located at a distance of $\sim 10 \mathrm{kpc}$ from the GC. Hence our estimate of the ${ }^{12} \mathrm{CO} /{ }^{13} \mathrm{CO}$ ratio is in agreement with the expected abundance of $80 \pm 30$, but our measured ${ }^{13} \mathrm{CO} / \mathrm{C}^{18} \mathrm{O}$ is much higher than the predicted ratio of $8 \pm 2$. The measured enhancement of the ${ }^{13} \mathrm{CO} / \mathrm{C}^{18} \mathrm{O}$ ratio might be the product of the fractionation of carbon monoxide by photodissociation, as the shielding of the various isotopologs of $\mathrm{CO}$ provides a mechanism for the rarefaction of $\mathrm{C}^{18} \mathrm{O}$ with respect to the expected abundance (e.g., Glassgold et al. 1985). We checked the spatial distribution of data points corresponding to particular areas of the histograms presented in Fig. 9.

- Histogram 3: interestingly, the data points corresponding to high $(>20){ }^{13} \mathrm{CO} / \mathrm{C}^{18} \mathrm{O}$ line ratio are spatially correlated with the cloudlet only. If no protostars are positively identified toward the cloudlet (Sect. 5), radiative decay from X-ray irradiation seen in the SNR cavity with XMM-Newton might provide a source to account for the selective photodissociation of $\mathrm{CO}$ within the cloudlet (Troja et al. 2006; Troja et al. 2008).

- Histogram 4: data points corresponding to high $(>2)$ $[J=2-1] /[J=1-0]$ and $[J=3-2] /[J=2-1]$ ratios are spatially correlated with the shocked clump, and spectrally correlated with the high-velocity wings of the molecular line. The most striking spatial correlation is seen toward the eastern surface layer of the shocked clump, where the molecular gas seems to be systematically characterized by a large $(>2)[J=3-2] /[J=2-1]$ and small $(<1)[J=2-1] /[J=1-0]$ ratio apparently tracing the shock front. However, this combination of line ratios is not predicted by RADEX models and could be due to an inaccurate registration between the data sets.

\subsubsection{Population diagrams}

We determined the physical conditions (column density $N_{\mathrm{CO}}$, and kinetic temperature $T_{\text {kin }}$ ) of the molecular gas in the extended $\mathrm{G}$ region assuming that the emission lines are thermalized. We used pixel-per-pixel population diagrams (Goldsmith \& Langer 1999) corrected for optical depth for the ${ }^{12} \mathrm{CO}(3-2)$,
${ }^{12} \mathrm{CO}(2-1)$, and ${ }^{12} \mathrm{CO}(1-0)$ transitions to measure the upper level column density $N_{\text {up }}$ (hereafter level population) for $J_{\text {up }}=3$, $J_{\text {up }}=2, J_{\text {up }}=1$. Systematically, the ${ }^{13} \mathrm{CO}$ data is used to correct the effect of optical depth in the population diagrams, for each element of resolution (i.e., for each of line of sight), and for all velocity channels. To perform this task we chose to use the APEX data cube over the IRAM $30 \mathrm{~m}$ data cube to measure the specific intensity corresponding to the ${ }^{12} \mathrm{CO}(2-1)$ transition. The IRAM $30 \mathrm{~m}$ has the advantage of having lower noise (see Table 2). However, the ${ }^{13} \mathrm{CO}(2-1)$ transition was only observed with the APEX telescope owing to receiver setup constraints. Hence to avoid intercalibration issues, we used the APEX data for ${ }^{12} \mathrm{CO}(2-1)$ and ${ }^{13} \mathrm{CO}(2-1)$. First, we convolved all maps to the same spatial resolution for consistency, using the nominal resolution of ${ }^{13} \mathrm{CO}(2-1)$, such that we have a beam diameter of $30.1^{\prime \prime}$ for the six maps considered. The spectral resolution was also modified and set to $2 \mathrm{~km} \mathrm{~s}^{-1}$ for each transition to increase the $\mathrm{S} / \mathrm{N}$. Then, the following measurements were performed pixel-per-pixel using a Python ${ }^{5}$ algorithm:

1. Sigma-clipping was applied to the spectra of all transitions of the two isotopologs ${ }^{12} \mathrm{CO}$ and ${ }^{13} \mathrm{CO}$, using a threshold of $3 \sigma$.

2 . In every single velocity channel remaining after sigmaclipping, the $N_{J=3}, N_{J=2}$, and $N_{J=1}$ level populations were measured both for ${ }^{12} \mathrm{CO}$ and ${ }^{13} \mathrm{CO}$ to determine the optical depth of the three transitions using the adopted value of isotopic ratio. We measured the optical depths $\tau_{3-2}, \tau_{2-1}$ and $\tau_{1-0}$, assuming that ${ }^{13} \mathrm{CO}$ lines are optically thin and that the excitation temperatures of the two isotopologs are approximately equal (this second assumption is supported by the results presented in Sect. 9). Under this assumption we have

$$
\begin{aligned}
& \int I_{v,{ }^{12} \mathrm{CO}} \mathrm{d} v=\frac{h v_{i j,{ }^{12} \mathrm{CO}}}{4 \pi} N_{\mathrm{up},{ }^{12} \mathrm{CO}} A_{i j,{ }^{12} \mathrm{CO}} \cdot \beta_{i j} \\
& \int I_{v,{ }^{13} \mathrm{CO}} \mathrm{d} v=\frac{h v_{i j,{ }^{13} \mathrm{CO}}}{4 \pi} N_{\mathrm{up},{ }^{13} \mathrm{CO}} A_{i j,{ }^{13} \mathrm{CO}}
\end{aligned}
$$

where $\beta_{i j}=\left[1-\mathrm{e}^{-\tau_{i j}}\right] / \tau_{i j}$ is the escape probability of a photon, $v_{i j}$ the frequency of the transition, $N_{\text {up }}$ the level population, $A_{i j}$ the probability of the transition, and $I_{v}$ the specific intensity. Based on the comparison of our data with modified LTE models (Sect. 4.2.1) we adopted different values for the expected isotopic ratio toward each region (indicated in Table 5).

3. The estimates of ${ }^{12} \mathrm{CO}$ level populations were corrected for optical depth using the correction factor $C_{\tau_{i j}}=\tau_{i j} /\left(1-e^{-\tau_{i j}}\right)$, such that the corrected column density is given by $N_{\text {up }}^{*}=C_{\tau_{i j}} N_{\text {up }}$.

4. We put the corrected level populations $N_{\text {up }}^{*}$ and their corresponding energies $E_{\mathrm{up}}=h v_{i j} / k_{\mathrm{B}}$ into the population diagram $N_{\text {up }}^{*}=f\left(E_{\text {up }}\right)$ and used a $\chi^{2}$-minimization algorithm ${ }^{6}$ to determine the best linear fit $y=a x+b$. The excitation temperature $T_{\text {ex }}$ is deduced from the slope, and the total column density $N_{\mathrm{CO}}$ is determined by computing the partition function $Q\left(T_{\mathrm{ex}}\right)^{7}$ and measuring the offset.

5. We finally derived the mass of the gas from the measured total column density $N_{\mathrm{CO}}$, using the expected $\left[\mathrm{H}_{2}\right] /\left[{ }^{12} \mathrm{CO}\right]$

5 Python Core Team (2015). Python: a dynamic, open source programming language. Python Software Foundation. https://www . python. org/

6 The Python module scipy.optimize.curve_fit uses the LevenbergMarquardt least-squares curve fitting algorithm.

7 We computed the partition function taking into account the first 40 upper rotational levels of ${ }^{12} \mathrm{CO}$ taken from the Cologne Database for Molecular Spectroscopy and Jet Propulsion Laboratory database. The thermal energy of the 40th level is $E_{40}=4513 \mathrm{~K}$. 
Table 5. Results of the population diagrams analysis for each structure.

\begin{tabular}{cccccccc}
\hline \hline Region & $\overline{N_{\mathrm{CO}}}\left(10^{17} \mathrm{~cm}^{-2}\right)$ & Mass $\left(M_{\odot}\right)$ & $\frac{{ }^{12} \mathrm{CO}}{13 \mathrm{CO}}$ & $\overline{T_{\mathrm{exc}}}(\mathrm{K})$ & $\log _{10}\left(\chi^{2}\right)$ & $\overline{T_{\mathrm{exc}}^{\mathrm{W}}}(\mathrm{K})$ & $M_{X_{\mathrm{CO}}}^{J=1-0}\left(M_{\odot}\right)$ \\
\hline Cloudlet (A) & $1.4-2.3(3.7)$ & $130-210(330)$ & $80 \pm 20$ & $9_{-3}^{+1}$ & 0.34 & $12 \pm 1$ & $450 \pm 140$ \\
Ring-like structure (B) & $2.3-3.8(4.4)$ & $60-110(130)$ & $60 \pm 20$ & $11_{-2}^{+1}$ & -0.68 & $13 \pm 1$ & $200 \pm 60$ \\
Shocked clump (C) (i) & $0.21-0.32(0.40)$ & $12-17(22)$ & $45 \pm 10$ & $28 \pm 2$ & -0.85 & $32 \pm 2$ & $190 \pm 60$ \\
Shocked clump (C) (ii) & $1.6-2.8(3.4)$ & $80-150(230)$ & $45 \pm 10$ & $12_{-5}^{+1}$ & -0.18 & $19 \pm 2$ & $240 \pm 70$ \\
Shocked clump (C) (iii) & $0.12-0.14(0.23)$ & $6-7(12)$ & $45 \pm 10$ & $22 \pm 2$ & -0.08 & $28 \pm 2$ & $110 \pm 40$ \\
Shocked knot (D) & $0.19-0.24$ & $2-4(6)$ & $45 \pm 10$ & $21 \pm 2$ & -0.38 & $30 \pm 2$ & $30 \pm 10$ \\
Ambient cloud & $1.1-1.9(3.0)$ & $400-700(1800)$ & $60 \pm 20$ & $9_{-5}^{+1}$ & 0.85 & $10 \pm 1$ & $1300 \pm 400$ \\
Foreground clump & $0.02-0.03(0.07)$ & $3-5(14)$ & $45 \pm 10$ & $9 \pm 1$ & 0.07 & $11 \pm 1$ & $38 \pm 11$ \\
\hline IC443G (extended) & $1.6-2.8(4.0)$ & $900-1600(3100)$ & - & $7 \pm 1 / 9 \pm 1$ & 0.89 & $11 \pm 1$ & $2600 \pm 800$ \\
\hline
\end{tabular}

Notes. The value $\overline{N_{\mathrm{CO}}}$ is the average column density and $M_{\odot}$ the mass in solar unit. The adopted isotopic ratio ${ }^{12} \mathrm{CO} /{ }^{13} \mathrm{CO}$ for each region is indicated in the fourth column. In the mass column, we indicate in parenthesis the "cold mass" estimated by considering only the upper levels $J=1,2$. The value $\overline{T_{\mathrm{exc}}}$ is the average excitation temperature. The quality of the linear fits is indicated by the average value of $\chi^{2}$. The value $\overline{T_{\mathrm{exc}}^{\mathrm{W}}}$ is the average excitation temperature of the warm component, obtained by considering the levels $J=2,3$ separately from the level $J=1$. For comparison, we indicate the estimate of the mass obtained using the CO-to- $\mathrm{H}_{2}$ conversion factor $X_{\mathrm{CO}}=2 \times 10^{20} \mathrm{~cm}^{-2}\left(\mathrm{~K} \mathrm{~km} \mathrm{~s}^{-1}\right)^{-1}$ applied to the raw $J=1-0$ data (without optical depth correction).

Table 6. Velocity ranges $\left[v_{\min }, v_{\max }\right]$ and spatial boxes (as defined in Fig. 6) used to measure specific intensity corresponding to each structure.

\begin{tabular}{ccc}
\hline \hline Region & {$\left[v_{\min }, v_{\max }\right]\left(\mathrm{km} \mathrm{s}^{-1}\right)$} & Box (Fig. 6) \\
\hline Cloudlet & {$[-8.5 ;-4.5]$} & $\mathrm{A}$ \\
Ring-like structure & {$[-7.0 ;-4.5]$} & $\mathrm{B}$ \\
Shocked clump $i$ & {$[-35 ;-6.5]$} & $\mathrm{C}$ \\
Shocked clump $i i$ & {$[-6.5 ;-1.5]$} & $\mathrm{C}$ \\
Shocked clump $i i i$ & {$[-1.5 ;+25]$} & $\mathrm{C}$ \\
Shocked knot & {$[-35 ;+25]$} & $\mathrm{D}$ \\
Ambient cloud & {$[-6.5 ;-1.5]$} & $\overline{\mathrm{A}} \cap \overline{\mathrm{B}} \cap \overline{\mathrm{C}} \cap \overline{\mathrm{D}}$ \\
\hline
\end{tabular}

Notes. Three velocity ranges are given for the shocked clump, as we distinguish three components within the molecular lines: (i) the left (blueshifted) high-velocity wing, (ii) the core, and (iii) the right (redshifted) high-velocity wing.

ratio toward dense molecular regions and assuming that the distance of IC443 is $1.9 \mathrm{kpc}$ (Ambrocio-Cruz et al. 2017). We adopted the value of $10^{4}$ for the $\mathrm{H}_{2}$-to- ${ }^{12} \mathrm{CO}$ abundance ratio (Gordon \& Burton 1976; Frerking et al. 1982), thus we have $M_{\mathrm{H}_{2}}=\left(2 m_{\mathrm{H}}\right) \mathcal{A} N_{\mathrm{CO}} \times 10^{4}$, where $\mathcal{A}$ is the area of the box and $m_{\mathrm{H}}$ the mass of hydrogen.

Uncertainties. The error bars on total CO column density, gas mass, and kinetic temperature are determined by the following: (i) instrumental errors, dominated by the systematic uncertainty on the flux (Sect. 3.1.3); (ii) the quality of the linear fit applied to the population diagram; and (iii) systematic errors on the adopted distance of IC443 and the ${ }^{12} \mathrm{CO} /{ }^{13} \mathrm{CO}$ isotopic ratio.

Results. Average population diagrams are presented in Figs. 10 and 11, based on the spatial boxes defined in Fig. 6 and integration ranges defined in Table 6 . The measurements obtained are presented in Table 5, in which the minimum and maximum boundaries are given for the total CO column density, gas mass, and kinetic temperature. We measured the column densities and masses for the regions A (cloudlet), B (ring-like structure), C (shocked clump), and D (shocked knot) as indicated in Fig. 6, and also for the ambient cloud by averaging the signal over the entire field (regions A, B, C and D excluded). We also measured the mass of the foreground clump that spatially coincides with the shocked clump using a Gaussian model for the average $\mathrm{CO}$ lines in the velocity range $[2.5 ; 12] \mathrm{km} \mathrm{s}^{-1}$. The result of this measurement is presented at the end of Table 5.

\subsubsection{Discussion}

As a first-order verification we compared our measurement of the mass based on the population diagram with a rough estimate of the mass using the $\mathrm{CO}(J=1-0)$-to- $\mathrm{H}_{2}$ conversion factor $X_{\mathrm{CO}}$ (Dame et al. 2001; Bolatto et al. 2013). We adopted the following conversion factor, with a $\pm 30 \%$ uncertainty, which is written as

$X_{\mathrm{CO}}=2 \times 10^{20} \mathrm{~cm}^{-2}\left(\mathrm{~K} \mathrm{~km} \mathrm{~s}^{-1}\right)^{-1}$.

The $\mathrm{H}_{2}$ column density was determined by the product of $X_{\mathrm{CO}}$ with the area of the raw ${ }^{12} \mathrm{CO} J=1-0$ line (without optical depth correction), and the mass was inferred from $N_{\mathrm{H}_{2}}$ in the same manner as described previously. We report the results of this measurement in the last column of Table 5, referred to as $M_{X_{\mathrm{CO}}}^{J=1-0}$. Within error bars, almost all our measurements are consistent with this rough estimate of the mass, except for the line wings toward the shocked clump. With respect to the population diagram method, this method systematically overestimates the mass. The enhanced $J=2-1 / J=1-0$ emission of the highvelocity gas accounts for a lower mass in the population diagram, as it traces a warmer gas for which the $X_{\mathrm{CO}}$ conversion factor yields an overestimate.

We built a single population diagram for each spatial box, using a single value of $N_{\text {up }}$ that is the spatial and spectral average of our sample of measurements. The resulting population diagrams are shown in Fig. 10; statistical information on the spread of the sample around these average values of $N_{\text {up }}$ is also represented. As additional information on the thermalization of carbon monoxide, in Fig. 11 we show the average population 

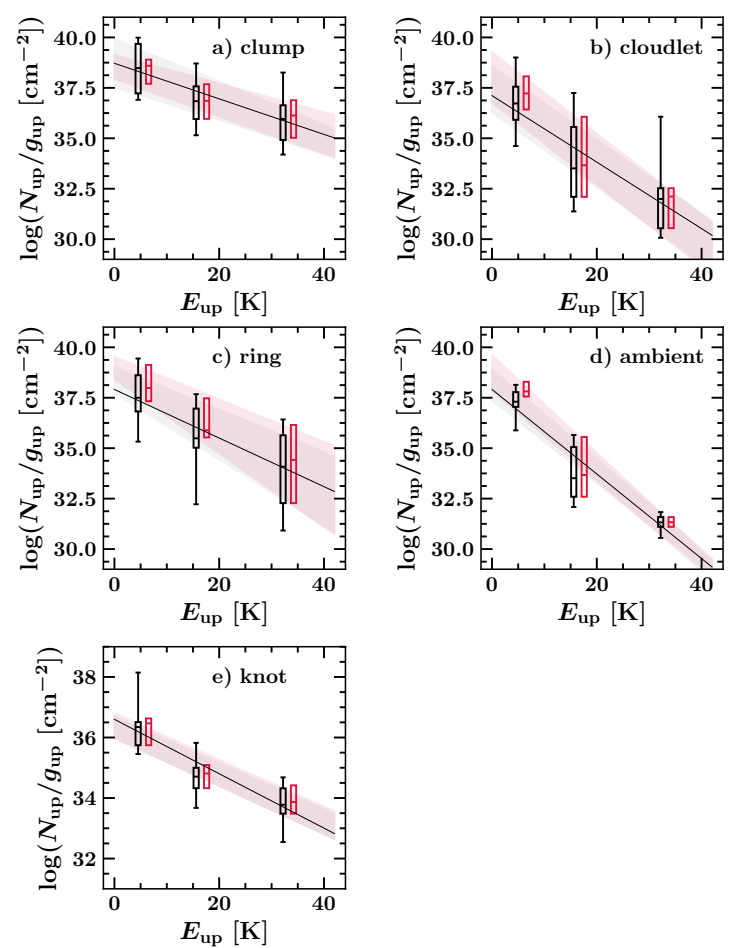

Fig. 10. Average population diagrams of ${ }^{12} \mathrm{CO}$ corrected for optical depth. The measured average, first quartile, third quartile, fifth percentile, and 95th percentile associated with our sample of level populations corresponding to $J_{\text {up }}=1, J_{\text {up }}=2$, and $J_{\text {up }}=3$ are indicated for two adopted values of the ${ }^{12} \mathrm{CO} /{ }^{13} \mathrm{CO}$ isotopic ratio $\left({ }^{12} \mathrm{CO} /{ }^{13} \mathrm{CO}=60\right.$ in black, ${ }^{12} \mathrm{CO} /{ }^{13} \mathrm{CO}=100$ in red). The black solid line represents the linear fit of the black data points. The boundaries of the filled areas are defined by the linear fits corresponding to the first and third quartiles for each value of the isotopic ratio adopted. The 5 th percentile and 95 th percentile are indicated by the caps, the first quartile and third quartile are indicated by the ends of the box, and the average is indicated by the cap inside of the box. Each diagram accounts for a sample of measurements performed in one of the spatial boxes defined in Fig. 6 and integration ranges defined in Table 6: $(a)$ the shocked clump, $(b)$ the cloudlet, $(c)$ the ring, $(d)$ the ambient cloud, and $(e)$ the shocked knot.

diagrams obtained using the ${ }^{13} \mathrm{CO}$ lines with the same method, without correction of the optical depth.

1. Thermalization: at a temperature of $10 \mathrm{~K}$, the critical density $n_{i j}=A_{i j} / C_{i j}$ of the ${ }^{12} \mathrm{CO} J=1-0, J=2-1$ and $J=3-2$ lines are $n_{1-0}=2.2 \times 10^{3} \mathrm{~cm}^{-3}, n_{2-1}=2.3 \times 10^{4} \mathrm{~cm}^{-3}$, respectively, and $n_{3-2}=3.5 \times 10^{4} \mathrm{~cm}^{-38}$. Toward the shocked clump, it is likely that these critical densities are attained (Cesarsky et al. 1999), hence the lines should be thermalized. In the population diagrams shown in Fig. 10, the average data points are generally in satisfactory agreement with the assumption that the emission lines are thermalized, as there is no significant divergence from the Boltzmann distribution for any of the structure studied. The highest value of $\chi^{2}$ is obtained toward the ambient cloud, which also presents an abnormally low kinetic temperature, down to $\sim 5 \mathrm{~K}$. This might indicate that the $J=2-1$ and $J=3-2$ are sub-thermal, which is expected if some parts of the NW-SE molecular cloud we are probing have a density lower than $10^{4}$ $\mathrm{cm}^{-3}$. There is a systematic discrepancy between the linear fit and the measured column density of the upper level $J=2$, which

\footnotetext{
8 The Einstein coefficients $A_{i j}$ are given in Table 1, and the collisional excitation rates $C_{i j}$ are taken from the Cologne Database for Molecular Spectroscopy and Jet Propulsion Laboratory database.
}
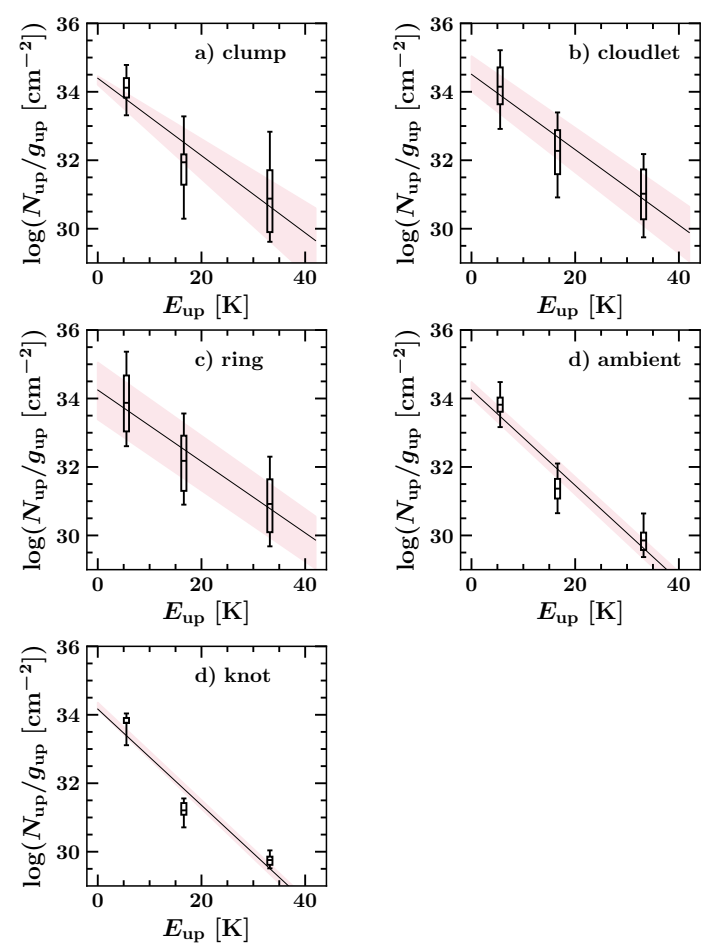

Fig. 11. Average population diagrams of ${ }^{13} \mathrm{CO}$. The measured average, first quartile, third quartile, 5th percentile, and 95th percentile associated with our sample of measured level populations are indicated for each level. The black solid line represents the linear fit of the average data points. The boundaries of the filled areas are defined by the linear fits corresponding to the first and third quartiles. Each diagram accounts for a sample of measurements performed in one of the spatial boxes, as in Fig. 10.

is lower than expected in each structure. This anomaly can be solved if we adopt two distinct kinetic temperatures to model the relative distribution of the level populations. In the highvelocity wings of the lines, it is expected and it was hinted by our LTE analysis (Sect. 4.2.1) that the $J=2-1$ and $J=3-2$ lines trace a warmer gas than the $J=1-0$ line that is primarily tracing the quiescent and cold phase. In this case, the distribution in these population diagrams should be modeled by a linear fit of kinetic temperature $T_{1}$ for the upper levels $J_{\text {up }}=1,2$ (cold component) and a linear fit of temperature $T_{2}$ for levels $J_{\text {up }}=2,3$ (warm component), hence assuming that along a line of sight we observe two layer of gas thermalized at distinct temperatures, where $T_{1}<T_{2}$. In Table 5 we present the temperature obtained for the warm component as well as the mass of the cold component. The statistical spread of our sample of measured level populations around the average linear distribution provides a strong motive for the use of pixel-per-pixel, channel-per-channel population diagrams, as it is evidence of a large range of physical conditions that require distinct LTE models.

2. Filling factor: the filling factor used to infer column densities from the main beam temperature measured during our observations is set to 1. From the morphology of the gas mapped in ${ }^{12} \mathrm{CO}(2-1)$ with the IRAM $30 \mathrm{~m}$ with a nominal resolution of $11.2^{\prime \prime}$ (Figs. 4, 2) we consider that we are probing extended, clumpy structures with dimensions that are greater than the beam diameter characterizing our observations, between and 19.2" and $30.1^{\prime \prime}$ for the data cubes used to measure the gas mass.

3. Optical depth: the measurement of the optical depth from the comparison of the fluxes between ${ }^{12} \mathrm{CO}$ and ${ }^{13} \mathrm{CO}$ is shown in the appendix along with the measurement based on 
the ${ }^{12} \mathrm{CO} / \mathrm{C}^{18} \mathrm{O}$ isotopic ratio (Fig. F.1). The optical depth is non-negligible both in the ring-like structure and cloudlet. It is important in the self-absorbed component of the emission lines corresponding to the shocked clump where the cold, ambient cloud intervenes, and much lower in the high-velocity wings. We checked the assumption that ${ }^{13} \mathrm{CO}$ is optically thin using the $\mathrm{C}^{18} \mathrm{O} J=1-0$ and $J=2-1$ lines to estimate its optical depth based on the expected ${ }^{13} \mathrm{CO} / \mathrm{C}^{18} \mathrm{O}$ isotopic ratio. In our pixel-per-pixel, channel-per-channel population diagrams the correction of the optical depth is partially biased, as the detection of ${ }^{13} \mathrm{CO}$ is limited by the noise level. At a certainty level of $5 \sigma$, we cannot measure a flux lower than 125,420 , and $400 \mathrm{mK}$ for the $J=1-0$, $J=2-1$, and $J=3-2$ transitions, respectively. As a consequence, toward a significant number of lines of sight and velocity channels the optical depth is measured for the $J=1-0$ transition but not for $J=2-1$ and $J=3-2$. In the population diagram this results in the biased displacement of the level $J=1$ with respect to the levels $J=2$ and $J=3$, hence the slope of the linear fit is artificially enhanced and the excitation temperature is lowered (Fig. 10, Table 5).

4. Self-absorption of CO lines toward the shocked clump: we performed an independent measure of the temperature and column density of the absorbing envelope of the shocked clump from the absorption features of $\mathrm{CO}$ lines. Toward the position $\left(6^{\mathrm{h}} 16^{\mathrm{m}} 42.5^{\mathrm{s}},+22^{\circ} 32^{\prime} 12^{\prime \prime}\right)$, we renormalized the signal in each frequency channel with respect to the profile of the emission lines and measured the optical depth for all transitions in a beam of radius $22.5^{\prime \prime}$. From the equivalent widths $W$ of the transitions $J=1-2$ and $J=0-1$, we measured the temperature of the gas solving the following equation:

$F\left(\frac{T_{0}}{T_{\mathrm{ex}}}\right)=\frac{W_{1 \rightarrow 2}}{4 W_{0 \rightarrow 1}}$,

where $T_{0}=h v / k$ and $F(u)=\mathrm{e}^{-3 u} \cosh (u)$ (see Appendix B). We obtained an excitation temperature of $18 \pm 3 \mathrm{~K}$. We then built a population diagram of the lower population $N_{l}$ levels from the measures of the optical depth, using the following relation:

$\tau=1.1 \times 10^{-15} f_{\mathrm{ul}}\left(\frac{N_{l}}{\mathrm{~cm}^{-2}}\right)\left(\frac{\lambda}{\AA}\right)\left(\frac{\sigma_{v}}{\mathrm{~km} \mathrm{~s}^{-1}}\right)$,

where $f_{\mathrm{ul}}$ is the oscillator force, $\lambda$ the wavelength of the transition, and $\sigma_{v}$ the velocity dispersion of the absorbing medium, which we assume to be equal to the velocity dispersion of the lines associated with the shocked clump that we measured in Table 3 . This population diagram yields a total column density of the absorbing medium $N_{\mathrm{CO}}=3.4 \times 10^{16} \mathrm{~cm}^{-2}$, corresponding to a total mass of $20 M_{\odot}$ for the absorbing envelope of the shocked clump.

\subsection{LVG method}

The results obtained in the previous section are based on several strong assumptions, in particular that the excitation temperature of ${ }^{12} \mathrm{CO}$ and ${ }^{13} \mathrm{CO}$ are equal. To address this issue, we investigated the application of a second method that does not require this assumption to determine the mass of the gas toward our field of observations using CO lines. We used the LVG model for an expanding spherical shell (e.g., Sobolev 1960, Surdej 1977) to model the lines observed. This model requires a local, LVG $\nabla v$ along the line of sight. We consider that this assumption is verified throughout the field of observation as the line width of ${ }^{12} \mathrm{CO}$ lines suggests typical turbulent broadening (Sect. 3.3).
We used the non-LTE radiative transfer RADEX code (van der Tak et al. 2007), a computer program that solves the radiative transfer equation based on the escape probability formulation in an isothermal and homogeneous medium characterized by a LVG. Three geometries are available. We selected the expanding spherical shell for which the escape probability is related to the optical depth $\tau$ by the following formula (Mihalas 1978):

$\beta_{\mathrm{LVG}}=\frac{1-\mathrm{e}^{-\tau}}{\tau}$

In the framework of the LVG assumption, the value of the optical depth at line center determined by RADEX is proportional to $N / \Delta v$, where $N$ is the column density and $\Delta v$ the full width at half-maximum of the line profile. Hence when using RADEX the choice of the parameter $\Delta v$ is critical to model the expected intensities of atomic and molecular lines. The program solves the statistical equilibrium equations taking into account up to seven collisional partners, allowing us to analyze spectral line observations if the molecular collisional data is known. RADEX models require a total of seven input parameters: the column density $N$, local density $n_{\mathrm{H}_{2}}$, kinetic temperature $T_{\text {kin }}$, line width $\Delta v$, and background temperature $T_{\mathrm{b}}$. In return, RADEX estimates the excitation temperature $T_{\text {ex }}$, optical depth $\tau_{i j}$, peak temperature $T_{\mathrm{R}}$, upper and lower level populations $N_{\text {up }}, N_{\text {low }}$, and flux $\int T \mathrm{~d} v$ of an arbitrary number of transitions defined by the user.

We applied the LVG method to average spectra measured over the extent of each box defined in Fig. 6 and velocity ranges defined in Table 6 . Using a $\chi^{2}$ minimization, we compared RADEX outputs with our measurements to constrain the physical conditions. We compared the line intensities of the three first rotational transitions of ${ }^{12} \mathrm{CO}$ and ${ }^{13} \mathrm{CO}$ with a grid of RADEX models. In the same manner as in the population diagram approach, for consistency we convolved all maps to the same spatial resolution, using the nominal resolution of ${ }^{13} \mathrm{CO}(2-$ 1 ), such that we have a beam diameter of $30.1^{\prime \prime}$ for the six maps considered. The spectral resolution was also set to $2 \mathrm{~km} \mathrm{~s}^{-1}$ for each transition to increase the $\mathrm{S} / \mathrm{N}$. Then we produced several 3D grids of RADEX models with the varying parameters $\left(N, n_{\mathrm{H}_{2}}\right.$, $T)$ and fixed parameters $\left(T_{\mathrm{b}}, \Delta v\right)$. These parameters are specified in Table 7 for the different grids used. We used large ranges of column densities and kinetic temperature to probe a consistent fraction of the space of parameters, corresponding to a variety of physical conditions as wide as possible. We set the value of the parameter $\Delta v$ based on the equivalent linewidth that we measured on average spectra using bi-Gaussian functions to model the line profiles. We took the following steps for each average spectrum using a Python algorithm:

1. We applied sigma-clipping to the spectra of all transitions of ${ }^{12} \mathrm{CO}$ and ${ }^{13} \mathrm{CO}$, using a threshold of $3 \sigma$.

2. We measured the specific intensity of each transition on the velocity range corresponding to each spectral feature (e.g., the wings of the shocked clump are treated separately from the core of the line). Then, in each element of the model grid we computed the following quantity, corresponding to a combined reduced $\chi^{2}$ statistical estimator:

$\chi_{\mathrm{RADEX}}^{2}=\frac{1}{2} \sum_{J_{\mathrm{up}}=1}^{3}\left[\frac{\left(s_{12}^{\mathrm{RADEX}}-s_{12}^{\mathrm{obs}}\right)^{2}}{3 \sigma\left(s_{12}^{\mathrm{obs}}\right)^{2}}+\frac{\left(s_{13}^{\mathrm{RADEX}}-s_{13}^{\mathrm{obs}}\right)^{2}}{3 \sigma\left(s_{13}^{\mathrm{obs}}\right)^{2}}\right]$.

In this equation, $J_{\text {up }}$ indicates the upper levels of each transition, $s_{12}^{\text {RADEX }}$ and $s_{13}^{\text {RADEX }}$ are respectively the specific intensity 
Table 7. Summary of the results and parameters used in the LVG analysis.

\begin{tabular}{lccccccc}
\hline \hline Regions & \multirow{2}{*}{ Cloudlet (A) } & \multirow{2}{*}{ Ring-like (B) } & \multicolumn{3}{c}{ Shocked clump (C) } & \multirow{2}{*}{ Shocked knot (D) } & Ambient \\
\cline { 3 - 6 } & & & Blueshifted & Core & Redshifted & & \\
\hline$N_{\mathrm{CO}}\left(10^{17} \mathrm{~cm}^{-2}\right)$ & $1.4-2.4$ & $1.7-2.3$ & $0.68-0.99$ & $0.9-1.48$ & $0.24-0.45$ & $0.17-0.22$ & $0.44-0.89$ \\
$T_{\text {kin }}(\mathrm{K})$ & $6 \pm 1$ & $10 \pm 1$ & $16 \pm 7$ & $12 \pm 2$ & $17 \pm 9$ & $13 \pm 6$ & $13 \pm 5$ \\
$n_{\mathrm{H}_{2}}\left(\mathrm{~cm}^{-3}\right)$ & $\sim 7 \times 10^{5}$ & $>10^{4}$ & $>10^{4}$ & $>10^{4}$ & $>10^{4}$ & $>10^{4}$ & $\sim 3 \times 10^{4}$ \\
$\log _{10}\left(\chi^{2}\right)$ & -0.04 & -0.16 & 0.23 & 0.04 & 0.48 & 0.12 & 0.47 \\
$M\left(M_{\odot}\right)$ & $120-220$ & $40-70$ & $32-52$ & $42-80$ & $19-26$ & $2-3$ & $210-350$ \\
$M_{\mathrm{L} V G} / M_{\mathrm{LTE}}$ & 1 & 0.64 & 2.90 & 0.53 & 3.46 & 0.83 & 0.51 \\
\hline$N_{12} \mathrm{CO}\left(\mathrm{cm}^{-2}\right)$ & {$\left[10^{15}-10^{19}\right]$} & {$\left[10^{15}-10^{19}\right]$} & {$\left[10^{15}-10^{19}\right]$} & {$\left[10^{15}-10^{19}\right]$} & {$\left[10^{15}-10^{19}\right]$} & {$\left[10^{15}-10^{19}\right]$} & {$\left[10^{15}-10^{19}\right]$} \\
$\left.{ }^{12} \mathrm{CO} /{ }^{13} \mathrm{CO}\right]$ & 80 & 60 & 45 & 45 & 45 & 45 & 60 \\
$T_{\text {grid }}(\mathrm{K})$ & {$[1-100]$} & {$[1-100]$} & {$[1-100]$} & {$[1-100]$} & {$[1-100]$} & {$[1-100]$} & {$[1-100]$} \\
$T_{\mathrm{b}}(\mathrm{K})$ & 2.7 & 2.7 & 2.7 & 2.7 & 2.7 & 2.7 & 2.7 \\
$\Delta v\left(\mathrm{~km} \mathrm{~s}^{-1}\right)$ & 4.0 & 3.5 & 25 & 5.0 & 25 & 35 & 3.0 \\
\hline
\end{tabular}

Notes. We show ranges of values corresponding to the uncertainty interval. $N_{\mathrm{CO}}$ is the column density of ${ }^{12} \mathrm{CO}, T_{\text {kin }}$ the kinetic temperature of the gas, and $n$ the local density of $\mathrm{H}_{2}$. The parameter $\log _{10}\left(\chi^{2}\right)$ gives the minimum value of $\chi^{2}$ within the minimization diagrams presented in Fig. 12 and $M$ is the mass measured using the $\left[\mathrm{H}_{2}\right] /\left[{ }^{12} \mathrm{CO}\right]$ ratio. The parameter $N_{\text {grid }}$ is the range of column densities parsed across the grid of models, $T_{\text {grid }}$ the range of kinetic temperatures, $T_{\mathrm{b}}$ and $\Delta v$ are the background temperature and width of the emission line used by RADEX for all models, respectively. The ratio $M_{\mathrm{LVG}} / M_{\mathrm{LTE}}$ of the estimate of the mass with the LVG approach is indicated with respect to the results obtained with population diagrams (Sect. 4.2.2, Table 5).

of ${ }^{12} \mathrm{CO}$ and ${ }^{13} \mathrm{CO}$ returned by a given RADEX model, $s^{\text {obs }}$ the observational intensity, and $\sigma$ the uncertainty associated with the measurements of the intensity $s$.

3. The physical conditions for each average spectrum were deduced from the minimization of the quantity $\chi^{2}$, that is, we localized the minimum element of the resulting $\chi_{\text {RADEX }}^{2}$ grid and inferred the quantities $\left(N, n_{\mathrm{H}_{2}}, T\right)$ from the corresponding RADEX input.

The error bars on column density and kinetic temperature were estimated from the uncertainties on the flux and more importantly from the uncertainty on the isotopic ratio ${ }^{12} \mathrm{CO} /{ }^{13} \mathrm{CO}$.

Results. The results of our LVG analysis are presented in Table 7, in which the minimum and maximum boundaries are given for the CO column density and gas mass for the shocked clump, shocked knot, ring-like structure, cloudlet, and ambient cloud. As previously, we measured the molecular mass assuming that the $\mathrm{H}_{2}-\mathrm{to}_{-}{ }^{12} \mathrm{CO}$ abundance ratio is equal to $10^{4}$. The $\chi^{2}$ minimization was successfully attempted for the major part of our analysis. We present the $\chi^{2}$ diagrams for each region in Fig. 12, where the first and second term of Eq. (12) are independently represented by two sets of filled contours. For most structures, we were not able to determine a precise measurement of the local density from the $\chi^{2}$ minimization, as the variation of $\chi^{2}$ with respect to the choice of the input $n_{\mathrm{H}_{2}}$ does not strongly favor any LVG model. In all cases we observe that $\chi^{2}$ increases significantly for densities $n_{\mathrm{H}_{2}}<10^{4} \mathrm{~cm}^{-3}$, hence our analysis suggests that (i) the ${ }^{12} \mathrm{CO}$ lines are thermalized; (ii) the local density is greater than $10^{4} \mathrm{~cm}^{-3}$ across the field of observations.

\subsection{Discussion}

The gas masses measured with the LVG approach are systematically lower than the masses obtained using population diagrams corrected for optical depth (Table 5), except for the high-velocity wings for which the LVG estimate is higher by a factor $\sim 3$ and for the cloudlet for which both methods yield the same result. Deviation from a single excitation temperature is not sufficient to account for this discrepancy, since the LVG models that fit our data do not predict a disagreement higher than $\sim 1 \mathrm{~K}$ for the excitation temperatures of ${ }^{12} \mathrm{CO}$ and ${ }^{13} \mathrm{CO}$. This discrepancy is mainly due to the fact that the LVG method is applied to average spectra, whereas the LTE approach is applied pixel per pixel and channel per channel. The channel-per-channel measures of optical depth are particularly different from the average measures toward the shocked clump for which the optical depth varies strongly between the center of the line and high-velocity wings. The highest estimates of the mass are obtained using the COto- $\mathrm{H}_{2}$ conversion factor based on the emission of ${ }^{12} \mathrm{CO} J=1-0$ only. In comparison to previous measures of the molecular mass in the extended $\mathrm{G}$ region:

- Dickman et al. (1992) measured a mass of $41.6 M_{\odot}$ for the clump $\mathrm{G}$ assuming that ${ }^{12} \mathrm{CO} J=1-0$ emission is optically thin.

- Using a CO-to- $\mathrm{H}_{2}$ conversion factor, Lee et al. (2012) measured a mass of $57.7 \pm 0.9 M_{\odot}$ for the cloudlet.

- Xu et al. (2011) measured a mass of $2.06 \times 10^{3} M_{\odot}$ for cloud $\mathrm{G}$ from the ${ }^{12} \mathrm{CO} J=3-2$ line. Their measure included a larger field, and they lacked sufficient data to correctly estimate the optical depth.

Our new measurements of the mass are crucial for the interpretation of the interaction of CRs with the ISM. The extended G region is likely to be the main target of interaction with CRs, hence the source of teraelectronvolt $\gamma$-ray emission in IC443. Our results put constraints on the amount of molecular mass that is available to interact via bremsstrahlung and pion decay mechanisms. Torres et al. (2010) showed that the characteristics of the $\gamma$-ray spectra in IC443 suggest the interaction with two distinct molecular structures: a lower-mass cloud $\left(\sim 350 M_{\odot}\right)$ at distance of $4 \mathrm{pc}$ from the SNR and a higher-mass cloud $\left(\sim 4000 M_{\odot}\right)$ at a distance of $10 \mathrm{pc}$. Their results were obtained from the analysis of the $\gamma$-ray spectra of the SNR in a larger field, yet they are consistent with our findings if we consider that the mass of the ambient gas that we measured could contribute to the distant component. As has been suggested by Lee et al. (2012), either the shocked clump or cloudlet and ring-like structure could correspond to the closer component, while the distant component might correspond to the ambient cloud. Within error bars, the mass we measured for these structures could fit with this scenario. 

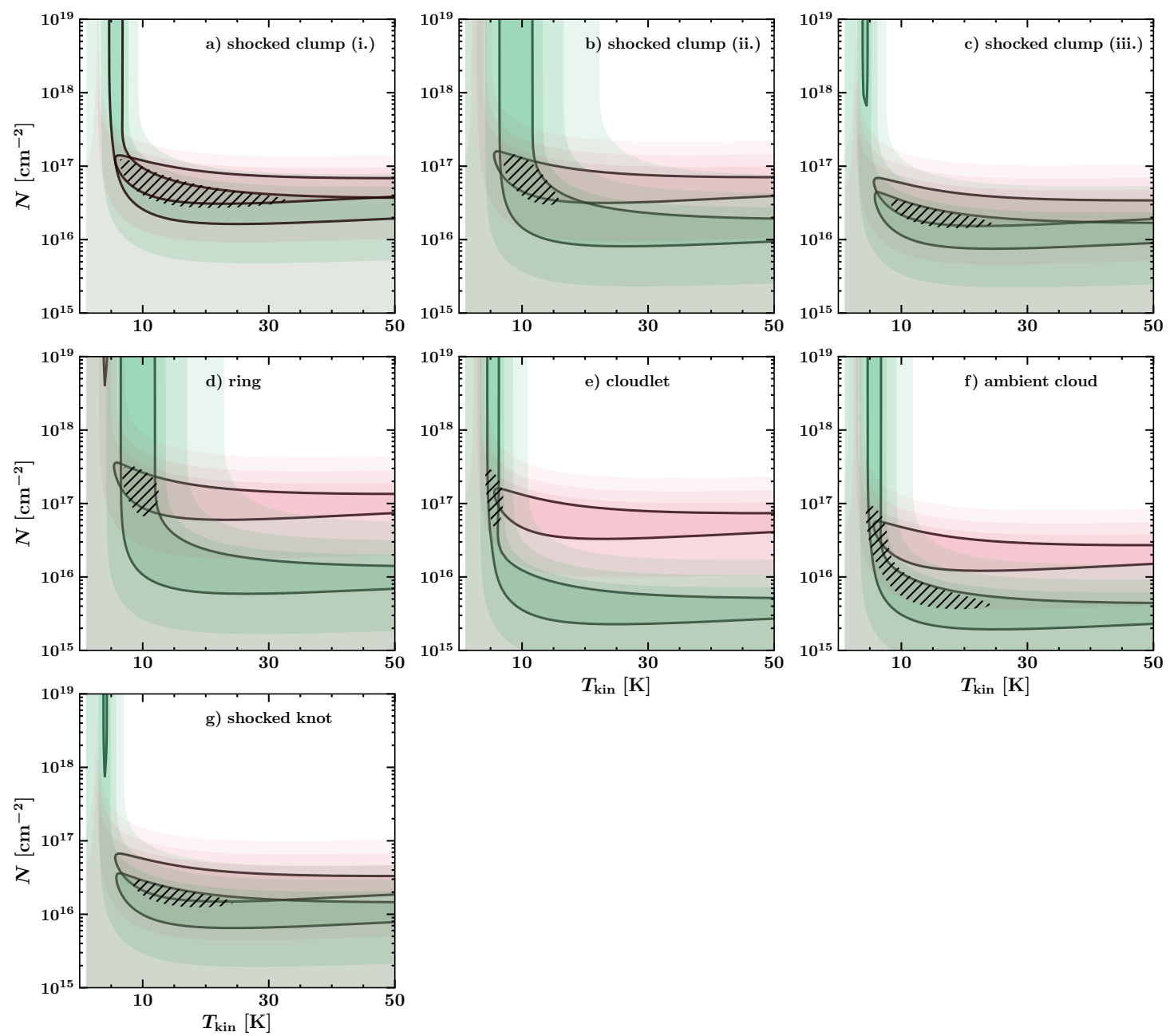

$T_{\text {kin }}[\mathrm{K}]$

$T_{\text {kin }}[\mathrm{K}]$

Fig. 12. Diagrams of $\chi^{2}$ minimization of the comparison between $1 \times 200 \times 200 \mathrm{LVG}$ grids modeled with sets of parameters $\left(n_{\mathrm{H}_{2}}, N_{\mathrm{CO}}, T\right)$ and observations of ${ }^{12} \mathrm{CO}$ and ${ }^{13} \mathrm{CO}$ lines toward the boxes corresponding to the shocked clump $(a-c)$, ring-like structure $(d)$, cloudlet $(e)$, ambient cloud $(f)$, and shocked knot $(g)$ defined in Table A.1. Shades of green represent the areas of minimum $\chi^{2}$ corresponding to the minimization of the specific intensity of the ${ }^{12} \mathrm{CO}(3-2),{ }^{12} \mathrm{CO}(2-1)$, and ${ }^{12} \mathrm{CO}(1-0)$ lines (first term introduced in Eq. (12)), and the shades of pink represent the areas of minimum $\chi^{2}$ corresponding to the minimization of ${ }^{13} \mathrm{CO}$ lines (second term introduced in Eq. (12)). The filled contour levels are $\log _{10}\left(\chi^{2}\right)=[0.6,1,1.5,2]$; the first contour level is highlighted by a solid black line. Hatched areas represent the intersection between the previously described sets of contours, defined by $\log _{10}\left(\chi_{\text {RADEX }}^{2}\right) \leq[0.5,0.45,0.45,0.5,0.75,0.75,0.5]$ (Eq. (12)) for boxes $a-g$, respectively.

\section{Protostar candidates}

In order to characterize the local star formation over the extent of the molecular structures characterized in the previous section, we studied the distribution of optical, infrared, and near-infrared point sources in the extended $G$ region. We aimed to check if these infrared point sources can be identified as protostars and if so, to constrain their evolutionary stage based on their infrared fluxes. Finally, we aimed to study their spatial distribution and their association with the molecular clumps found in the extended $\mathrm{G}$ region.

\subsection{Origin of the data}

Our field was fully observed both by 2MASS and WISE. 2MASS operates between 1.235 and $2.159 \mu \mathrm{m}$, and WISE between 3.4 and $22 \mu \mathrm{m}$. The exact photometric parameters for each band of 2MASS and WISE are given in Table 8.

To recover point sources from these two catalogs, we used the NASA/IPAC infrared science archive to obtain all entries in a 10 arcmin sized square box around the field center $\alpha_{[\mathrm{J} 2000]}=6^{\mathrm{h}} 16^{\mathrm{m}} 37.5^{\mathrm{s}}, \delta_{[\mathrm{J} 2000]}=+22^{\circ} 35^{\prime} 00^{\prime \prime}$, corresponding to the same field that was mapped with IRAM $30 \mathrm{~m}$ and APEX. A total of 487 point sources in the 2MASS All-Sky Point Source Catalog (PSC) and 515 point sources in the AllWISE Source Catalog were found using this query within the extended $G$ region. Both catalogs provide position coordinates and photometric measurements for each band and their uncertainties, and $\mathrm{S} / \mathrm{Ns}$, along with several flags specifying contamination by extended emission, quality of the point spread function (PSF) profile-fit, and other possible sources of bias.

\subsection{Selection of relevant IR point sources}

In order to reject false positives, we applied several selection criteria to our primary catalogs of point sources detected by 2MASS and WISE:

1. We required a complete detection in the WISE bands $W_{1}$, $W_{2}$, and 2MASS bands $J, H$, and $K$ (i.e., the measurement is not an upper limit).

2 . We selected only the sources characterized by a signal-tonoise ratio greater than 2 for the photometric bands $W_{1}, W_{2}, W_{3}$, $J, H$, and $K$. 
Table 8. Photometric parameters for each band of the telescopes WISE, 2MASS and Gaia.

\begin{tabular}{cccc}
\hline \hline Band & $\lambda(\mu \mathrm{m})$ & $\Delta \lambda(\mu \mathrm{m})$ & $F W H M\left({ }^{\prime \prime}\right)$ \\
\hline WISE $W_{1}$ & 3.35 & 0.66 & 6.1 \\
WISE $W_{2}$ & 4.60 & 1.04 & 6.4 \\
WISE $W_{3}$ & 11.56 & 5.51 & 6.5 \\
WISE $W_{4}$ & 22.09 & 4.10 & 12.0 \\
\hline 2MASS $J$ & 1.235 & 0.162 & 2.5 \\
2MASS $H$ & 1.662 & 0.251 & 2.5 \\
2MASS $K$ & 2.159 & 0.262 & 2.5 \\
\hline Band & $\lambda(\mathrm{nm})$ & $\Delta \lambda(\mathrm{nm})$ & $F W H M\left({ }^{\prime \prime}\right)$ \\
\hline Gaia $G$ & 673 & 440 & 0.4 \\
Gaia $G_{\mathrm{BP}}$ & 532 & 253 & 0.4 \\
Gaia $G_{\mathrm{RP}}$ & 797 & 296 & 0.4 \\
Gaia $G_{\mathrm{RVS}}$ & 860 & 28 & 0.4 \\
\hline
\end{tabular}

Notes. $\lambda$ is the band center, $\Delta \lambda$ the band width and FWHM is the full width half maximum of the PSF (corresponding to the seeing in the case of 2MASS.)

3. We rejected the WISE point sources that were flagged for confusion and/or contamination of the photometric bands by image artifacts.

4. We rejected the 2MASS point sources that were flagged for low quality photometric measurements.

After this selection, $214 / 515$ point sources remain for the AllWISE Source Catalog and 328/487 for the 2MASS PSC. A total of 99 point sources were detected both by 2 MASS and WISE. AllWISE point sources marked with a value of ext_flg flag different than " 0 " are indicated as extended sources. Either their morphology is not consistent with the PSF of any band or they are spatially associated with a known extended source of the 2MASS Extended Source Catalog (XSC). 2MASS point sources with gal_contam $=0$ are also sources that fall within the elliptical profile of a known extended source. A search in the 2MASS XSC catalog shows that 17 extended sources are found in our $10^{\prime} \times 10^{\prime}$ field. The shocked clump is particularly crowded, suggesting that the extended emission of bright knot of shocked material are detected by the survey in this area. The photometric measurements are likely to be contaminated by this extended emission, hence the identification of these sources as protostar candidates is uncertain. Nonetheless, we do not completely rule out these entries in the catalog since this extended emission might be produced by outflows.

\subsection{Color-color filtering}

In order to select only the point sources that could possibly be YSOs, we applied a color-color criteria to our near-infrared point source catalogs. To identify the nature of a protostar candidate detected by 2MASS, we compared the relative flux in the $J, K$, and $H$ photometric bands. We used the following empirical color criteria (Xu et al. 2011) based on the idea that protostars have an infrared excess in the $1.235-2.159 \mu \mathrm{m}$ range that determines their position in the $J H K$ color-color space and that is directly related to their evolutionary stage (Lada \& Adams 1992) as follows:

$(J-H)-1,7 \cdot(H-K)+0,450=0$

$(J-H)-0,493 \cdot(H-K)-0,439=0$

$(J-H)-1,7 \cdot(H-K)+1,400=0$

$(J-H)=0,2$.
Within the color-color diagram, this system of equations defines the color-color domains that denote the different types of sources (see Fig. 13). This method allows us to filter the sample of point sources and to produce a subset of different types of candidate YSOs as follows: classical T Tauri stars (CTTS), Herbig Ae/Be stars $(\mathrm{HAeBe})$, and other YSOs. Similarly, we used the following color-color criteria (Koenig \& Leisawitz 2014) to characterize the point sources from the relative flux in the three first bands $W_{1}, W_{2}$, and $W_{3}$. Fischer et al. (2016) proposed a slightly modified version of these filtering criteria that excludes an area of the color-color diagram that would be considered as Class II in the original diagram. That area is instead interpreted as shock emission, together with all the point sources that are beyond the left branches of the Class II and Class I areas. In this version of the diagram, there are two well-defined domains of color-color space:

1. The first region is defined by the following system of four equations that constrain the infrared excess observed in Class I YSOs:

$$
\begin{aligned}
& W_{2}-W_{3}>2,0 \\
& W_{1}-W_{2}>-0,42 \cdot\left(W_{2}-W_{3}\right)+2,2 \\
& W_{1}-W_{2}>0,46 \cdot\left(W_{2}-W_{3}\right)-0,9 \\
& W_{2}-W_{3}<4,5 .
\end{aligned}
$$

2. The second, adjacent region is defined by the following system of five equations that constrain the infrared excess observed in Class II YSOs:

$$
\begin{aligned}
& W_{1}-W_{2}>0,25 \\
& W_{1}-W_{2}<0,71 \cdot\left(W_{2}-W_{3}\right)-0,07 \\
& W_{1}-W_{2}>-1,5 \cdot\left(W_{2}-W_{3}\right)+2,1 \\
& W_{1}-W_{2}>0,46 \cdot\left(W_{2}-W_{3}\right)-0,9 \\
& W_{2}-W_{3}<4,5 .
\end{aligned}
$$

In the color-color diagram represented in Fig. 13, this system of nine equations allows us to distinguish two samples from the rest of the catalog and enables us to sort the YSO candidates, based on their infrared excess, into the two distinct expected evolutionary stages of Class I and Class II protostars.

In addition to the shocked emission area of the colorcolor diagram, Fischer et al. (2016) added the labels "polycyclic aromatic hydrocarbon (PAH) emission", "AGB stars", "Tr. (transition) disks", and "star-forming galaxies" to different parts of the diagram based on the expected emission of these objects in the WISE photometric bands. We defined arbitrary branches to separate the areas corresponding to each label and identified point sources falling into one of these areas.

After the color-color filtering of the catalogs, the total amount of remaining point sources is 79/328 for 2MASS and $65 / 214$ for WISE. Nine point sources are detected both by 2MASS and WISE. One point source is detected by Gaia and detected as a Class II protostar by WISE. The uncertainty due to extended emission and their identification in the color-color space is the following:

$2 M A S S$. Of the protostar candidates found in the 2MASS PSC, $12.7 \%$ are contaminated by extended emission; 23 CTTS candidates, $8 \mathrm{HAeBe}$ candidates, and 48 other YSO candidates are identified based on their JHK photometric measurements.

WISE. Of the protostar candidates found in the AllWISE cata$\log , 76.9 \%$ are contaminated by extended emission toward the crowded shocked clump; 53 class I protostar candidates and 12 class II protostar candidates are identified in our field. 

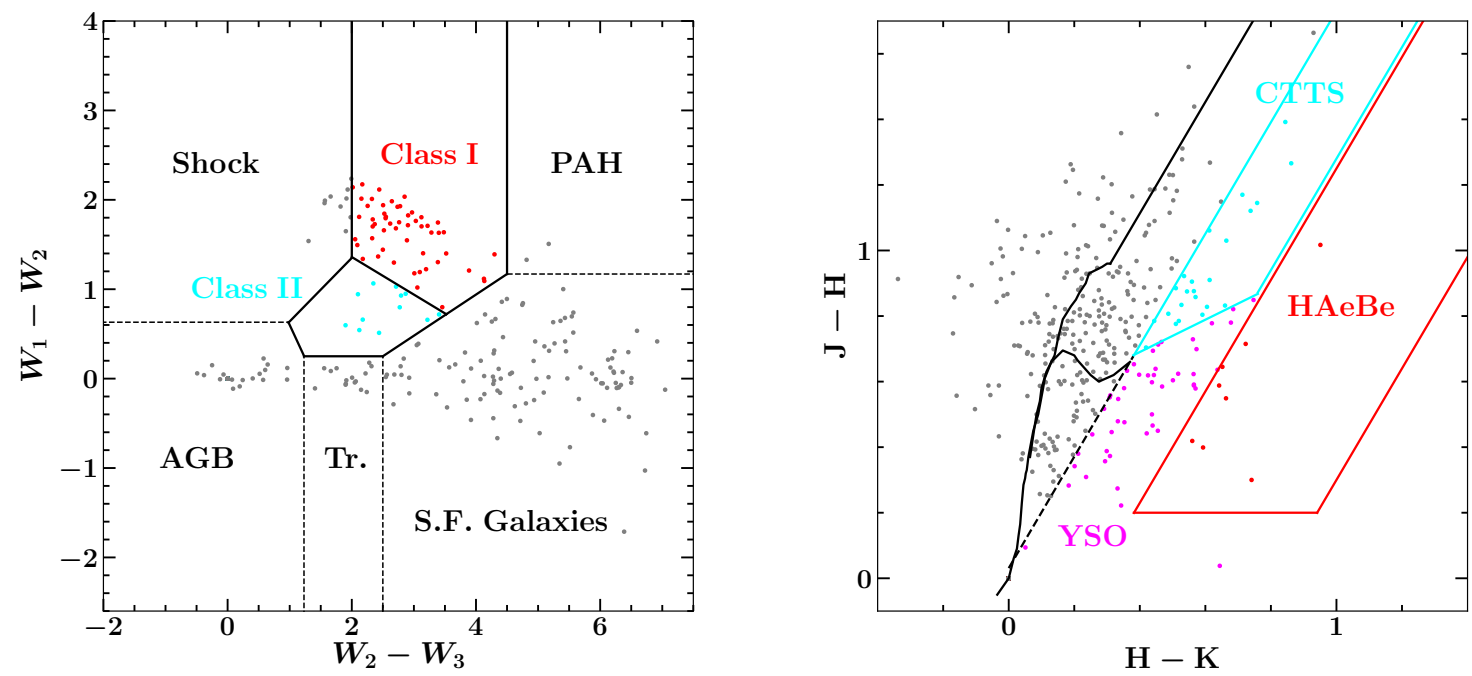

Fig. 13. Left: WISE census of protostar candidates in the extended G region. Cyan dots indicate Class II candidates, red dots indicate Class I candidates, and grey dots indicate point sources detected by WISE and rejected by our color-color filtering based on photometric bands $\mathrm{W}_{1}, \mathrm{~W}_{2}$, and $\mathrm{W}_{3}$. Right: 2MASS census of protostar candidates in the extended $\mathrm{G}$ region. Cyan dots represent CTTS candidates, red dots denote HaeBe candidates, magenta dots indicate YSO candidates, and grey dots indicate point sources detected by 2MASS and rejected by our JHK color-color filtering.

\subsection{Spectral index $\alpha$ of protostar candidates in the range 3.4-2 $\mu \mathrm{m}$}

We measured the spectral index $\alpha=\mathrm{d} \log \left(\lambda F_{\lambda}\right) / \mathrm{d} \log \lambda$ of our protostar candidates detected by $2 \mathrm{MASS}$ and WISE to derive an identification based on their infrared SED (Adams et al. 1987). We used the classification system of Greene et al. (1994) to attribute an evolutionary stages to each point source and rule out the sources characterized by a flat SED as follows:

- Class I: $\alpha \geq 0.3$

- Flat-SED: $-0.3 \leq \alpha \leq 0.3$

- Class II: $-1.6 \leq \alpha \leq-0.3$

- Class III: $\alpha<-1.6$

The photometric fluxes of the bands $\mathrm{W}_{1}, \mathrm{~W}_{2}$, and $\mathrm{W}_{3}$ were used to compute the slope of the SED in the range 3.4-12 $\mu \mathrm{m}$ for each protostar candidate found precedently and given in Table G.1, where we compare the classification based on color-color diagram with the results obtained with the measurement of $\alpha$. We show the SEDs of 17 protostar candidates in Fig. E.1. With respect to the value of the infrared spectral index $\alpha, 33$ flat-SED point sources are found within our sample of 65 AllWISE protostar candidates (ID 9-15 and ID 40-65). Every single point sources classified as Class I by our measurement of $\alpha$ were also identified as Class I using the color-color diagram (ID 1-4 and ID 16-34). On the contrary, there is only partial agreement between the two methods for the identification of Class II protostar candidates: excluding Flat-SED sources, $75 \%$ of the identifications were confirmed by both approaches (ID 5-8 and ID 35-39). No Class III were identified in our sample.

\subsection{Gaia point sources}

We used Gaia data (see Table 8 for exact photometric parameters) to complete our point source census with optical sources and search for multiple detection by Gaia, WISE, and 2MASS. We used the ESA Gaia science archive to obtain all entries in a 10 arcmin sized square box around the field center. A total of 468 point sources were found within our $10^{\prime} \times 10^{\prime}$ field of observations. In order to estimate the amount of optical point sources that might be evolved stars physically associated with the SNR, we also used a distance criterion to filter the result. Assuming that the distance of IC443 is $\sim 1.9 \mathrm{kpc}$, we rejected the point sources with relative distance greater than $2500 \mathrm{pc}$ or lower than $1500 \mathrm{pc}$, based on the lower and upper bound on the confidence interval for the estimated distance determined by parallax measurements Bailer-Jones et al. (2018). There are 16 point sources in the interval $1500-2500 \mathrm{pc}$. We repeated the same process with a different distance criterion to assess the variability of the result with respect to the amplitude of distance interval applied as a filter. We found a total of 40 optical point sources whose relative distance is greater than $1250 \mathrm{pc}$ and lower than $2750 \mathrm{pc}$. We checked if our samples of optical point sources are correlated with candidate protostars found in our WISE and 2MASS census. Among the 16 Gaia point sources found with the first distance check ( $1500 \leq d \leq 2500), 3$ point sources were also detected by 2 MASS, and 1 was spatially correlated with a WISE point source. For the 40 Gaia point sources detected with the second distance check $(1250 \leq d \leq 2750), 5$ point sources were also detected by 2 MASS, and 5 with a WISE point source. Hence a fraction of the YSO detections by WISE and 2MASS are confirmed by Gaia, and the distance estimates allow us to strengthen the assumption that these sources are physically associated with the extended $\mathrm{G}$ region.

\subsection{Discussion}

A higher concentration of protostar candidates was found within the shocked clump by our WISE census, suggesting that this might be a star-forming region. However, the third photometric band of WISE $\mathrm{W}_{3}(\lambda=11.56 \mu \mathrm{m}, \Delta \lambda=5.51 \mu \mathrm{m})$ is partially sensitive to three pure rotational transitions of $\mathrm{H}_{2}$, namely the transitions $\mathrm{S}(2)(\lambda=12.28 \mu \mathrm{m}), \mathrm{S}(3)(\lambda=9.665 \mu \mathrm{m})$, and $\mathrm{S}(4)$ $(\lambda=8.026 \mu \mathrm{m})$. Moreover, several $\mathrm{H}_{2}$ rovibrational transitions are detected by WISE bands. Hence our detections might be contaminated by the emission of warm $\mathrm{H}_{2}$ clumps that are excited by the propagation of the shock. Gutermuth et al. (2009) reported that when trying to build YSO samples, unresolved knots of shock emission are regularly detected in all Infrared Array Camera (IRAC) bands (3.6-8 $\mu \mathrm{m})$. As a consequence, warm and shocked condensations of $\mathrm{H}_{2}$ smaller than the spatial resolution 
might be detected as infrared point sources by the survey. Fischer et al. (2016) provides a quantitative distinction between shock emission and the signature of protostar candidates in the WISE color-color diagram, yet none of the point sources located along the bright shocked clump are identified as shock emission, casting doubts about the reliability of this criterion to effectively detect shocks. Also, several point sources associated with faint, extended Spitzer-MIPS $24 \mu \mathrm{m}$ northeast of the brightest shocked clump are identified as shock emission. The clumpy and filamentary morphology of the structure in which these point sources are embedded, seen in Spitzer-IRAC $4.5 \mu \mathrm{m}$, is similar to that of the main shocked clump. This spatial disparity in the identification of point sources might indicate that this region is dominated by shock emission, whereas the point sources detected in the shocked clump are dominated by protostar emission despite the contamination by extended shock structures. The 2MASS census is also biased by the emission of warm $\mathrm{H}_{2}$, as the band $\mathrm{K}$ $(\lambda=2.159 \mu \mathrm{m}, \Delta \lambda=0.262 \mu \mathrm{m})$ is sensitive to the rovibrational line $v=1-0 \mathrm{~S}(1)$ at $2.12 \mu \mathrm{m}$. The clumpy and bright extended emission displayed in the band $\mathrm{K}$ map of the southern ridge makes it clear that the flux of point sources might be contaminated by extended emission, in particular along the bright shocked clump. Rho et al. (2001) confirmed that the band $K$ extended emission from this region of the remnant is dominated by $\mathrm{H}_{2}$ emission and to a lesser extent the bands $J$ and $H$. On the one hand, if the flux of the band $\mathrm{K}$ is overestimated because of $\mathrm{H}_{2}$ emission, then the correction would lead to a displacement along the $H$ - $K$ axis, which means that more protostar candidates would be detected toward the shocked clump where the emission of warm $\mathrm{H}_{2}$ is significant. This might explain why the higher density of protostar candidates found in the WISE census is not reproduced by our 2MASS census. On the other hand, it is possible that a number of point sources detected by 2MASS are unresolved knots of shocked $\mathrm{H}_{2}$, in a similar fashion as that is expected for WISE.

A greater amount of point sources is found on the edges of the $24 \mu \mathrm{m}$ bright knot that is spatially associated with the eastern edge of the quiescent molecular cloudlet. Excluding the shocked clump, there is an anti-correlation between both 2MASS and WISE sources and the MIPS-24 $\mu$ m flux map, in particular in the vicinity of the cloudlet. We suppose that the absence of optical point sources within this region is due to the extinction caused by this massive dark cloud. No IR point sources are found at the center of the gap within the ring-like structure.

Interestingly, the few point sources that were not flagged for contamination by extended sources are primarily organized in two clusters in the eastern and northeastern part of the field (group 1 and group 2, Fig. 14). All these candidates were positively identified by both methods as Class I and Class II protostars. The Class I protostar candidates of group 2 are well associated with Spitzer-IRAC filamentary structures at $4.5 \mu \mathrm{m}$, at the edge of a faint structure detected by Spitzer-MIPS at $24 \mu \mathrm{m}$. This bright filament can be either part of the SNR shock or unresolved outflows. Most IR sources with protostar signatures are spatially correlated with the molecular shell in IC443G, suggesting enhanced star formation along the rims. Given the protostellar collapse phase timescale of $\sim 10^{5} \mathrm{yr}$ (Lefloch \& Lazareff 1994) we do not establish any causal relation between the SNR shocks and the formation of Class I and Class II protostars. Based on our color-color census for the 65 protostar candidates detected by WISE, the Class II/Class I ratio is equal to 0.23. Following Dunham et al. (2015), we assumed a Class II duration of $2 \mathrm{Myr}$ and used the standard method to infer the age of a stellar population from this ratio (Wilking et al.
1989; Evans et al. 2009). We obtain an age of $500 \mathrm{kyr}$ for the YSO population found in the extended $\mathrm{G}$ region, which is not consistent with the scenario in which the formation of these YSOs was triggered by interaction of the SNR with its environment. It is likely that the collapse of molecular clouds in the vicinity of the high-mass progenitor was triggered by the compression driven by stellar winds (Xu et al. 2011). Finding evidence of enhanced star formation in the SNR shocks would require us to detect deeply embedded prestellar cores, which is not attainable with the data at our disposal. In the future, highresolution $\left(\sim 1^{\prime \prime}\right)$ submillimeter and millimeter observations will be required to disentangle the kinematics of large-scale shock structure and outflows, and to eventually uncover prestellar cores in the extended $\mathrm{G}$ region (e.g., as Motte et al. 2018 did for the W43-MM1 star-forming region). Still, our sample of protostar candidates provides a unique opportunity to study star formation in an environment that is subject to intense $\gamma$-ray flux and shock dynamical feedback. The protons accelerated by young protostars might also provide a source of fresh mid-energy CRs (up to $10-13$ and $26-37 \mathrm{GeV}$ for jet acceleration and protostellar surface shock acceleration in lower-mass stars, respectively Padovani et al. 2016). With jet velocities up to $1000 \mathrm{~km} \mathrm{~s}^{-1}$, highmass protostellar shocks could contribute to the teraelectronvolt $\gamma$-ray peak in the extended $\mathrm{G}$ region.

Point sources identified as star-forming galaxies from their location in the color-color diagram display a significant correlation with the spiral-shaped, bright, and extended infrared features mapped by Spitzer-MIPS at $24 \mu \mathrm{m}$. Most of these sources might as well be identified as PAH emission, as our arbitrary criterion of selection puts a significant uncertainty on the cut between the two categories of sources, in particular along the $\mathrm{W}_{1}-\mathrm{W}_{2}$ axis (Koenig et al. 2012). In fact, it is more likely that these point sources are associated with PAH, stellar, or protostellar emission. With our current criterion, only two sources identified as PAH emission are found and located in the same area as point sources that were labeled star-forming galaxies. If most of these detections are actually arising from PAH emission, this result would support the assumption that the bright infrared extended emission arise from a dark dust lane that is spatially associated with the molecular cloudlet detected by our CO observations.

The findings of two relevant studies can be compared to the results presented in this section. Based on 2MASS point source catalogs, Xu et al. (2011) found a total of 1666 YSO candidates, 154 CTTS, and 419 HAeBe stars in a search circle around IC443 within a $25^{\prime}$ radius. Their candidates were mostly concentrated around the $\mathrm{CO}$ molecular shell, in particular, toward clump $\mathrm{C}$ and the extended $\mathrm{G}$ region where their YSO candidates are spatially correlated with the shocked clump. These authors proposed that the formation of these YSOs were triggered by the stellar wind of the IC443 progenitor. Based on 2MASS and WISE point source catalogs, Su et al. (2014) found a total of 98 YSO candidates. They proposed a sample of 62 YSO candidates concentrated along the boundary of the radio shell. They also proposed that the formation of these protostars is likely to have been triggered by the stellar winds of the SNR progenitor. In contrast with our results and the findings of $\mathrm{Xu}$ et al. (2011), their distribution does not show a strong correlation with the molecular structures in the extended G region.

\section{Summary}

In this work we report on new $10^{\prime} \times 10^{\prime}$ fully sampled maps of the extended IC443G region for the first three rotational 
P. Dell'Ova et al.: Interstellar anatomy of the TeV gamma-ray peak in the IC443 supernova remnant
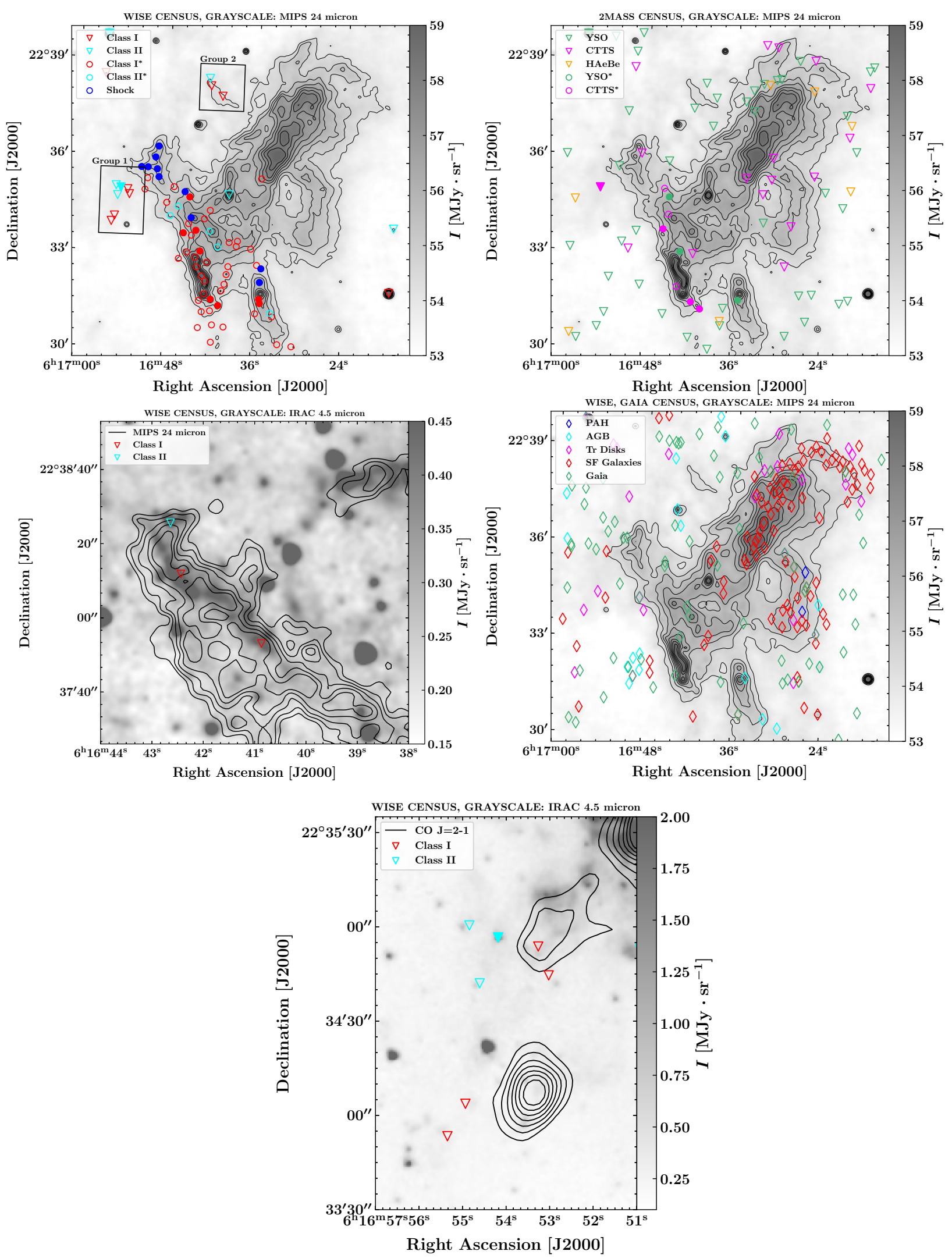

Fig. 14. Top left panel: WISE census of Class I and Class II protostar candidates, with shock emission indicated. Based on the selection criteria by Fischer et al. (2016). Top right panel: 2MASS census of YSO, CTTS, and HAeBe candidates. Based on the selection criteria by Xu et al. (2011). Center left panel: close-up on the box corresponding to group 2. Center right panel: transition disks, AGB star, star-forming galaxies emission, and PAH emission candidates, based on the selection criteria by Fischer et al. (2016); and Gaia point sources. Bottom panel: close-up on the box corresponding to group 1. On all panels, triangle-shaped markers correspond to well-detected point sources, and circle-shaped markers (labels marked with a “*”) correspond to point sources that were flagged for contamination by extended emission. Filled markers represent point sources that were detected both by 2 MASS and WISE. 
transitions of both ${ }^{12} \mathrm{CO}$ and ${ }^{13} \mathrm{CO}$, as well as the two first rotational transitions of $\mathrm{C}^{18} \mathrm{O}$ obtained with the IRAM $30 \mathrm{~m}$ and APEX telescope. These maps allow us to probe the position of the teraelectronvolt $\gamma$-ray peak in IC443G and its surroundings with unprecedented spectral and angular resolution at millimeter and submillimeter wavelengths. We proposed a description of the morphology and kinematics of the extended $G$ region based on the definition of four main molecular structures: (i) a shocked clump, (ii) a quiescent, ring-like structure that might be spurious, (iii) a quiescent cloudlet that is spatially connected to the ring-like structure, and (iv) a shocked knot. Based on this morphological description, our results are the following:

1. The comparison of our data histograms with modified LTE radiative transfer models revealed enhanced ${ }^{12} \mathrm{CO} J=2-$ $1 / 1-0$ and $J=3-2 / 2-1$ ratios toward the shocked clump, in particular within the high-velocity wings of the emission lines that are tracing warm CO $(40-120 \mathrm{~K})$. We provide a rough estimate of the isotopic ratio based on the best match between modified LTE models and the measured intensity of the ${ }^{12} \mathrm{CO},{ }^{13} \mathrm{CO}$, and $\mathrm{C}^{18} \mathrm{O} J=1-0$ line.

2. We measured the mass of the molecular gas in the extended $\mathrm{G}$ region using pixel-per-pixel, channel-per-channel population diagrams corrected for optical depth. We obtained a total molecular mass estimate of $\sim 230 M_{\odot}, \sim 90 M_{\odot}, \sim 210 M_{\odot}$ and $\sim 4 M_{\odot}$ for the cloudlet, ring-like structure, shocked clump, and shocked knot, respectively. We also measured a mass of $\sim 1100 M_{\odot}$ for the ambient gas in the $[-6.5,-1.5] \mathrm{km} \mathrm{s}^{-1}$ $v_{\mathrm{LSR}}$ range. The estimate of the mass depends on the adopted ${ }^{12} \mathrm{CO} /{ }^{13} \mathrm{CO}$ isotopic ratio, but it is established that a total molecular mass $0.9-3.1 \times 10^{3} M_{\odot}$ is available to interact with $\mathrm{CRs}$ via pion-decay in the extended $\mathrm{G}$ region.

3. We proposed a second estimate of the mass using the LVG assumption with a grid of RADEX models. The $\chi^{2}$ minimization of the grid of models with respect to data in each structure yields results that are in partial agreement with the previous measurements, with an estimate that is systematically lower than the LTE estimate, except for the mass measurements of the high-velocity wings toward the shocked clump, which are higher by a factor $\sim 3$.

4. We studied the SED of infrared and optical point sources using the 2MASS, WISE, and Gaia catalogs of point sources. Using color-color diagrams, we determined a sample of protostar candidates in the extended $\mathrm{G}$ region. We found 144 protostar candidates in that region and 16 stars in the Gaia census but a large fraction of these point sources are contaminated by extended emission (12.7\% for 2MASS, and $76.9 \%$ for WISE). Based on the spatial distribution of these candidates, we propose three sites of possible star formation in IC443G (groups 1 and 2 in Fig. 14, and the shocked clump).

Our results indicate that the shocked clump holds only a fraction of the total molecular mass in the extended $G$ region, hence the ring-like structure and the cloudlet should be taken into account to quantify the interaction of CRs with the dense local medium. Furthermore, the candidate protostars found in the region might constitute a source of CR injection that could contribute to the peak of $\gamma$-ray emission in IC443G.

Acknowledgments. This work was supported by the Programme National "Physique et Chimie du Milieu Interstellaire" (PCMI) of CNRS/INSU with INC/INP co-funded by CEA and CNES. This publication is based on observations made with ESO Telescopes at the Atacama Pathfinder EXperiment under programme M-0102.F-9508A-2018. This work has made use of data from the European Space Agency (ESA) mission Gaia (https:// www.cosmos.esa.int/gaia), processed by the Gaia Data Processing and
Analysis Consortium (DPAC, https://www.cosmos.esa.int/web/gaia/ dpac/consortium). Funding for the DPAC has been provided by national institutions, in particular the institutions participating in the Gaia Multilateral Agreement. This research has made use of the NASA/ IPAC Infrared Science Archive, which is operated by the Jet Propulsion Laboratory, California Institute of Technology, under contract with the National Aeronautics and Space Administration. This publication makes use of data products from the Wide-field Infrared Survey Explorer, which is a joint project of the University of California, Los Angeles, and the Jet Propulsion Laboratory/California Institute of Technology, funded by the National Aeronautics and Space Administration. This publication makes use of data products from the Two Micron All Sky Survey, which is a joint project of the University of Massachusetts and the Infrared Processing and Analysis Center/California Institute of Technology, funded by the National Aeronautics and Space Administration and the National Science Foundation. This research made use of Astropy (http: //www . astropy . org), a communitydeveloped core Python package for Astronomy (Astropy Collaboration 2013, 2018). This research has made use of NASA's Astrophysics Data System. M.P. acknowledges funding from the INAF PRIN-SKA 2017 program 1.05.01.88.04.

\section{References}

Abdo, A. A., Ackermann, M., Ajello, M., et al. 2010, ApJ, 712, 459 Ackermann, M., Ajello, M., Allafort, A., et al. 2013, Science, 339, 807 Adams, F. C., Lada, C. J., \& Shu, F. H. 1987, ApJ, 312, 788

Alarie, A., \& Drissen, L. 2019, MNRAS, 489, 3042

Albert, J., Aliu, E., Anderhub, H., et al. 2007, ApJ, 664, L87

Ambrocio-Cruz, P., Rosado, M., de la Fuente, E., Silva, R., \& Blanco-Piñon, A. 2017, MNRAS, 472, 51

Astropy Collaboration (Robitaille, T. P., et al.) 2013, A\&A, 558, A33

Astropy Collaboration (Price-Whelan, A. M., et al.) 2018, AJ, 156, 123

Bailer-Jones, C. A. L., Rybizki, J., Fouesneau, M., Mantelet, G., \& Andrae, R. 2018, AJ, 156, 58

Bocchino, F., \& Bykov, A. M. 2000, A\&A, 362, L29

Bocchino, F., \& Bykov, A. M. 2003, A\&A, 400, 203

Bolatto, A. D., Wolfire, M., \& Leroy, A. K. 2013, ARA\&A, 51, 207

Braun, R., \& Strom, R. G. 1986, A\&A, 164, 193

Bron, E., Daudon, C., Pety, J., et al. 2018, A\&A, 610, A12

Burton, M. G., Hollenbach, D. J., Haas, M. R., \& Erickson, E. F. 1990, ApJ, 355, 197

Bykov, A. M., Ellison, D. C., Marcowith, A., \& Osipov, S. M. 2018, Space Sci. Rev., 214, 41

Cardillo, M., Amato, E., \& Blasi, P. 2016, A\&A, 595, A58

Carter, M., Lazareff, B., Maier, D., et al. 2012, A\&A, 538, A89

Castelletti, G., Dubner, G., Clarke, T., \& Kassim, N. E. 2011, A\&A, 534, A21

Celli, S., Morlino, G., Gabici, S., \& Aharonian, F. A. 2019, MNRAS, 487, 3199

Cesarsky, D., Cox, P., Pineau des Forêts, G., et al. 1999, A\&A, 348, 945

Cherchneff, I. 2014, ArXiv e-prints, [arXiv:1405.1216]

Chevalier, R. A. 1974, ApJ, 188, 501

Claussen, M. J., Frail, D. A., Goss, W. M., \& Gaume, R. A. 1997, ApJ, 489, 143 Dame, T. M., Hartmann, D., \& Thaddeus, P. 2001, ApJ, 547, 792

Denoyer, L. K. 1978, MNRAS, 183, 187

Denoyer, L. K. 1979a, ApJ, 232, L165

Dickman, R. L., Snell, R. L., Ziurys, L. M., \& Huang, Y.-L. 1992, ApJ, 400, 203

Draine, B. T. 2011, Physics of the Interstellar and Intergalactic Medium (Princeton: Princeton University Press)

Dunham, M. M., Allen, L. E., Evans, Neal J., I., et al. 2015, ApJS, 220, 11

Egron, E., Pellizzoni, A., Iacolina, M. N., et al. 2017, MNRAS, 470, 1329

Endres, C. P., Schlemmer, S., Schilke, P., Stutzki, J., \& Müller, H. S. P. 2016, J.

Mol. Spectr., 327, 95

Esposito, J. A., Hunter, S. D., Kanbach, G., \& Sreekumar, P. 1996, ApJ, 461, 820 Evans, Neal J., I., Dunham, M. M., Jørgensen, J. K., et al. 2009, ApJS, 181, 321 Fesen, R. A., \& Kirshner, R. P. 1980, ApJ, 242, 1023

Fischer, W. J., Padgett, D. L., Stapelfeldt, K. L., \& Sewiło, M. 2016, ApJ, 827, 96

Frail, D. A., Goss, W. M., Reynoso, E. M., et al. 1996, AJ, 111, 1651

François, P., Matteucci, F., Cayrel, R., et al. 2004, A\&A, 421, 613

Frerking, M. A., Langer, W. D., \& Wilson, R. W. 1982, ApJ, 262, 590

Gabici, S., Aharonian, F. A., \& Casanova, S. 2009, MNRAS, 396, 1629

Gabici, S., Evoli, C., Gaggero, D., et al. 2019, Int. J. Mod. Phys. D., 28, 1930022-339

Glassgold, A. E., Huggins, P. J., \& Langer, W. D. 1985, ApJ, 290, 615

Goldsmith, P. F., \& Langer, W. D. 1999, ApJ, 517, 209

Gordon, M. A., \& Burton, W. B. 1976, ApJ, 208, 346

Goss, W. M. 1968, ApJS, 15, 131

Greene, T. P., Wilking, B. A., Andre, P., Young, E. T., \& Lada, C. J. 1994, ApJ, 434, 614

Grenier, I. A., Black, J. H., \& Strong, A. W. 2015, ARA\&A, 53, 199 
Gutermuth, R. A., Megeath, S. T., Myers, P. C., et al. 2009, ApJS, 184, 18

Hanabata, Y., Katagiri, H., Hewitt, J. W., et al. 2014, ApJ, 786, 145

Harris, D. E. 1962, ApJ, 135, 661

Herbst, W., \& Assousa, G. E. 1977, ApJ, 217, 473

Hester, J. J. 1987, ApJ, 314, 187

Hewitt, J. W., Yusef-Zadeh, F., Wardle, M., Roberts, D. A., \& Kassim, N. E. 2006, ApJ, 652, 1288

Hewitt, J. W., Yusef-Zadeh, F., \& Wardle, M. 2008, ApJ, 683, 189

Hewitt, J. W., Yusef-Zadeh, F., \& Wardle, M. 2009, ApJ, 706, L270

Heyminck, S., Kasemann, C., Güsten, R., de Lange, G., \& Graf, U. U. 2006, A\&A, 454, L21

Hezareh, T., Wiesemeyer, H., Houde, M., Gusdorf, A., \& Siringo, G. 2013, A\&A, 558, A45

Hildebrand, R. H. 1983, QJRAS, 24, 267

Hoffman, I. M., Goss, W. M., Brogan, C. L., Claussen, M. J., \& Richards, A. M. S. 2003, ApJ, 583, 272

Hollenbach, D., \& McKee, C. F. 1989, ApJ, 342, 306

Huang, Y. L., Dickman, R. L., \& Snell, R. L. 1986, ApJ, 302, L63

Hunter, J. D. 2007, Comput. Sci. Eng., 9, 90

Indriolo, N., Blake, G. A., Goto, M., et al. 2010, ApJ, 724, 1357

Jones, E., Oliphant, T., Peterson, P., et al. 2001, SciPy: Open Source Scientific Tools for Python

Klein, B., Hochgürtel, S., Krämer, I., et al. 2012, A\&A, 542, L3

Koenig, X. P., \& Leisawitz, D. T. 2014, ApJ, 791, 131

Koenig, X. P., Leisawitz, D. T., Benford, D. J., et al. 2012, ApJ, 744, 130

Koo, B.-C., McKee, C. F., Lee, J.-J., et al. 2008, ApJ, 673, L147

Koo, B.-C., Heiles, C., Stanimirović, S., \& Troland, T. 2010, AJ, 140, 262

Lada, C. J., \& Adams, F. C. 1992, ApJ, 393, 278

Langer, W. D., \& Penzias, A. A. 1993, ApJ, 408, 539

Larson, R. B. 1981, MNRAS, 194, 809

Lee, J.-J., Koo, B.-C., Yun, M. S., et al. 2008, AJ, 135, 796

Lee, J.-J., Koo, B.-C., Snell, R. L., et al. 2012, ApJ, 749, 34

Lefloch, B., \& Lazareff, B. 1994, A\&A, 289, 559

Lockett, P., Gauthier, E., \& Elitzur, M. 1999, ApJ, 511, 235

Loru, S., Pellizzoni, A., Egron, E., et al. 2019, MNRAS, 482, 3857

Mac Low, M.-M., \& Klessen, R. S. 2004, Rev. Mod. Phys., 76, 125

Madau, P., \& Dickinson, M. 2014, ARA\&A, 52, 415

Micelotta, E. R., Matsuura, M., \& Sarangi, A. 2018, Space Sci. Rev., 214, 53

Mihalas, D. 1978, Stellar Atmospheres (San Francisco, USA: W.H. Freeman)

Minkowski, R. 1946, PASP, 58, 305

Montmerle, T. 1979, ApJ, 231, 95

Moorhouse, A., Brand, P. W. J. L., Geballe, T. R., \& Burton, M. G. 1991, MNRAS, 253, 662

Motte, F., Nony, T., Louvet, F., et al. 2018, Nat. Astron., 2, 478

Muders, D., Hafok, H., Wyrowski, F., et al. 2006, A\&A, 454, L25

Mufson, S. L., McCollough, M. L., Dickel, J. R., et al. 1986, AJ, 92, 1349

Müller, H. S. P., Thorwirth, S., Roth, D. A., \& Winnewisser, G. 2001, A\&A, 370, L49

Müller, H. S. P., Schlöder, F., Stutzki, J., \& Winnewisser, G. 2005, J. Mol. Struct. 742,215

Neufeld, D., Bergin, E., Hollenbach, D., et al. 2004, IRS Spectroscopy of Shocked Molecular Gas in Supernova Remnants: Probing the Interaction of a Supernova with a Molecular Cloud, Spitzer Proposal

Neufeld, D. A., Hollenbach, D. J., Kaufman, M. J., et al. 2007, ApJ, 664, 890

Noriega-Crespo, A., Hines, D. C., Gordon, K., et al. 2009, in The Evolving ISM in the Milky Way and Nearby Galaxies, 46

Olbert, C. M., Clearfield, C. R., Williams, N. E., Keohane, J. W., \& Frail, D. A 2001a, ApJ, 554, L205

Olbert, C. M., Clearfield, C. R., Williams, N. E., Keohane, J. W., \& Frail, D. A 2001b, AIP Conf. Ser., 565, 341
Padovani, M., Hennebelle, P., Marcowith, A., \& Ferrière, K. 2015, A\&A, 582, L13

Padovani, M., Marcowith, A., Hennebelle, P., \& Ferrière, K. 2016, A\&A, 590, A8

Padovani, M., Marcowith, A., Sánchez-Monge, Á., Meng, F., \& Schilke, P. 2019 A\&A, 630, A72

Parker, R. A. R. 1963, PhD thesis, California Institute of Technology, USA

Parker, R. A. R. 1964, ApJ, 139, 493

Petre, R., Szymkowiak, A. E., Seward, F. D., \& Willingale, R. 1988, ApJ, 335, 215

Pickett, H. M., Poynter, R. L., Cohen, E. A., et al. 1998, J. Quant. Spectr. Rad. Transf., 60, 883

Pinheiro Gonçalves, D., Noriega-Crespo, A., Paladini, R., Martin, P. G., \& Carey, S. J. 2011, AJ, 142, 47

Reach, W. T., Tram, L. N., Richter, M., Gusdorf, A., \& DeWitt, C. 2019, ApJ, 884,81

Rho, J., \& Petre, R. 1998, ApJ, 503, L167

Rho, J., Jarrett, T. H., Cutri, R. M., \& Reach, W. T. 2001, ApJ, 547, 885

Roueff, A., Gerin, M., Gratier, P., et al. 2020, A\&A, in press, https:// doi.org/ 10.1051/0004-6361/202037776

Sarangi, A., Matsuura, M., \& Micelotta, E. R. 2018, Space Sci. Rev., 214, 63

Seta, M., Hasegawa, T., Dame, T. M., et al. 1998, ApJ, 505, 286

Shinn, J.-H., Koo, B.-C., Seon, K.-I., \& Lee, H.-G. 2011, ApJ, 732, 124

Snell, R. L., Hollenbach, D., Howe, J. E., et al. 2005, ApJ, 620, 758

Sobolev, V. V. 1960, Moving Envelopes of Stars (Harvard University Press)

Su, Y., Fang, M., Yang, J., Zhou, P., \& Chen, Y. 2014, ApJ, 788, 122

Surdej, J. 1977, A\&A, 60, 303

Tang, X. 2019, MNRAS, 482, 3843

Tatischeff, V., \& Gabici, S. 2018, Ann. Rev. Nucl. Part. Sci., 68, 377

Tauber, J. A., Snell, R. L., Dickman, R. L., \& Ziurys, L. M. 1994, ApJ, 421, 570

Tavani, M., Giuliani, A., Chen, A. W., et al. 2010, ApJ, 710, L151

Torres, D. F., Marrero, A. Y. R., \& de Cea Del Pozo, E. 2010, MNRAS, 408, 1257

Troja, E., Bocchino, F., \& Reale, F. 2006, ApJ, 649, 258

Troja, E., Bocchino, F., Miceli, M., \& Reale, F. 2008, A\&A, 485, 777

van der Tak, F. F. S., Black, J. H., Schöier, F. L., Jansen, D. J., \& van Dishoeck, E. F. 2007, A\&A, 468, 627

van der Walt, S., Colbert, S. C., \& Varoquaux, G. 2011, Comput. Sci. Eng., 13, 22

van Dishoeck, E. F., Jansen, D. J., \& Phillips, T. G. 1993, A\&A, 279, 541

VERITAS Collaboration (Humensky, B.) 2015, 34th International Cosmic Ray Conference (ICRC2015), 875

Wang, Z., \& Scoville, N. Z. 1992, ApJ, 386, 158

Wang, Z. R., Asaoka, I., Hayakawa, S., \& Koyama, K. 1992, PASJ, 44, 303

Wardle, M. 1999, ApJ, 525, L101

Wardle, M., \& Yusef-Zadeh, F. 2002, Science, 296, 2350

Welsh, B. Y., \& Sallmen, S. 2003, A\&A, 408, 545

Wenger, T. V., Balser, D. S., Anderson, L. D., \& Bania, T. M. 2018, ApJ, 856, 52

White, G. J., Rainey, R., Hayashi, S. S., \& Kaifu, N. 1987, A\&A, 173, 337

Wilking, B. A., Lada, C. J., \& Young, E. T. 1989, ApJ, 340, 823

Wilson, T. L., \& Rood, R. 1994, ARA\&A, 32, 191

Wood, C. A., Mufson, S. L., \& Dickel, J. R. 1991, AJ, 102, 224

Wright, E. L., Eisenhardt, P. R. M., Mainzer, A. K., et al. 2010, AJ, 140, 1868

Xu, J.-L., Wang, J.-J., \& Miller, M. 2011, ApJ, 727, 81

York, D. G., Adelman, J., Anderson, John E., J., et al. 2000, AJ, 120, 1579

Yusef-Zadeh, F., Wardle, M., Rho, J., \& Sakano, M. 2003, ApJ, 585, 319

Zhang, Z., Gao, Y., \& Wang, J. 2010, Sci. China Phys. Mech. Astron., 53, 1357

Ziurys, L. M., Snell, R. L., \& Dickman, R. L. 1988, Recent Molecular Studies of Supernova Remnant IC443 - Some New Results for Shock Chemistry, eds. R. L. Dickman, R. L. Snell, \& J. S. Young, 315, 184 


\section{Appendix A: Coordinates of the boxes}

Table A.1. Coordinates of the vertices defining the boxes corresponding to dense cloudlet, ring-like structure, and shocked clump (Fig. 6, Table A.1).

\begin{tabular}{ccc}
\hline \hline Region & RA $[\mathrm{J} 2000]$ & Dec [J2000] \\
\hline & $6^{\mathrm{h}} 16^{\mathrm{m}} 37.0^{\mathrm{s}}$ & $+22^{\circ} 40^{\prime} 24.0^{\prime \prime}$ \\
& $6^{\mathrm{h}} 16^{\mathrm{m}} 23.1^{\mathrm{s}}$ & $+22^{\circ} 40^{\prime} 24.0^{\prime \prime}$ \\
Dense cloudlet & $6^{\mathrm{h}} 16^{\mathrm{m}} 23.1^{\mathrm{s}}$ & $+22^{\circ} 33^{\prime} 39.8^{\prime \prime}$ \\
$(\mathrm{A})$ & $6^{\mathrm{h}} 16^{\mathrm{m}} 30.3^{\mathrm{s}}$ & $+22^{\circ} 33^{\prime} 39.8^{\prime \prime}$ \\
& $6^{\mathrm{h}} 16^{\mathrm{m}} 30.3^{\mathrm{s}}$ & $+22^{\circ} 36^{\prime} 09.5^{\prime \prime}$ \\
& $6^{\mathrm{h}} 16^{\mathrm{m}} 37.0^{\mathrm{s}}$ & $+22^{\circ} 36^{\prime} 09.5^{\prime \prime}$ \\
\hline & $6^{\mathrm{h}} 16^{\mathrm{m}} 41.2^{\mathrm{s}}$ & $+22^{\circ} 36^{\prime} 09.5^{\prime \prime}$ \\
Ring-like structure & $6^{\mathrm{h}} 16^{\mathrm{m}} 30.3^{\mathrm{s}}$ & $+22^{\circ} 36^{\prime} 09.5^{\prime \prime}$ \\
$(\mathrm{B})$ & $6^{\mathrm{h}} 16^{\mathrm{m}} 30.3^{\mathrm{s}}$ & $+22^{\circ} 33^{\prime} 39.8^{\prime \prime}$ \\
& $6^{\mathrm{h}} 16^{\mathrm{m}} 41.2^{\mathrm{s}}$ & $+22^{\circ} 33^{\prime} 39.8^{\prime \prime}$ \\
\hline & $6^{\mathrm{h}} 16^{\mathrm{m}} 47.1^{\mathrm{s}}$ & $+22^{\circ} 34^{\prime} 30.7^{\prime \prime}$ \\
& $6^{\mathrm{h}} 16^{\mathrm{m}} 40.2^{\mathrm{s}}$ & $+22^{\circ} 34^{\prime} 30.7^{\prime \prime}$ \\
& $6^{\mathrm{h}} 16^{\mathrm{m}} 40.2^{\mathrm{s}}$ & $+22^{\circ} 32^{\prime} 32.4^{\prime \prime}$ \\
& $6^{\mathrm{h}} 16^{\mathrm{m}} 33.8^{\mathrm{s}}$ & $+22^{\circ} 32^{\prime} 32.4^{\prime \prime}$ \\
& $6^{\mathrm{h}} 16^{\mathrm{m}} 33.8^{\mathrm{s}}$ & $+22^{\circ} 31^{\prime} 30.7^{\prime \prime}$ \\
& $6^{\mathrm{h}} 16^{\mathrm{m}} 31.9^{\mathrm{s}}$ & $+22^{\circ} 31^{\prime} 30.7^{\prime \prime}$ \\
& $6^{\mathrm{h}} 16^{\mathrm{m}} 31.9^{\mathrm{s}}$ & $+22^{\circ} 29^{\prime} 36.6^{\prime \prime}$ \\
& $6^{\mathrm{h}} 16^{\mathrm{m}} 44.7^{\mathrm{s}}$ & $+22^{\circ} 29^{\prime} 36.6^{\prime \prime}$ \\
& $6^{\mathrm{h}} 16^{\mathrm{m}} 44.7^{\mathrm{s}}$ & $+22^{\circ} 31^{\prime} 06.4^{\prime \prime}$ \\
$6^{\mathrm{h}} 16^{\mathrm{m}} 46.2^{\mathrm{s}}$ & $+22^{\circ} 31^{\prime} 06.4^{\prime \prime}$ \\
& $6^{\mathrm{h}} 16^{\mathrm{m}} 46.2^{\mathrm{s}}$ & $+22^{\circ} 32^{\prime} 21.3^{\prime \prime}$ \\
& $6^{\mathrm{h}} 16^{\mathrm{m}} 47.1^{\mathrm{s}}$ & $+22^{\circ} 32^{\prime} 21.3^{\prime \prime}$ \\
\hline & & \\
& &
\end{tabular}

\section{Appendix B: Thermometry of absorption lines}

Considering a transition between an upper level $u$ and a lower level $l$, the extinction coefficient $\kappa_{v}$ is given by

$\kappa_{v}=\frac{c^{2}}{8 \pi v_{\mathrm{ul}}^{2}} A_{\mathrm{ul}} n_{1} \frac{g_{\mathrm{u}}}{g_{\mathrm{l}}}\left[1-\exp \left(\frac{h v_{\mathrm{ul}}}{k T_{\mathrm{ex}}}\right)\right] \phi(v)$,

where $T_{\mathrm{ex}}$ is the excitation temperature, $\phi(v)$ the profile of the line, $v_{\mathrm{ul}}$ the frequency of the transition, $A_{\mathrm{ul}}$ the Einstein coefficient of spontaneous emission, $n_{1}$ the density of the lower level, and $g_{\mathrm{u}}$ and $g_{1}$ are the statistical weights. If $u \leftrightarrow J+1$ and $l \leftrightarrow J$, then the Einstein coefficient is defined by

$A_{J+1 \rightarrow J}=\frac{64 \pi^{4} \mu^{2}}{3 h c^{3}} \frac{J+1}{2 J+3} v_{J}^{3}$

where $\mu$ is the dipole moment of the considered molecule. Thus, by using this definition together with the definition of the statistical weights we get

$\kappa_{v}^{J \rightarrow J+1}=\frac{8 \pi^{3} \mu^{2}}{3 h c} \frac{J+1}{2 J+1} v_{J} n_{J}\left[1-\exp \left(-\frac{h v_{J}}{k T_{\mathrm{ex}}}\right)\right]$.

If we introduce the partition function we get a relation linking $n_{J}$ to the total density $n$ written as

$n_{J}=n \frac{g_{J}}{Q\left(T_{\mathrm{ex}}\right)} \exp \left(-\frac{E_{J}}{k T_{\mathrm{ex}}}\right)$ where $E_{J}$ and $E_{J+1}$ are the level energies. Therefore,

$$
\begin{aligned}
\kappa_{v}^{J \rightarrow J+1}= & \frac{8 \pi^{3} \mu^{2}}{3 h c} v_{J} n \frac{J+1}{Q\left(T_{\mathrm{ex}}\right)} \exp \left(-\frac{E_{J}}{k T_{\mathrm{ex}}}\right) \\
& \times\left[1-\exp \left(\frac{E_{J}-E_{J+1}}{k T_{\mathrm{ex}}}\right)\right] \phi(v) .
\end{aligned}
$$

Thus the total column density $N$ can be obtained by computing the following integral over the line of sight:

$$
\begin{aligned}
N & =\int \mathrm{d} s \int n \phi(v) \mathrm{d} v \\
& =\int \mathrm{d} s \int \frac{\kappa_{v}^{J \rightarrow J+1} \frac{3 h c}{8 \pi^{3} \mu^{2}} \frac{1}{v_{J}} \frac{Q\left(T_{\mathrm{ex}}\right)}{J+1} \exp \left(\frac{E_{J}}{k T_{\mathrm{ex}}}\right)}{\left[1-\exp \left(\frac{E_{J}-E_{J+1}}{k T_{\mathrm{ex}}}\right)\right]} \mathrm{d} v .
\end{aligned}
$$

Using the definition of the equivalent width $W=\int \tau \mathrm{d} v$ and the variable $\mathrm{d} v=v_{J} \mathrm{~d} v / c$, we finally get the relation between column density and equivalent width for a given excitation temperature as follows:

$$
N=\Lambda \frac{Q\left(T_{\mathrm{ex}}\right)}{1-\exp \left(\frac{-h v_{i j}}{k T_{\mathrm{ex}}}\right)} W_{i j},
$$

where $\Lambda=3 h / 8 \pi^{3} \mu^{2}, h$ is the Planck constant, and $v_{i j}$ the frequency of the transition. Applied to the transitions $J=1-0$ and $J=2-1$, this relation can be written for two different values of the equivalent width $W$ corresponding to each line. As the total column density is constant in each equation, the ratio of these two relations yields

$1=\frac{1}{2} \exp \left(\frac{h v_{i j}}{k T_{\mathrm{ex}}}\right) \frac{1-\exp \left(\frac{-h v_{i j}}{k T_{\mathrm{ex}}}\right)}{1-\exp \left(\frac{-2 h v_{i j}}{k T_{\mathrm{ex}}}\right)} \frac{W_{1 \rightarrow 2}}{W_{0 \rightarrow 1}}$.

If we define the temperature of the transition $T_{0}=h v_{i j} / k$ and use the variable $u=T_{0} / T_{\mathrm{ex}}$ then we have

$2=\mathrm{e}^{2 u} \frac{1-\mathrm{e}^{-2 u}}{1-\mathrm{e}^{-4 u}} \frac{W_{1 \rightarrow 2}}{W_{0 \rightarrow 1}}=\mathrm{e}^{3 u} \frac{\mathrm{e}^{u}-\mathrm{e}^{-u}}{\mathrm{e}^{2 u}-\mathrm{e}^{-2 u}} \frac{W_{1 \rightarrow 2}}{W_{0 \rightarrow 1}}$

or

$2=\frac{\mathrm{e}^{3 u}}{2 \cosh (u)} \frac{W_{1 \rightarrow 2}}{W_{0 \rightarrow 1}}$

and, finally, we get the following relation, which permits us to determine the temperature from a measure of the ratio of the two equivalent widths:

$F\left(\frac{T_{0}}{T_{\mathrm{ex}}}\right)=\frac{W_{1 \rightarrow 2}}{4 W_{0 \rightarrow 1}}$,

where $F(u)=\mathrm{e}^{-3 u} \cosh (u)$. 


\section{Appendix C: Spatial distribution of YSO candidates}
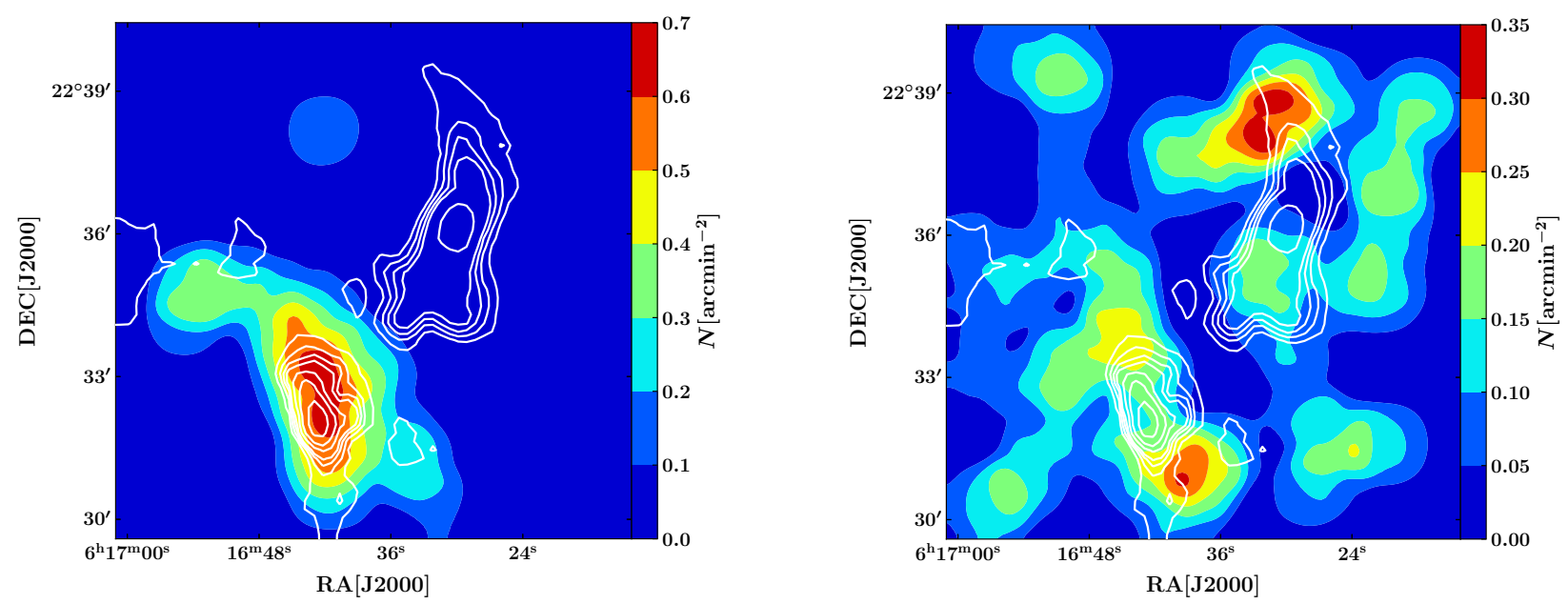

Fig. C.1. Spatial distribution of protostar candidates in our $10^{\prime} \times 10^{\prime}$ field of observations. Left: Class I and Class II candidates detected by WISE. Right: YSO candidates detected by 2 MASS. White contours represent the $\mathrm{CO}(2-1)$ emission in the velocity range $[-7,+3] \mathrm{km} \mathrm{s}^{-1}$.

\section{Appendix D: Complementary channel maps}

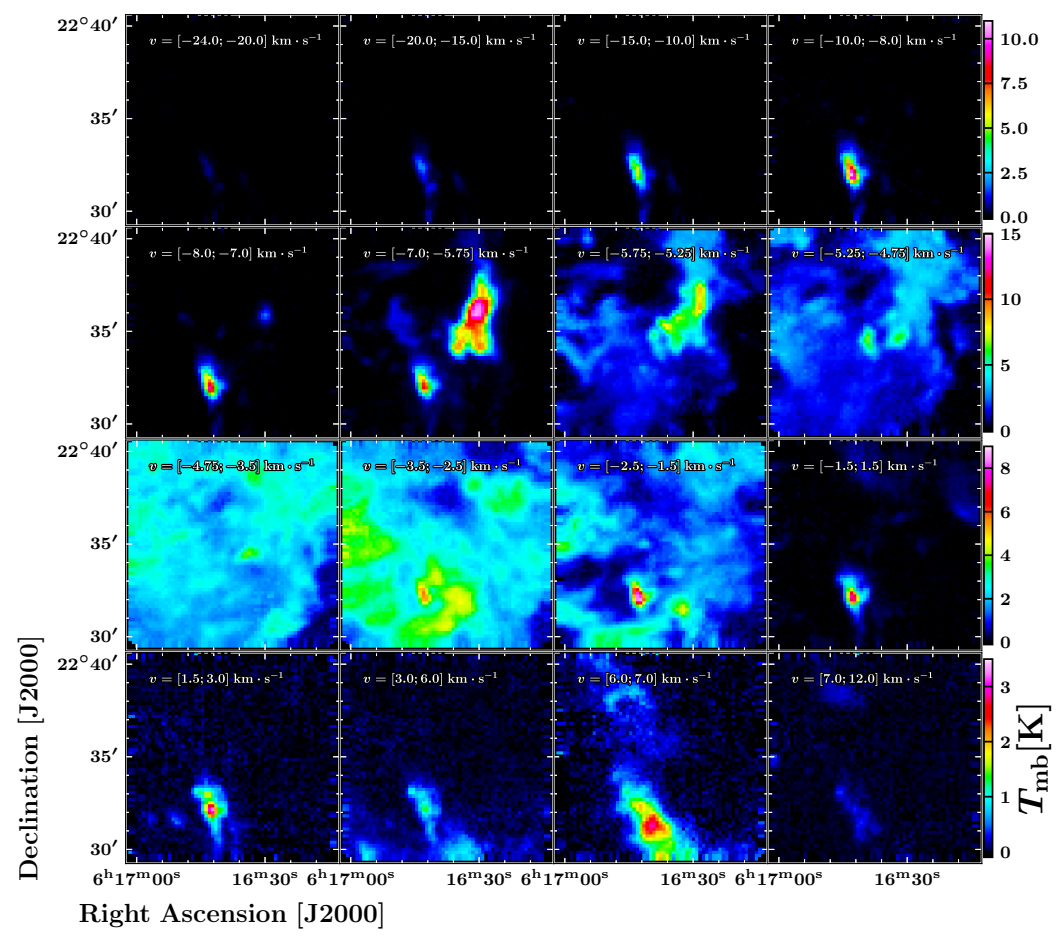

Fig. D.1. Channel map of the ${ }^{12} \mathrm{CO}(1-0)$ observations carried out with the IRAM $30 \mathrm{~m}$ telescope. The $10^{\prime} \times 10^{\prime}$ field is shown in its entirety. Each panel represent the emission integrated over an interval of $0.5 \mathrm{~km} \mathrm{~s}^{-1}$ around a given velocity along the line of sight. Velocities are indicated on the top left corner of each panel. Velocity channels represented on this figure are between $v=-20 \mathrm{~km} \mathrm{~s}^{-1}$ and $v=8 \mathrm{~km} \mathrm{~s}{ }^{-1}$, covering all the spectral features detected toward IC443G. 


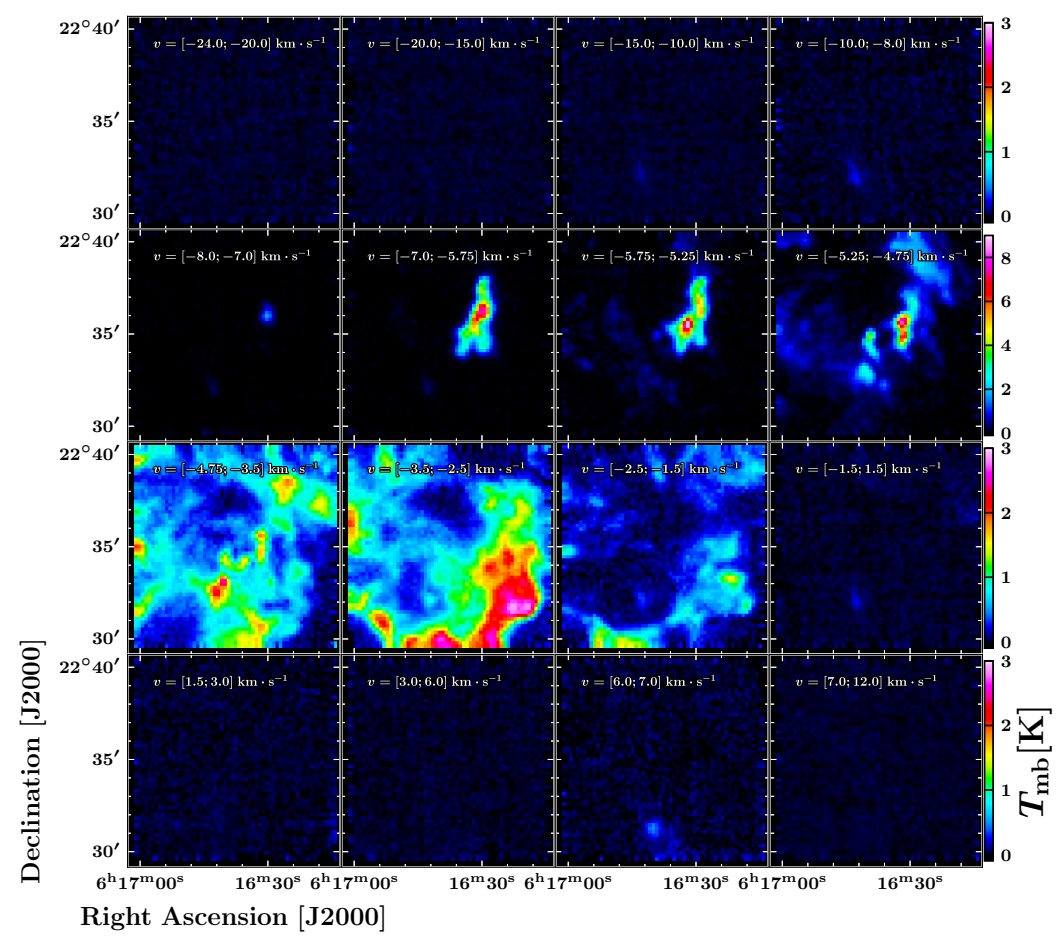

Fig. D.2. Channel map of the ${ }^{13} \mathrm{CO}(1-0)$ observations carried out with the IRAM $30 \mathrm{~m}$ telescope. The $10^{\prime} \times 10^{\prime}$ field is entirely displayed. Each panel represent the emission integrated over an interval of velocity along the line of sight. Velocities are indicated on the top left corner of each panel. Velocity channels represented in this figure are between $v=-24 \mathrm{~km} \mathrm{~s}^{-1}$ and $v=+12 \mathrm{~km} \mathrm{~s}^{-1}$, covering all the spectral features detected toward the extended $\mathrm{G}$ region.

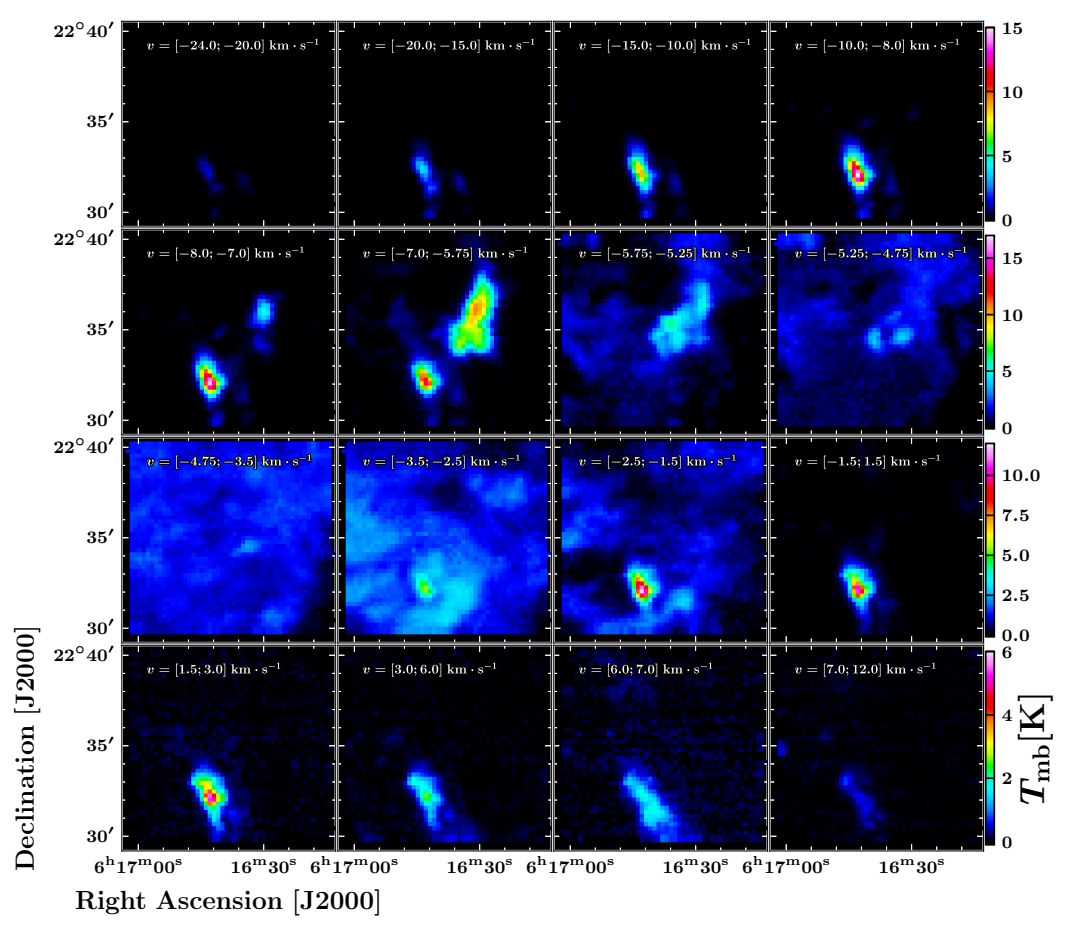

Fig. D.3. Channel map of the ${ }^{12} \mathrm{CO}(2-1)$ observations carried out with the APEX. The $10^{\prime} \times 10^{\prime}$ field is shown in its entirety. Each panel represent the emission integrated over an interval of velocity along the line of sight. Velocities are indicated on the top left corner of each panel. Velocity channels represented in this figure are between $v=-24 \mathrm{~km} \mathrm{~s}^{-1}$ and $v=+12 \mathrm{~km} \mathrm{~s}^{-1}$, covering all the spectral features detected toward the extended G region. 
P. Dell'Ova et al.: Interstellar anatomy of the TeV gamma-ray peak in the IC443 supernova remnant

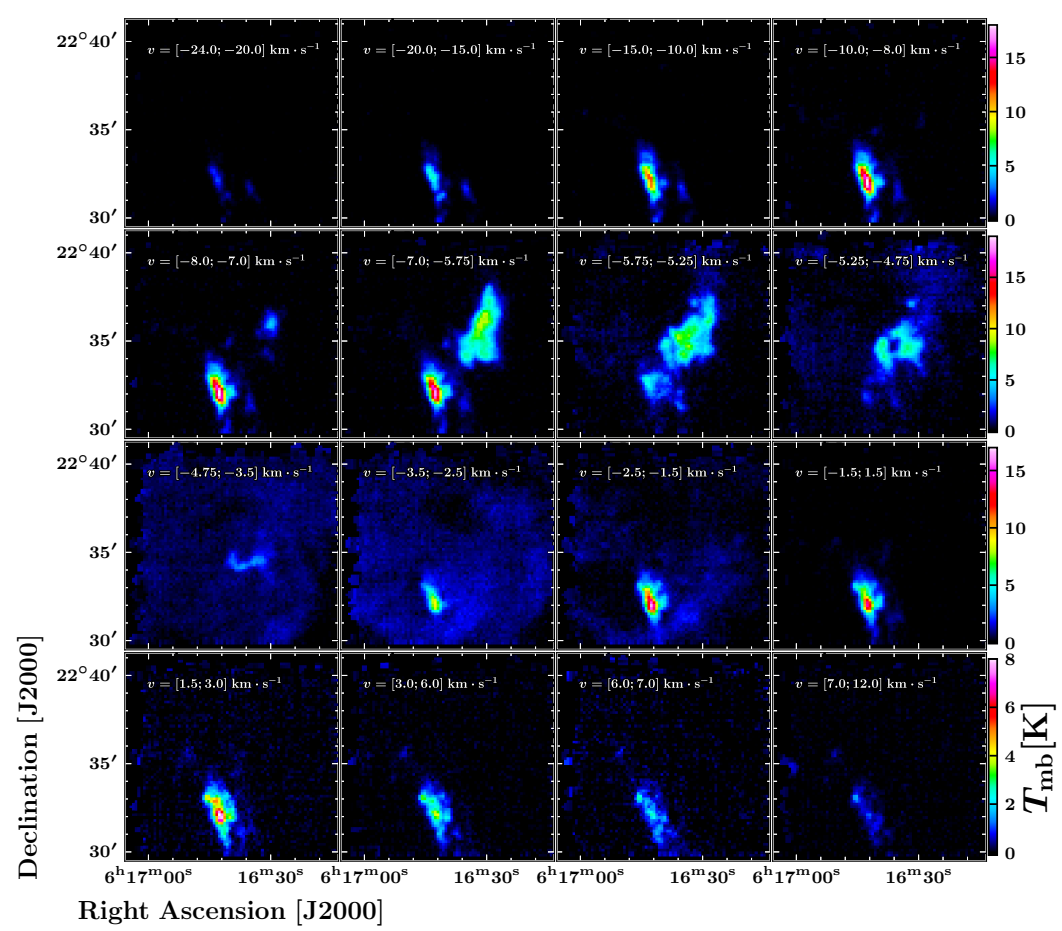

Fig. D.4. Channel map of the ${ }^{12} \mathrm{CO}(3-2)$ observations carried out with APEX. The $10^{\prime} \times 10^{\prime}$ field is shown in its entirety. Each panel represent the emission integrated over an interval of velocity along the line of sight. Velocities are indicated on the top left corner of each panel. Velocity channels represented in this figure are between $v=-24 \mathrm{~km} \mathrm{~s}^{-1}$ and $v=+12 \mathrm{~km} \mathrm{~s}^{-1}$, covering all the spectral features detected toward the extended G region. 


\section{Appendix E: Spectral energy distributions of protostar candidates}

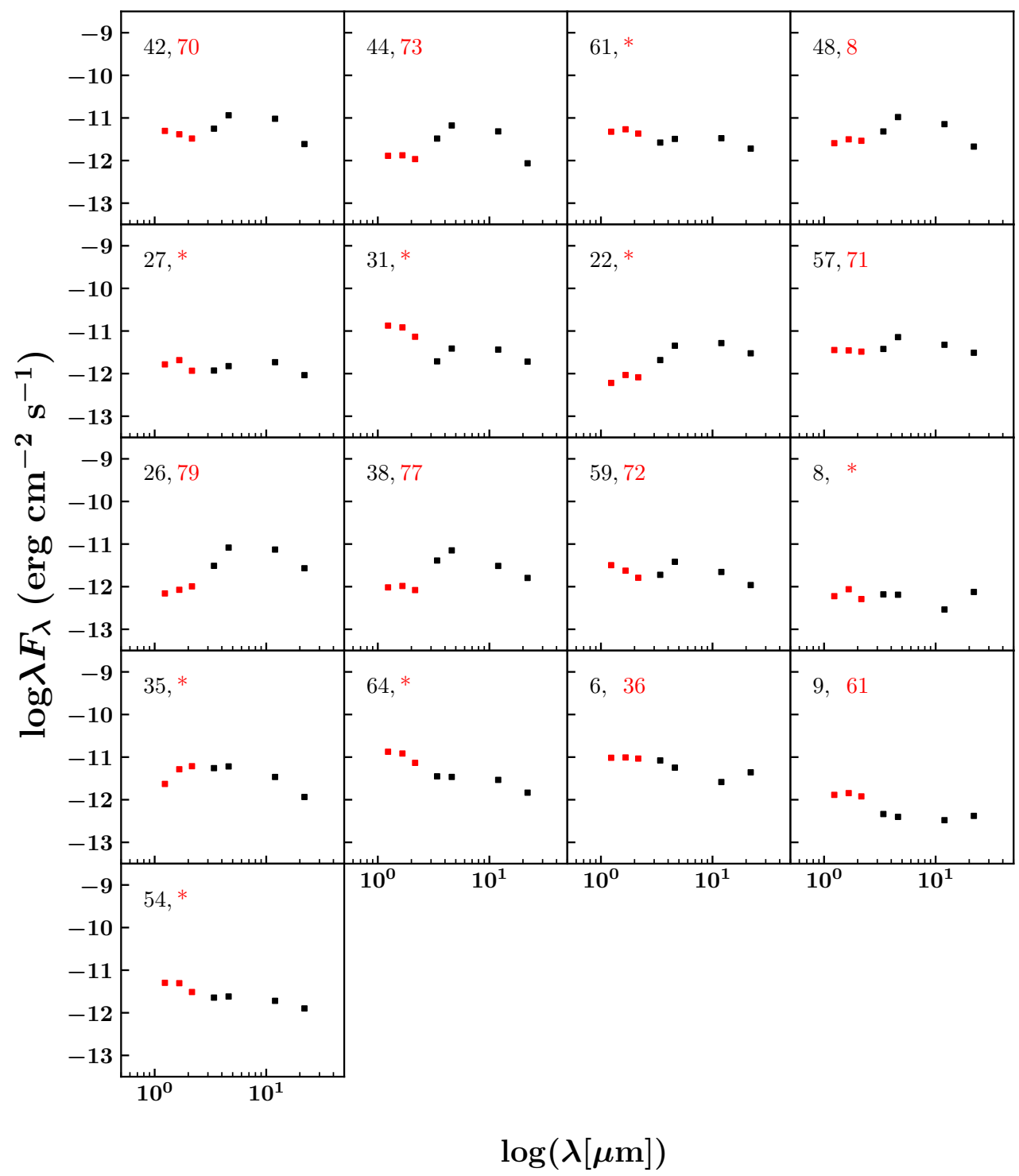

Fig. E.1. Spectral energy distributions of 17 protostar candidates. Their corresponding ID in Tables G.2 and G.1 are indicated in the top left corner of each box when available (marked with an “*” if not corresponding to any point sources identified by a label). Red markers correspond to 2 MASS photometric measures and black markers correspond to WISE measures. 


\section{Appendix F: Observational measure of the optical}

depth
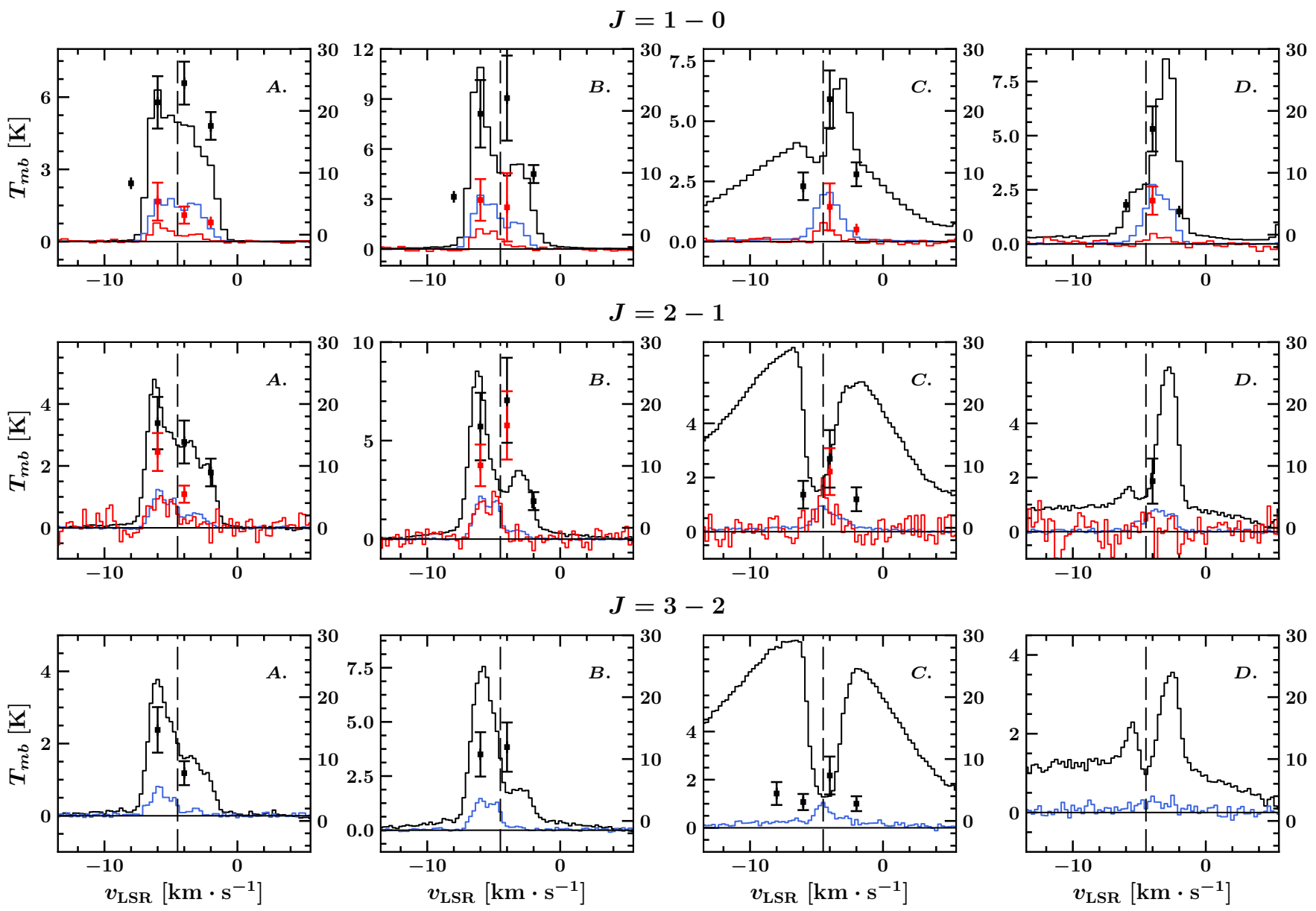

Fig. F.1. Spectra obtained over the region of the four boxes defined in Fig. 6 , for the following lines: ${ }^{12} \mathrm{CO}$ (in black), ${ }^{13} \mathrm{CO}$ (in blue), and $\mathrm{C}^{18} \mathrm{O}$ (in red). First row: (1-0) transition, second row: (2-1) transition, and third row: (3-2) transition for: A, the cloudlet; B, the ring-like structure; C, the shocked clump; and D, the shocked knot. Spectral cubes were resampled to allow a direct comparison between the different spectra. Spatial resolutions of all transitions were modified to the nominal resolution of $\mathrm{C}^{18} \mathrm{O}(2-1), \theta=30.2^{\prime \prime}$. Spectral resolution were set to $0.5 \mathrm{~km} \mathrm{~s}^{-1}$ for the IRAM $30 \mathrm{~m}$ data, and $0.25 \mathrm{~km} \mathrm{~s}^{-1}$ for APEX data. On both panels, the $v_{\mathrm{LSR}}$ of IC443 is indicated with a vertical dashed line (at $-4.5 \mathrm{~km} \mathrm{~s}{ }^{-1}$ ). The black data points with error bars represent the measurements of the optical depth $\tau$ corresponding to each channel of these spectra resampled with a spectral resolution of $2 \mathrm{~km} \mathrm{~s}^{-1}$, using the $\left[{ }^{12} \mathrm{CO}\right] /\left[{ }^{13} \mathrm{CO}\right]$ ratio as a tracer, and the red data points represent the measurements of the optical depth obtained using the $\left[{ }^{12} \mathrm{CO}\right] /\left[\mathrm{C}^{18} \mathrm{O}\right]$ ratio. The adopted values for the isotopic ratios are based on the results of Sect. 4.2.1 and indicated in Table 5. 


\section{Appendix G: WISE and 2MASS based classification of infrared point sources in the extended $\mathrm{G}$ region}

Table G.1. Infrared photometric magnitudes of YSO candidates detected by WISE in the extended G region.

\begin{tabular}{|c|c|c|c|c|c|c|c|c|}
\hline ID & Catalog name & $\begin{array}{c}\mathrm{W} 1(3.4 \mu \mathrm{m}) \\
(\mathrm{mag})\end{array}$ & $\begin{array}{c}\mathrm{W} 2(4.6 \mu \mathrm{m}) \\
(\mathrm{mag})\end{array}$ & $\begin{array}{c}\text { W3 }(12 \mu \mathrm{m}) \\
(\mathrm{mag})\end{array}$ & $\begin{array}{l}\mathrm{W} 4(22 \mu \mathrm{m}) \\
(\mathrm{mag})\end{array}$ & Fig. 13 label & $\alpha$ & FLAG \\
\hline 1 & J061654.93+223403.6 & $17.132 \pm 0.205$ & $16.04 \pm 0.25$ & $11.906 \pm 0.458$ & 8.09 & Class I & 0.99 & 0 \\
\hline 2 & J061655.34+223353.3 & $16.925 \pm 0.206$ & $15.716 \pm 0.225$ & $11.828 \pm 0.52$ & $8.654 \pm 0.508$ & Class I & 0.86 & 0 \\
\hline 3 & J061653.26+223453.7 & $15.348 \pm 0.053$ & $13.717 \pm 0.042$ & $10.316 \pm 0.085$ & 8.234 & Class I & 0.71 & 0 \\
\hline 4 & J061656.75+223829.3 & $15.752 \pm 0.068$ & $14.049 \pm 0.05$ & $10.926 \pm 0.142$ & 8.257 & Class I & 0.53 & 0 \\
\hline 5 & J061617.53+223155.6 & $12.36 \pm 0.025$ & $11.021 \pm 0.021$ & $8.844 \pm 0.03$ & $5.952 \pm 0.049$ & Class I & -0.41 & 0 \\
\hline 6 & J061656.48+223943.2 & $11.276 \pm 0.029$ & $10.733 \pm 0.027$ & $8.612 \pm 0.036$ & $6.027 \pm 0.090$ & Class II & -0.9 & 0 \\
\hline 7 & J061642.63+223825.6 & $15.152 \pm 0.048$ & $14.492 \pm 0.066$ & $12.316 \pm 0.489$ & $8.392 \pm 0.431$ & Class II & -0.79 & 0 \\
\hline 8 & J061617.19+223356.0 & $14.033 \pm 0.031$ & $13.089 \pm 0.032$ & $10.99 \pm 0.197$ & $7.944 \pm 0.219$ & Class II & -0.69 & 0 \\
\hline 9 & J061654.18+223456.6 & $14.42 \pm 0.034$ & $13.622 \pm 0.038$ & $10.85 \pm 0.132$ & $8.582 \pm 0.398$ & Class II & -0.24 & 0 \\
\hline 10 & J061653.02+223444.5 & $15.494 \pm 0.056$ & $14.695 \pm 0.077$ & $11.238 \pm 0.244$ & 8.687 & & 0.29 & 0 \\
\hline 11 & J061639.55+223449.3 & $13.393 \pm 0.042$ & $12.444 \pm 0.029$ & $9.577 \pm 0.371$ & $6.398 \pm 0.141$ & Class II & -0.09 & 0 \\
\hline 12 & J061642.43+223811.8 & $15.687 \pm 0.077$ & $14.321 \pm 0.062$ & $11.897 \pm 0.407$ & $8.415 \pm 0.377$ & Class I & -0.2 & 0 \\
\hline 13 & $\mathrm{~J} 061654.60+223442.0$ & $15.628 \pm 0.056$ & $14.91 \pm 0.094$ & $11.505 \pm 0.258$ & $8.369 \pm 0.310$ & Class II & 0.21 & 0 \\
\hline 14 & J061654.84+223500.4 & $15.476 \pm 0.054$ & $14.818 \pm 0.08$ & $11.6 \pm 0.299$ & 8.268 & Class II & 0.03 & 0 \\
\hline 15 & J061640.86+223753.0 & $15.845 \pm 0.069$ & $14.826 \pm 0.082$ & $11.77 \pm 0.382$ & $8.51 \pm 0.493$ & Class I & 0.1 & 0 \\
\hline 16 & J061638.20+223322.9 & $14.555 \pm 0.049$ & $12.846 \pm 0.035$ & $9.626 \pm 0.113$ & $7.84 \pm 0.366$ & Class I & 0.61 & 1 \\
\hline 17 & J061638.34+223313.0 & $14.453 \pm 0.059$ & $13.053 \pm 0.035$ & $9.533 \pm 0.2$ & $7.877 \pm 0$ & & 0.68 & 1 \\
\hline 18 & J061643.64+223232.1 & $11.158 \pm 0.028$ & $9.394 \pm 0.021$ & $6.363 \pm 0.015$ & $6.11 \pm 0.145$ & & 0.4 & 3 \\
\hline 19 & $\mathrm{~J} 061646.10+223246.9$ & $13.34 \pm 0.028$ & $11.706 \pm 0.023$ & $8.424 \pm 0.029$ & 8.667 & Class I & 0.62 & 3 \\
\hline 20 & J061641.30+223044.8 & $15.073 \pm 0.053$ & $13.683 \pm 0.044$ & $9.385 \pm 0.087$ & 8.617 & Class I & 1.28 & 3 \\
\hline 21 & J061642.50+223142.4 & $11.168 \pm 0.025$ & $9.246 \pm 0.021$ & $6.51 \pm 0.015$ & $6.217 \pm 0.090$ & Class I & 0.35 & 3 \\
\hline 22 & J061634.95+223128.1 & $12.786 \pm 0.036$ & $10.981 \pm 0.024$ & & $6.443 \pm 0.071$ & & 0.59 & 3 \\
\hline 23 & J061639.57+223219.9 & $13.203 \pm 0.045$ & $11.801 \pm 0.037$ & $8.653 \pm 0.13$ & 8.272 & Class I & 0.39 & 5 \\
\hline 24 & J061632.40+223012.2 & $14.098 \pm 0.036$ & $12.352 \pm 0.028$ & $8.965 \pm 0.037$ & $7.88 \pm 0.305$ & Class I & 0.76 & 1 \\
\hline 25 & J061642.44+223206.3 & $11.228 \pm 0.028$ & $9.299 \pm 0.023$ & $6.521 \pm 0.018$ & $6.171 \pm 0.103$ & & 0.39 & 5 \\
\hline 26 & J061643.86+223340.7 & $12.358 \pm 0.048$ & $10.323 \pm 0.027$ & $7.473 \pm 0.053$ & 6.553 & Clas & 0.5 & 3 \\
\hline 27 & J061642.75+223108.9 & $13.4 \pm 0.026$ & $12.176 \pm 0.026$ & $8.98 \pm 0.035$ & $7.724 \pm 0.211$ & & 0.32 & 3 \\
\hline 28 & J061641.63+223111.0 & $12.829 \pm 0.027$ & $11.003 \pm 0.023$ & $8.096 \pm 0.029$ & $7.607 \pm 0.289$ & Class I & 0.43 & 5 \\
\hline 29 & J061635.24+223521.1 & $14.723 \pm 0.057$ & $13.606 \pm 0.042$ & $9.478 \pm 0.202$ & $7.199 \pm 0.156$ & Class I & 1.0 & 1 \\
\hline 30 & J061639.81+223201.5 & $12.88 \pm 0.031$ & $10.896 \pm 0.023$ & $8.251 \pm 0.0$ & 8.371 & $\mathrm{Cla}$ & 0.31 & 3 \\
\hline 31 & J061633.23+223103.7 & $12.862 \pm 0.027$ & $11.146 \pm 0.023$ & $8.242 \pm 0.043$ & $6.932 \pm 0.098$ & $\mathrm{Cla}$ & 0.37 & 3 \\
\hline 32 & J061635.54+223108.5 & $13.757 \pm 0.03$ & $12.12 \pm 0.026$ & $8.638 \pm 0.039$ & $7.456 \pm 0.162$ & & 0.78 & 3 \\
\hline 33 & J061642.91+223215.9 & $11.159 \pm 0.024$ & $9.3 \pm 0.02$ & $6.33 \pm 0.014$ & $5.976 \pm 0.084$ & Class I & 0.5 & 3 \\
\hline 34 & J061639.38+223234.8 & $14.358 \pm 0.046$ & $13.055 \pm 0.038$ & $9.669 \pm 0.23$ & 7.606 & Class I & 0.52 & 3 \\
\hline 35 & J061640.86+223310.8 & $11.727 \pm 0.023$ & $10.662 \pm 0.02$ & $8.315 \pm 0.036$ & $7.473 \pm 0.244$ & Class II & -0.43 & 5 \\
\hline 36 & J061647.39+223406.4 & $13.909 \pm 0.03$ & 13.397 & $8 \pm 0.212$ & 7.292 & Class II & -0.66 & 3 \\
\hline 37 & J061642.00+223339.7 & $10.74 \pm 0.024$ & $10.143 \pm 0.02$ & $8.244 \pm 0.085$ & $6.939 \pm 0.193$ & Class II & -1 . & 3 \\
\hline 38 & J061645.55+223335.1 & $12.046 \pm 0.025$ & $10.487 \pm 0.021$ & $8.433 \pm 0.032$ & 7.121 & Class I & -0.39 & 5 \\
\hline 39 & J061635.51+223239.1 & $14.932 \pm 0.044$ & $13.438 \pm 0.035$ & $11.349 \pm 0.366$ & 8.485 & Class I & -0.4 & 3 \\
\hline 40 & J061644.88+223352.6 & $12.305 \pm 0.039$ & & & & & & 3 \\
\hline 41 & J061642.28+223241.6 & $11.956 \pm 0.034$ & $10.024 \pm 0.023$ & $7.769 \pm 0.105$ & $6.777 \pm 0.128$ & Class I & -0.02 & 3 \\
\hline 42 & $\mathrm{~J} 061643.22+223302.2$ & $11.709 \pm 0.027$ & $9.96 \pm 0.021$ & $7.198 \pm 0.018$ & $6.666 \pm 0.254$ & Class I & 0.27 & 3 \\
\hline 43 & J061647.94+223431.0 & $13.987 \pm 0.032$ & $12.795 \pm 0.028$ & $9.7 \pm 0.078$ & 7.89 & Class I & 0.23 & 3 \\
\hline 44 & J061640.55+223121.5 & $12.291 \pm 0.025$ & $10.557 \pm 0.023$ & $7.939 \pm 0.026$ & $7.795 \pm 0.259$ & Class I & 0.15 & 5 \\
\hline 45 & J061630.48+223009.3 & $14.817 \pm 0.041$ & $13.375 \pm 0.042$ & $10.875 \pm 0.17$ & 8.639 & Class I & -0.1 & 1 \\
\hline 46 & J061643.19+223038.8 & $15.019 \pm 0.043$ & $13.721 \pm 0.04$ & $11.043 \pm 0.184$ & 8.752 & Class I & -0.04 & 1 \\
\hline 47 & J061650.62+223515.5 & $13.783 \pm 0.032$ & $11.77 \pm 0.024$ & $9.615 \pm 0.062$ & $8.559 \pm 0.490$ & Class I & -0.06 & 1 \\
\hline 48 & J061641.59+223132.8 & $11.874 \pm 0.029$ & $10.064 \pm 0.023$ & $7.515 \pm 0.022$ & $6.815 \pm 0.146$ & Class I & 0.14 & 3 \\
\hline 49 & J061640.18+223149.4 & $12.626 \pm 0.031$ & $10.845 \pm 0.029$ & $8.508 \pm 0.088$ & 7.898 & Class I & -0.04 & 5 \\
\hline 50 & J061639.32+223320.1 & $14.693 \pm 0.061$ & $12.991 \pm 0.035$ & $10.655 \pm 0.284$ & $7.322 \pm 0.192$ & Class I & -0.09 & 1 \\
\hline 51 & J061636.38+223308.9 & $13.925 \pm 0.038$ & $12.355 \pm 0.027$ & $10.027 \pm 0.396$ & 7.67 & & -0.17 & 1 \\
\hline 52 & J061642.01+223418.9 & $14.356 \pm 0.057$ & $12.241 \pm 0.026$ & $9.799 \pm 0.462$ & $7.823 \pm 0.482$ & Class I & 0.23 & 3 \\
\hline
\end{tabular}


Table G.1. continued.

\begin{tabular}{|c|c|c|c|c|c|c|c|}
\hline 53 & J061639.74+223041.6 & $14.382 \pm 0.036$ & $12.701 \pm 0.034$ & $9.991 \pm 0.347$ & 8.641 & Class I & 0.2 \\
\hline 54 & J061646.36+223424.7 & $12.69 \pm 0.027$ & $11.661 \pm 0.024$ & $8.949 \pm 0.045$ & $7.379 \pm 0.251$ & Class II & -0.16 \\
\hline 55 & J061645.01+223259.5 & $11.93 \pm 0.024$ & $10.383 \pm 0.02$ & $7.495 \pm 0.019$ & $7.311 \pm 0.138$ & Class I & 0.26 \\
\hline 56 & J061643.87+223250.1 & $11.07 \pm 0.024$ & $9.276 \pm 0.02$ & $6.732 \pm 0.016$ & $6.358 \pm 0.194$ & Class I & 0.13 \\
\hline 57 & J061635.06+223135.9 & $12.134 \pm 0.026$ & $10.475 \pm 0.023$ & $7.958 \pm 0.025$ & $6.41 \pm 0.073$ & Class I & 0.03 \\
\hline 58 & $\mathrm{~J} 061647.01+223500.8$ & $14.047 \pm 0.035$ & $11.907 \pm 0.024$ & $9.892 \pm 0.103$ & 7.989 & Class I & -0.1 \\
\hline 59 & J061644.84+223442.6 & $12.885 \pm 0.029$ & $11.157 \pm 0.026$ & $8.788 \pm 0.035$ & $7.539 \pm 0.240$ & Class I & -0.05 \\
\hline 60 & J061641.36+223012.4 & $13.6 \pm 0.037$ & $11.791 \pm 0.027$ & $9.673 \pm 0.122$ & 7.951 & Class I & -0.2 \\
\hline 61 & J061643.18+223129.7 & $12.525 \pm 0.022$ & $11.347 \pm 0.023$ & $8.34 \pm 0.024$ & $6.933 \pm 0.111$ & Class I & 0.15 \\
\hline 62 & J061650.94+223454.3 & $14.969 \pm 0.049$ & $12.959 \pm 0.032$ & $10.635 \pm 0.144$ & 8.476 & Class I & 0.07 \\
\hline 63 & J061644.45+223331.1 & $12.396 \pm 0.035$ & $10.456 \pm 0.022$ & $7.952 \pm 0.027$ & 7.341 & Class I & 0.18 \\
\hline 64 & J061633.49+223110.4 & $12.208 \pm 0.024$ & $11.279 \pm 0.027$ & $8.486 \pm 0.039$ & $7.219 \pm 0.144$ & Class II & -0.16 \\
\hline 65 & J061642.76+223402.6 & $12.266 \pm 0.028$ & $10.421 \pm 0.021$ & $7.897 \pm 0.071$ & $7.022 \pm 0.162$ & Class I & 0.14 \\
\hline
\end{tabular}

Table G.2. Infrared photometric magnitudes of YSO candidates detected by 2MASS in the extended G region.

\begin{tabular}{|c|c|c|c|c|c|c|c|}
\hline ID & $\begin{array}{c}\text { RA } \\
(\mathrm{J} 2000)\end{array}$ & $\begin{array}{c}\text { Dec } \\
(\mathrm{J} 2000)\end{array}$ & $\begin{array}{c}J(1.25 \mu \mathrm{m}) \\
(\mathrm{mag})\end{array}$ & $\begin{array}{c}H(1.65 \mu \mathrm{m}) \\
(\mathrm{mag})\end{array}$ & $\begin{array}{c}K(2.17 \mu \mathrm{m}) \\
(\mathrm{mag})\end{array}$ & Fig. 13 label & FLAG \\
\hline 1 & $6^{\mathrm{h}} 16^{\mathrm{m}} 21.16^{\mathrm{s}}$ & $22^{\mathrm{d}} 37^{\mathrm{m}} 25.37^{\mathrm{s}}$ & $16.073 \pm 0.078$ & $15.449 \pm 0.108$ & $14.932 \pm 0.093$ & YSO & 0 \\
\hline 2 & $6^{\mathrm{h}} 16^{\mathrm{m}} 21.35^{\mathrm{s}}$ & $22^{\mathrm{d}} 34^{\mathrm{m}} 32.27^{\mathrm{s}}$ & $15.3 \pm 0.048$ & $14.92 \pm 0.087$ & $14.708 \pm 0.075$ & YSO & 0 \\
\hline 3 & $6^{\mathrm{h}} 16^{\mathrm{m}} 32.01^{\mathrm{s}}$ & $22^{\mathrm{d}} 33^{\mathrm{m}} 59.85^{\mathrm{s}}$ & $16.83 \pm 0.140$ & $15.981 \pm 0.182$ & $15.234 \pm 0.120$ & YSO & 0 \\
\hline 4 & $6^{\mathrm{h}} 16^{\mathrm{m}} 23.77^{\mathrm{s}}$ & $22^{\mathrm{d}} 34^{\mathrm{m}} 59.89^{\mathrm{s}}$ & $15.138 \pm 0.055$ & $14.519 \pm 0.064$ & $14.094 \pm 0.046$ & YSO & 0 \\
\hline 5 & $6^{\mathrm{h}} 16^{\mathrm{m}} 19.73^{\mathrm{s}}$ & $22^{\mathrm{d}} 36^{\mathrm{m}} 17.92^{\mathrm{s}}$ & $15.439 \pm 0.074$ & $14.997 \pm 0.094$ & $14.576 \pm 0.070$ & YSO & 0 \\
\hline 6 & $6^{\mathrm{h}} 16^{\mathrm{m}} 21.42^{\mathrm{s}}$ & $22^{\mathrm{d}} 31^{\mathrm{m}} 33.23^{\mathrm{s}}$ & $15.4 \pm 0.052$ & $14.815 \pm 0.069$ & $14.347 \pm 0.067$ & YSO & 0 \\
\hline 7 & $6^{\mathrm{h}} 16^{\mathrm{m}} 20.25^{\mathrm{s}}$ & $22^{\mathrm{d}} 31^{\mathrm{m}} 39.30^{\mathrm{s}}$ & $16.75 \pm 0.150$ & $16.051 \pm 0.192$ & $15.478 \pm 0.136$ & YSO & 0 \\
\hline 8 & $6^{\mathrm{h}} 16^{\mathrm{m}} 54.05^{\mathrm{s}}$ & $22^{\mathrm{d}} 30^{\mathrm{m}} 37.07^{\mathrm{s}}$ & $16.742 \pm 0.165$ & $16.013 \pm 0.188$ & $15.451 \pm 0.145$ & YSO & 0 \\
\hline 9 & $6^{\mathrm{h}} 16^{\mathrm{m}} 39.00^{\mathrm{s}}$ & $22^{\mathrm{d}} 30^{\mathrm{m}} 00.26^{\mathrm{s}}$ & $16.22 \pm 0.109$ & $15.587 \pm 0.144$ & $15.226 \pm 0.123$ & YSO & 0 \\
\hline 10 & $6^{\mathrm{h}} 16^{\mathrm{m}} 41.73^{\mathrm{s}}$ & $22^{\mathrm{d}} 30^{\mathrm{m}} 21.36^{\mathrm{s}}$ & $15.163 \pm 0.049$ & $14.663 \pm 0.067$ & $14.226 \pm 0.054$ & YSO & 0 \\
\hline 11 & $6^{\mathrm{h}} 16^{\mathrm{m}} 45.23^{\mathrm{s}}$ & $22^{\mathrm{d}} 31^{\mathrm{m}} 03.71^{\mathrm{s}}$ & $16.753 \pm 0.170$ & $16.174 \pm 0.222$ & $15.604 \pm 0.168$ & YSO & 0 \\
\hline 12 & $6^{\mathrm{h}} 16^{\mathrm{m}} 37.41^{\mathrm{s}}$ & $22^{\mathrm{d}} 30^{\mathrm{m}} 45.04^{\mathrm{s}}$ & $16.45 \pm 0.120$ & $16.011 \pm 0.188$ & $15.756 \pm 0.189$ & YSO & 0 \\
\hline 13 & $6^{\mathrm{h}} 16^{\mathrm{m}} 28.65^{\mathrm{s}}$ & $22^{\mathrm{d}} 30^{\mathrm{m}} 29.60^{\mathrm{s}}$ & $15.273 \pm 0.061$ & $14.65 \pm 0.090$ & $14.086 \pm 0.059$ & YSO & 0 \\
\hline 14 & $6^{\mathrm{h}} 16^{\mathrm{m}} 25.18^{\mathrm{s}}$ & $22^{\mathrm{d}} 31^{\mathrm{m}} 48.32^{\mathrm{s}}$ & $13.487 \pm 0.027$ & $13.011 \pm 0.038$ & $12.658 \pm 0.025$ & YSO & 0 \\
\hline 15 & $6^{\mathrm{h}} 16^{\mathrm{m}} 25.96^{\mathrm{s}}$ & $22^{\mathrm{d}} 30^{\mathrm{m}} 57.38^{\mathrm{s}}$ & $15.913 \pm 0.072$ & $15.315 \pm 0.105$ & $14.878 \pm 0.107$ & YSO & 0 \\
\hline 16 & $6^{\mathrm{h}} 16^{\mathrm{m}} 26.89^{\mathrm{s}}$ & $22^{\mathrm{d}} 31^{\mathrm{m}} 46.11^{\mathrm{s}}$ & $15.278 \pm 0.044$ & $14.761 \pm 0.075$ & $14.47 \pm 0.068$ & YSO & 0 \\
\hline 17 & $6^{\mathrm{h}} 16^{\mathrm{m}} 37.10^{\mathrm{s}}$ & $22^{\mathrm{d}} 31^{\mathrm{m}} 17.74^{\mathrm{s}}$ & $16.608 \pm 0.148$ & $15.972 \pm 0.194$ & $15.335 \pm 0.134$ & YSO & 0 \\
\hline 18 & $6^{\mathrm{h}} 16^{\mathrm{m}} 18.27^{\mathrm{s}}$ & $22^{\mathrm{d}} 38^{\mathrm{m}} 49.88^{\mathrm{s}}$ & $14.252 \pm 0.034$ & $13.7 \pm 0.034$ & $13.392 \pm 0.030$ & YSO & 0 \\
\hline 19 & $6^{\mathrm{h}} 16^{\mathrm{m}} 17.72^{\mathrm{s}}$ & $22^{\mathrm{d}} 38^{\mathrm{m}} 57.65^{\mathrm{s}}$ & $16.62 \pm 0.132$ & $16.029 \pm 0.190$ & $15.464 \pm 0.145$ & YSO & 0 \\
\hline 20 & $6^{\mathrm{h}} 16^{\mathrm{m}} 27.76^{\mathrm{s}}$ & $22^{\mathrm{d}} 39^{\mathrm{m}} 04.81^{\mathrm{s}}$ & $16.348 \pm 0.106$ & $15.627 \pm 0.162$ & $15.161 \pm 0.121$ & YSO & 0 \\
\hline 21 & $6^{\mathrm{h}} 16^{\mathrm{m}} 39.20^{\mathrm{s}}$ & $22^{\mathrm{d}} 36^{\mathrm{m}} 57.77^{\mathrm{s}}$ & $17.06 \pm 0.183$ & $16.282 \pm 0.235$ & $15.662 \pm 0.170$ & YSO & 0 \\
\hline 22 & $6^{\mathrm{h}} 16^{\mathrm{m}} 38.84^{\mathrm{s}}$ & $22^{\mathrm{d}} 37^{\mathrm{m}} 50.51^{\mathrm{s}}$ & $13.85 \pm 0.027$ & $13.508 \pm 0.039$ & $13.307 \pm 0.031$ & YSO & 0 \\
\hline 23 & $6^{\mathrm{h}} 16^{\mathrm{m}} 34.85^{\mathrm{s}}$ & $22^{\mathrm{d}} 37^{\mathrm{m}} 45.31^{\mathrm{s}}$ & $13.714 \pm 0.031$ & $13.268 \pm 0.038$ & $12.954 \pm 0.027$ & YSO & 0 \\
\hline 24 & $6^{\mathrm{h}} 16^{\mathrm{m}} 32.52^{\mathrm{s}}$ & $22^{\mathrm{d}} 38^{\mathrm{m}} 19.35^{\mathrm{s}}$ & $14.745 \pm 0.036$ & $14.165 \pm 0.055$ & $13.813 \pm 0.039$ & YSO & 0 \\
\hline 25 & $6^{\mathrm{h}} 16^{\mathrm{m}} 30.79^{\mathrm{s}}$ & $22^{\mathrm{d}} 38^{\mathrm{m}} 27.47^{\mathrm{s}}$ & $14.896 \pm 0.036$ & $14.202 \pm 0.034$ & $13.762 \pm 0.039$ & YSO & 0 \\
\hline 26 & $6^{\mathrm{h}} 16^{\mathrm{m}} 30.19^{\mathrm{s}}$ & $22^{\mathrm{d}} 38^{\mathrm{m}} 29.64^{\mathrm{s}}$ & $15.996 \pm 0.079$ & $15.408 \pm 0.114$ & $14.843 \pm 0.087$ & YSO & 0 \\
\hline 27 & $6^{\mathrm{h}} 16^{\mathrm{m}} 33.70^{\mathrm{s}}$ & $22^{\mathrm{d}} 37^{\mathrm{m}} 27.99^{\mathrm{s}}$ & $14.368 \pm 0.032$ & $13.821 \pm 0.047$ & $13.487 \pm 0.031$ & YSO & 0 \\
\hline 28 & $6^{\mathrm{h}} 16^{\mathrm{m}} 33.83^{\mathrm{s}}$ & $22^{\mathrm{d}} 38^{\mathrm{m}} 06.52^{\mathrm{s}}$ & $15.865 \pm 0.072$ & $15.212 \pm 0.087$ & $14.83 \pm 0.078$ & YSO & 0 \\
\hline 29 & $6^{\mathrm{h}} 16^{\mathrm{m}} 56.77^{\mathrm{s}}$ & $22^{\mathrm{d}} 30^{\mathrm{m}} 15.22^{\mathrm{s}}$ & $15.112 \pm 0.042$ & $14.755 \pm 0.070$ & $14.461 \pm 0.060$ & YSO & 0 \\
\hline 30 & $6^{\mathrm{h}} 16^{\mathrm{m}} 53.13^{\mathrm{s}}$ & $22^{\mathrm{d}} 31^{\mathrm{m}} 03.76^{\mathrm{s}}$ & $15.879 \pm 0.082$ & $15.605 \pm 0.149$ & $15.273 \pm 0.131$ & YSO & 0 \\
\hline 31 & $6^{\mathrm{h}} 16^{\mathrm{m}} 57.99^{\mathrm{s}}$ & $22^{\mathrm{d}} 33^{\mathrm{m}} 04.98^{\mathrm{s}}$ & $14.739 \pm 0.037$ & $14.366 \pm 0.057$ & $14.057 \pm 0.048$ & YSO & 0 \\
\hline 32 & $6^{\mathrm{h}} 16^{\mathrm{m}} 48.47^{\mathrm{s}}$ & $22^{\mathrm{d}} 31^{\mathrm{m}} 57.35^{\mathrm{s}}$ & $11.835 \pm 0.023$ & $11.741 \pm 0.030$ & $11.69 \pm 0.023$ & YSO & 0 \\
\hline 33 & $6^{\mathrm{h}} 16^{\mathrm{m}} 49.15^{\mathrm{s}}$ & $22^{\mathrm{d}} 33^{\mathrm{m}} 37.91^{\mathrm{s}}$ & $16.26 \pm 0.100$ & $15.794 \pm 0.152$ & $15.355 \pm 0.129$ & YSO & 0 \\
\hline 34 & $6^{\mathrm{h}} 16^{\mathrm{m}} 51.04^{\mathrm{s}}$ & $22^{\mathrm{d}} 33^{\mathrm{m}} 16.69^{\mathrm{s}}$ & $16.806 \pm 0.178$ & $16.093 \pm 0.223$ & $15.637 \pm 0.169$ & YSO & 0 \\
\hline 35 & $6^{\mathrm{h}} 16^{\mathrm{m}} 52.65^{\mathrm{s}}$ & $22^{\mathrm{d}} 32^{\mathrm{m}} 10.02^{\mathrm{s}}$ & $15.536 \pm 0.056$ & $14.916 \pm 0.077$ & $14.473 \pm 0.063$ & YSO & 0 \\
\hline 36 & $6^{\mathrm{h}} 16^{\mathrm{m}} 56.50^{\mathrm{s}}$ & $22^{\mathrm{d}} 39^{\mathrm{m}} 43.29^{\mathrm{s}}$ & $14.008 \pm 0.038$ & $13.187 \pm 0.041$ & $12.502 \pm 0.028$ & YSO & 0 \\
\hline 37 & $6^{\mathrm{h}} 16^{\mathrm{m}} 49.04^{\mathrm{s}}$ & $22^{\mathrm{d}} 39^{\mathrm{m}} 47.80^{\mathrm{s}}$ & $14.418 \pm 0.033$ & $14.196 \pm 0.048$ & $13.853 \pm 0.044$ & YSO & 0 \\
\hline
\end{tabular}


Table G.2. continued.

\begin{tabular}{|c|c|c|c|c|c|c|c|}
\hline ID & $\begin{array}{c}\text { RA } \\
(\mathrm{J} 2000)\end{array}$ & $\begin{array}{c}\text { Dec } \\
(\mathrm{J} 2000)\end{array}$ & $\begin{array}{c}J(1.25 \mu \mathrm{m}) \\
(\mathrm{mag})\end{array}$ & $\begin{array}{c}H(1.65 \mu \mathrm{m}) \\
(\mathrm{mag})\end{array}$ & $\begin{array}{c}K(2.17 \mu \mathrm{m}) \\
(\mathrm{mag})\end{array}$ & Fig. 13 label & FLAG \\
\hline 38 & $6^{\mathrm{h}} 16^{\mathrm{m}} 49.76^{\mathrm{s}}$ & $22^{\mathrm{d}} 39^{\mathrm{m}} 12.79^{\mathrm{s}}$ & $15.869 \pm 0.078$ & $15.481 \pm 0.123$ & $15.182 \pm 0.117$ & YSO & 0 \\
\hline 39 & $6^{\mathrm{h}} 16^{\mathrm{m}} 58.80^{\mathrm{s}}$ & $22^{\mathrm{d}} 35^{\mathrm{m}} 58.44^{\mathrm{s}}$ & $11.262 \pm 0.022$ & $10.979 \pm 0.030$ & $10.796 \pm 0.021$ & YSO & 0 \\
\hline 40 & $6^{\mathrm{h}} 16^{\mathrm{m}} 52.79^{\mathrm{s}}$ & $22^{\mathrm{d}} 39^{\mathrm{m}} 39.43^{\mathrm{s}}$ & $16.178 \pm 0.103$ & $16.14 \pm 0.228$ & $15.496 \pm 0.164$ & YSO & 0 \\
\hline 41 & $6^{\mathrm{h}} 16^{\mathrm{m}} 51.69^{\mathrm{s}}$ & $22^{\mathrm{d}} 35^{\mathrm{m}} 37.42^{\mathrm{s}}$ & $15.277 \pm 0.052$ & $14.827 \pm 0.074$ & $14.372 \pm 0.081$ & YSO & 0 \\
\hline 42 & $6^{\mathrm{h}} 16^{\mathrm{m}} 40.28^{\mathrm{s}}$ & $22^{\mathrm{d}} 38^{\mathrm{m}} 17.54^{\mathrm{s}}$ & $15.355 \pm 0.056$ & $14.797 \pm 0.082$ & $14.487 \pm 0.068$ & YSO & 0 \\
\hline 43 & $6^{\mathrm{h}} 16^{\mathrm{m}} 41.82^{\mathrm{s}}$ & $22^{\mathrm{d}} 37^{\mathrm{m}} 30.09^{\mathrm{s}}$ & $16.673 \pm 0.158$ & $16.052 \pm 0.215$ & $15.654 \pm 0.167$ & YSO & 0 \\
\hline 44 & $6^{\mathrm{h}} 16^{\mathrm{m}} 44.95^{\mathrm{s}}$ & $22^{\mathrm{d}} 35^{\mathrm{m}} 49.96^{\mathrm{s}}$ & $15.919 \pm 0.081$ & $15.28 \pm 0.100$ & $14.832 \pm 0.086$ & YSO & 0 \\
\hline 45 & $6^{\mathrm{h}} 16^{\mathrm{m}} 50.66^{\mathrm{s}}$ & $22^{\mathrm{d}} 37^{\mathrm{m}} 20.93^{\mathrm{s}}$ & $14.637 \pm 0.040$ & $14.328 \pm 0.057$ & $14.092 \pm 0.055$ & YSO & 0 \\
\hline 46 & $6^{\mathrm{h}} 16^{\mathrm{m}} 18.14^{\mathrm{s}}$ & $22^{\mathrm{d}} 38^{\mathrm{m}} 18.74^{\mathrm{s}}$ & $16.236 \pm 0.102$ & $15.326 \pm 0.118$ & $14.713 \pm 0.069$ & CTTS & 0 \\
\hline 47 & $6^{\mathrm{h}} 16^{\mathrm{m}} 20.71^{\mathrm{s}}$ & $22^{\mathrm{d}} 36^{\mathrm{m}} 44.49^{\mathrm{s}}$ & $16.83 \pm 0.163$ & $16.054 \pm 0.190$ & $15.544 \pm 0.151$ & CTTS & 0 \\
\hline 48 & $6^{\mathrm{h}} 16^{\mathrm{m}} 31.07^{\mathrm{s}}$ & $22^{\mathrm{d}} 35^{\mathrm{m}} 20.79^{\mathrm{s}}$ & $17.069 \pm 0.203$ & $15.948 \pm 0.175$ & $15.21 \pm 0.128$ & CTTS & 0 \\
\hline 49 & $6^{\mathrm{h}} 16^{\mathrm{m}} 28.25^{\mathrm{s}}$ & $22^{\mathrm{d}} 33^{\mathrm{m}} 54.94^{\mathrm{s}}$ & $15.992 \pm 0.094$ & $15.117 \pm 0.090$ & $14.573 \pm 0.072$ & CTTS & 0 \\
\hline 50 & $6^{\mathrm{h}} 16^{\mathrm{m}} 32.20^{\mathrm{s}}$ & $22^{\mathrm{d}} 34^{\mathrm{m}} 52.98^{\mathrm{s}}$ & $17.311 \pm 0.228$ & $15.919 \pm 0.169$ & $15.075 \pm 0.112$ & CTTS & 0 \\
\hline 51 & $6^{\mathrm{h}} 16^{\mathrm{m}} 25.32^{\mathrm{s}}$ & $22^{\mathrm{d}} 35^{\mathrm{m}} 29.40^{\mathrm{s}}$ & $16.402 \pm 0.098$ & $15.57 \pm 0.136$ & $14.91 \pm 0.093$ & CTTS & 0 \\
\hline 52 & $6^{\mathrm{h}} 16^{\mathrm{m}} 41.39^{\mathrm{s}}$ & $22^{\mathrm{d}} 32^{\mathrm{m}} 57.89^{\mathrm{s}}$ & $16.827 \pm 0.180$ & $15.657 \pm 0.153$ & $14.944 \pm 0.111$ & CTTS & 0 \\
\hline 53 & $6^{\mathrm{h}} 16^{\mathrm{m}} 28.97^{\mathrm{s}}$ & $22^{\mathrm{d}} 32^{\mathrm{m}} 39.40^{\mathrm{s}}$ & $15.688 \pm 0.056$ & $14.903 \pm 0.064$ & $14.459 \pm 0.064$ & CTTS & 0 \\
\hline 54 & $6^{\mathrm{h}} 16^{\mathrm{m}} 25.67^{\mathrm{s}}$ & $22^{\mathrm{d}} 39^{\mathrm{m}} 06.09^{\mathrm{s}}$ & $17.201 \pm 0.219$ & $16.343 \pm 0.262$ & $15.773 \pm 0.198$ & CTTS & 0 \\
\hline 55 & $6^{\mathrm{h}} 16^{\mathrm{m}} 34.49^{\mathrm{s}}$ & $22^{\mathrm{d}} 35^{\mathrm{m}} 22.21^{\mathrm{s}}$ & $16.21 \pm 0.084$ & $15.149 \pm 0.084$ & $14.537 \pm 0.063$ & CTTS & 0 \\
\hline 56 & $6^{\mathrm{h}} 16^{\mathrm{m}} 30.92^{\mathrm{s}}$ & $22^{\mathrm{d}} 36^{\mathrm{m}} 01.72^{\mathrm{s}}$ & $16.564 \pm 0.128$ & $15.298 \pm 0.110$ & $14.436 \pm 0.062$ & CTTS & 0 \\
\hline 57 & $6^{\mathrm{h}} 16^{\mathrm{m}} 30.75^{\mathrm{s}}$ & $22^{\mathrm{d}} 39^{\mathrm{m}} 28.69^{\mathrm{s}}$ & $16.132 \pm 0.079$ & $15.328 \pm 0.099$ & $14.737 \pm 0.077$ & CTTS & 0 \\
\hline 58 & $6^{\mathrm{h}} 16^{\mathrm{m}} 32.27^{\mathrm{s}}$ & $22^{\mathrm{d}} 39^{\mathrm{m}} 32.10^{\mathrm{s}}$ & $16.987 \pm 0.166$ & $15.842 \pm 0.152$ & $15.084 \pm 0.107$ & CTTS & 0 \\
\hline 59 & $6^{\mathrm{h}} 16^{\mathrm{m}} 50.15^{\mathrm{s}}$ & $22^{\mathrm{d}} 33^{\mathrm{m}} 04.16^{\mathrm{s}}$ & $16.416 \pm 0.122$ & $15.609 \pm 0.138$ & $15.122 \pm 0.105$ & CTTS & 0 \\
\hline 60 & $6^{\mathrm{h}} 16^{\mathrm{m}} 49.96^{\mathrm{s}}$ & $22^{\mathrm{d}} 38^{\mathrm{m}} 43.88^{\mathrm{s}}$ & $16.521 \pm 0.136$ & $15.645 \pm 0.143$ & $15.08 \pm 0.113$ & CTTS & 0 \\
\hline 61 & $6^{\mathrm{h}} 16^{\mathrm{m}} 54.18^{\mathrm{s}}$ & $22^{\mathrm{d}} 34^{\mathrm{m}} 56.75^{\mathrm{s}}$ & $16.184 \pm 0.098$ & $15.279 \pm 0.107$ & $14.72 \pm 0.076$ & CTTS & 0 \\
\hline 62 & $6^{\mathrm{h}} 16^{\mathrm{m}} 48.71^{\mathrm{s}}$ & $22^{\mathrm{d}} 36^{\mathrm{m}} 02.72^{\mathrm{s}}$ & $16.682 \pm 0.152$ & $15.854 \pm 0.171$ & $15.249 \pm 0.126$ & CTTS & 0 \\
\hline 63 & $6^{\mathrm{h}} 16^{\mathrm{m}} 20.50^{\mathrm{s}}$ & $22^{\mathrm{d}} 37^{\mathrm{m}} 06.78^{\mathrm{s}}$ & $15.847 \pm 0.074$ & $15.298 \pm 0.102$ & $14.635 \pm 0.083$ & $\mathrm{HAeBe}$ & 0 \\
\hline 64 & $6^{\mathrm{h}} 16^{\mathrm{m}} 20.35^{\mathrm{s}}$ & $22^{\mathrm{d}} 35^{\mathrm{m}} 04.23^{\mathrm{s}}$ & $15.896 \pm 0.073$ & $15.477 \pm 0.110$ & $14.917 \pm 0.100$ & HAeBe & 0 \\
\hline 65 & $6^{\mathrm{h}} 16^{\mathrm{m}} 37.47^{\mathrm{s}}$ & $22^{\mathrm{d}} 30^{\mathrm{m}} 53.17^{\mathrm{s}}$ & $16.922 \pm 0.187$ & $16.207 \pm 0.243$ & $15.484 \pm 0.150$ & $\mathrm{HAeBe}$ & 0 \\
\hline 66 & $6^{\mathrm{h}} 16^{\mathrm{m}} 25.69^{\mathrm{s}}$ & $22^{\mathrm{d}} 38^{\mathrm{m}} 08.44^{\mathrm{s}}$ & $16.448 \pm 0.140$ & $15.86 \pm 0.161$ & $15.218 \pm 0.132$ & $\mathrm{HAeBe}$ & 0 \\
\hline 67 & $6^{\mathrm{h}} 16^{\mathrm{m}} 31.69^{\mathrm{s}}$ & $22^{\mathrm{d}} 38^{\mathrm{m}} 17.72^{\mathrm{s}}$ & $16.433 \pm 0.108$ & $16.133 \pm 0.215$ & $15.392 \pm 0.138$ & $\mathrm{HAeBe}$ & 0 \\
\hline 68 & $6^{\mathrm{h}} 16^{\mathrm{m}} 57.74^{\mathrm{s}}$ & $22^{\mathrm{d}} 30^{\mathrm{m}} 24.12^{\mathrm{s}}$ & $16.068 \pm 0.092$ & $15.669 \pm 0.144$ & $15.076 \pm 0.101$ & HAeBe & 0 \\
\hline 69 & $6^{\mathrm{h}} 16^{\mathrm{m}} 57.50^{\mathrm{s}}$ & $22^{\mathrm{d}} 34^{\mathrm{m}} 34.24^{\mathrm{s}}$ & $16.757 \pm 0.159$ & $16.112 \pm 0.217$ & $15.46 \pm 0.157$ & $\mathrm{HAeBe}$ & 0 \\
\hline 70 & $6^{\mathrm{h}} 16^{\mathrm{m}} 43.12^{\mathrm{s}}$ & $22^{\mathrm{d}} 33^{\mathrm{m}} 01.40^{\mathrm{s}}$ & $14.73 \pm 0.042$ & $14.126 \pm 0.053$ & $13.625 \pm 0.086$ & YSO & 2 \\
\hline 71 & $6^{\mathrm{h}} 16^{\mathrm{m}} 35.10^{\mathrm{s}}$ & $22^{\mathrm{d}} 31^{\mathrm{m}} 33.98^{\mathrm{s}}$ & $15.086 \pm 0.048$ & $14.306 \pm 0.051$ & $13.628 \pm 0.056$ & YSO & 2 \\
\hline 72 & $6^{\mathrm{h}} 16^{\mathrm{m}} 44.82^{\mathrm{s}}$ & $22^{\mathrm{d}} 34^{\mathrm{m}} 42.82^{\mathrm{s}}$ & $15.207 \pm 0.049$ & $14.728 \pm 0.067$ & $14.395 \pm 0.061$ & YSO & 2 \\
\hline 73 & $6^{\mathrm{h}} 16^{\mathrm{m}} 40.21^{\mathrm{s}}$ & $22^{\mathrm{d}} 31^{\mathrm{m}} 15.34^{\mathrm{s}}$ & $16.192 \pm 0.104$ & $15.358 \pm 0.119$ & $14.831 \pm 0.119$ & CTTS & 2 \\
\hline 74 & $6^{\mathrm{h}} 16^{\mathrm{m}} 41.46^{\mathrm{s}}$ & $22^{\mathrm{d}} 31^{\mathrm{m}} 28.03^{\mathrm{s}}$ & $15.451 \pm 0.061$ & $14.421 \pm 0.058$ & $13.757 \pm 0.061$ & CTTS & 2 \\
\hline 75 & $6^{\mathrm{h}} 16^{\mathrm{m}} 43.47^{\mathrm{s}}$ & $22^{\mathrm{d}} 31^{\mathrm{m}} 55.73^{\mathrm{s}}$ & $16.435 \pm 0.121$ & $15.65 \pm 0.139$ & $15.131 \pm 0.135$ & CTTS & 2 \\
\hline 76 & $6^{\mathrm{h}} 16^{\mathrm{m}} 44.87^{\mathrm{s}}$ & $22^{\mathrm{d}} 34^{\mathrm{m}} 10.32^{\mathrm{s}}$ & $14.743 \pm 0.046$ & $14.039 \pm 0.057$ & $13.62 \pm 0.072$ & CTTS & 1 \\
\hline 77 & $6^{\mathrm{h}} 16^{\mathrm{m}} 45.52^{\mathrm{s}}$ & $22^{\mathrm{d}} 33^{\mathrm{m}} 42.53^{\mathrm{s}}$ & $16.511 \pm 0.132$ & $15.626 \pm 0.144$ & $15.116 \pm 0.162$ & CTTS & 2 \\
\hline 78 & $6^{\mathrm{h}} 16^{\mathrm{m}} 45.50^{\mathrm{s}}$ & $22^{\mathrm{d}} 34^{\mathrm{m}} 58.63^{\mathrm{s}}$ & $16.432 \pm 0.130$ & $15.51 \pm 0.140$ & $14.975 \pm 0.135$ & CTTS & 1 \\
\hline 79 & $6^{\mathrm{h}} 16^{\mathrm{m}} 43.49^{\mathrm{s}}$ & $22^{\mathrm{d}} 33^{\mathrm{m}} 37.44^{\mathrm{s}}$ & $16.867 \pm 0.189$ & $15.85 \pm 0.178$ & $14.899 \pm 0.148$ & HAeBe & 2 \\
\hline
\end{tabular}

